\title{
Spectral Sparsification and Regret Minimization Beyond Matrix Multiplicative Updates*
}

\author{
Zeyuan Allen-Zhu \\ zeyuan@csail.mit.edu \\ MIT CSAIL
}

\author{
Zhenyu Liao \\ zhenyul@bu.edu \\ Boston University
}

\begin{tabular}{|l|}
\hline Lorenzo Orecchia \\
\hline orecchia@bu.edu \\
Boston University
\end{tabular}

March 09, 2015

\begin{abstract}
In this paper, we provide a novel construction of the linear-sized spectral sparsifiers of Batson, Spielman and Srivastava [BSS14]. While previous constructions required $\Omega\left(n^{4}\right)$ running time [BSS14. Zou12], our sparsification routine can be implemented in almost-quadratic running time $O\left(n^{2+\varepsilon}\right)$.

The fundamental conceptual novelty of our work is the leveraging of a strong connection between sparsification and a regret minimization problem over density matrices. This connection was known to provide an interpretation of the randomized sparsifiers of Spielman and Srivastava [SS11] via the application of matrix multiplicative weight updates (MWU) [CHS11. Vis14]. In this paper, we explain how matrix MWU naturally arises as an instance of the Follow-theRegularized-Leader framework and generalize this approach to yield a larger class of updates. This new class allows us to accelerate the construction of linear-sized spectral sparsifiers, and give novel insights on the motivation behind Batson, Spielman and Srivastava [BSS14].
\end{abstract}

\footnotetext{
${ }^{*}$ Part of this work was performed when the third author was an instructor at MIT Math, and when all the authors were visiting the Simons Institute at Berkeley.
} 


\section{Introduction}

A powerful tool to handle large-scaled graphs is to compress them by reducing their sizes, while preserving properties of interest such as the size of cuts $[\mathrm{BK} 96$. BK02] or the routability of certain flows [CLLM10]. This sparsification procedures also play an important role as fundamental primitives behind many fast graph algorithms [KLOS14. PS14]. In this paper, we consider the strong notion of spectral sparsifier put forward by Spielman and Teng [ST04. ST11]: $G^{\prime}$ is $(1+\varepsilon)$-spectral approximate to $G$ if $G^{\prime}$ is a subgraph of $G$ with possibly reweighted edges, and for every $x \in \mathbb{R}^{n}$,

$$
x^{T} L_{G} x \leq x^{T} L_{G^{\prime}} x \leq(1+\varepsilon) x^{T} L_{G} x \quad \text { or equivalently } \quad L_{G} \preceq L_{G^{\prime}} \preceq(1+\varepsilon) L_{G} ，
$$

where $L_{G}$ and $L_{G^{\prime}}$ are respectively the graph Laplacian matrices of $G$ and $G^{\prime}$.

The algorithm of Spielman and Srivastava SS11] constructs $(1+\varepsilon)$-spectral sparsifiers with $O\left(n \log n / \varepsilon^{2}\right)$ edges in nearly linear time by randomly sampling edges proportionally to their effective resistance. In a seminal paper, Batson, Spielman and Srivastava [BSS14] give $(1+\varepsilon)$-spectral sparsifiers with $O\left(n / \varepsilon^{2}\right)$ edges, but their construction and subsequent algorithm by [Zou12] require $O\left(m n^{3} / \varepsilon^{2}\right)$ and $O\left(m n^{2} / \varepsilon^{2}+n^{4} / \varepsilon^{4}\right)$ time respectively. We shall refer to their analysis and algorithm the BSS for short. The main contribution of this paper is to give an improved construction of linear-sized spectral sparsifiers that runs in almost-quadratic time.

Theorem 1. For any even integer $q \geq 2$ and any $\varepsilon \in\left(0, \frac{1}{4 \sqrt{q}}\right)$, there is an algorithm that, for any weighted undirected graph $G$ with $n$ vertices and $m$ edges, with probability at least $1-n^{-\Omega(1)}$, constructs a $(1+\varepsilon)$-spectral sparsifier $G^{\prime}$ that has at most $O\left(\sqrt{q} n / \varepsilon^{2}\right)$ edges in time $\widetilde{O}\left(m n^{1+1 / q} / \varepsilon^{5}\right)$.

Since $q$ can be chosen as a large constant and the graph can be preprocessed to reduce the number of edges to $m=O(n \log n)$, the above running time is almost quadratic in terms of $n$.

Graph sparsification is a special case of sparsifying sums of rank-1 PSD matrices (see [BSS14] and Appendix B). Our algorithm for Theorem 1 also applies to this more general problem with an almost cubic running time, which is stil an improvement over the previous quartic running time.

Theorem 2. For any even integer $q \geq 2$ and any $\varepsilon \in\left(0, \frac{1}{4 \sqrt{q}}\right)$, there is an algorithm that, for any decomposition $I=\sum_{i=1}^{m} v_{i} v_{i}^{T} \in \mathbb{R}^{n \times n}$ of rank-1 matrices, with probability at least $1-n^{-\Omega(1)}$, constructs scalars $s_{i} \geq 0$ with $\left|\left\{i: s_{i}>0\right\}\right| \leq O\left(\sqrt{q} n / \varepsilon^{2}\right)$ that satisfies $I \preceq \sum_{i=1}^{m} s_{i} v_{i} v_{i}^{T} \preceq(1+\varepsilon) I$ in time $\widetilde{O}\left(n^{3+1 / q} / \varepsilon^{5}+m n / \varepsilon^{4}\right)$.

The fundamental conceptual novelty of our work is the establishment of a deep connection between graph or matrix sparsifications and a regret minimization problem over PSD matrices (see Section 1.1). This relation was known [CHS11. Vis14] for the randomized sparsifiers of Spielman and Srivastava [SS11], for which the underlying matrix concentration bound can be easily recovered as an application of the matrix version of Multiplicative Weight Updates (MWU) [AK07. Ore11], a standard online learning algorithm. However, it was not clear how this interpretation could be extended to BSS, despite a clear analogy was also noted by de Carli Silva, Harvey and Sato (see CHS11. Section 8]). Both the MWU and the BSS rely on potential function arguments, where the potential is essentially a robust version to capture of the maximum and minimum graph eigenvalues. In this paper, we provide the missing piece of this interpretation: we consider a generalization of MWU to a larger class of updates, and show that the BSS can be recovered as an instance of this class. Beyond our faster implementation of sparsification, we believe that this interpretation is of independent interest and may be useful in other areas in which the argument of BSS has found application [Nao12].

We focus on updates coming from the follow-the-regularized-leader (FTRL) framework. The choice of regularizer in this framework fully determines the update strategy and the corresponding potential function. See for example the recent survey by Hazan Haz12]. The standard MWU 
argument can be recovered as an instance of FTRL, where the regularizer is chosen to be the entropy function. In contrast, we choose a different class of regularizers consisting of all $\ell_{1-1 / q}$ semi-norms for $q \geq 2$, and provide corresponding regret bounds in Section 3. In Section 4 and Section 5. we show that the choice $q=2$ recovers an algorithm which is somewhat similar to BSS, and produces linear-sized spectral sparsifiers. This algorithm can be implemented to run in a $O\left(m n^{3 / 2}\right)$ time. Finally, in Section 6, we consider regularizers corresponding to large, constant $q>2$, which yield very different algorithms from BSS with almost quadratic running time.

\subsection{Regret Minimization}

In this subsection, we discuss our contribution on the problem of regret minimization in online linear optimization [Haz12]. Our technical results apply to the more general case of online PSD linear optimization over the set of density matrices, but our key contributions are described more concisely in the scalar case.

Let $\Delta_{n}=\left\{x \in \mathbb{R}^{n}: x \geq 0 \wedge \mathbb{1}^{T} x=1\right\}$ be the unit simplex in $\mathbb{R}^{n}$, and we call a vector in $\Delta_{n}$ an action. A player is going to play $T$ actions $x_{0}, \ldots, x_{T-1} \in \Delta_{n}$ in a row; only after playing $x_{k}$, the player observes a feedback vector $f_{k} \in \mathbb{R}^{n}$, which may depend on $x_{k}$, and suffers the linear loss $\left\langle f_{k}, x_{k}\right\rangle$. The regret minimization problem asks us to device a strategy for the player that minimizes the regret, i.e., difference between the total loss suffered by the player and the loss suffered by the $a$ posteriori best fixed action $u \in \Delta_{n}$ :

$$
\text { minimize } \max _{u \in \Delta_{n}} R(u), \quad \text { where } R(u) \stackrel{\text { def }}{=} \sum_{k=0}^{T-1}\left\langle f_{k}, x_{k}-u\right\rangle \text {. }
$$

A well-known strategy for this problem is to update $x_{k}$ in a multiplicative fashion: for each coordinate $i \in[n]$, define $x_{k+1, i}$ to be proportional to $x_{k, i} \cdot \exp ^{-\alpha \cdot f_{k, i}}$ for some parameter $\alpha>0$. This strategy is known as the multiplicative weight update. Its classical analysis [AHK12] implies

$$
\forall u \in \Delta_{n}, \quad R(u)=\sum_{k=0}^{T-1}\left\langle f_{k}, x_{k}-u\right\rangle \leq \frac{\alpha}{2} \sum_{k=0}^{T-1}\left\|f_{k}\right\|_{\infty}^{2}+\frac{\log n}{\alpha} .
$$

The first term on the righthand side contributes a regret of $\left\|f_{k}\right\|_{\infty}^{2}$ that is paid at every iteration, and we call it the width term. The second term is a fixed start-up cost corresponding to 'how long it takes the update to explore the whole $\Delta_{n}$ ', and we call it the diameter term. If for all iterations $k,\left\|f_{k}\right\|_{\infty}$ is upper bounded by $\rho$, known as the width of the problem, the trade-off between the width and diameter terms can be be optimized by the choice of $\alpha>0$ to show that the total regret is at most $O(\rho \sqrt{T \log n})$.

Optimization Interpretation. We take an optimization perspective to describe MWU and its generalizations by characterizing our strategies as instances of the follow-the-regularized-leader and mirror descent frameworks. Let $w(\cdot)$ be a strongly convex function over the simplex, known as the regularizer. The follow-the-regularized-leader strategy with parameter $\alpha>0$ can be described as a trade-off between minimizing the loss incurred so far and the value of the regularizer.

$$
\text { FTRL: } \quad x_{k+1}=\underset{z \in \Delta_{n}}{\arg \min }\left\{w(z)+\alpha \sum_{j=0}^{k}\left\langle f_{j}, z\right\rangle\right\} .
$$

Similarly, the mirror-descent strategy optimizes a trade-off

$$
\text { MirrorDescent: } \quad \text { start with } x_{0}=\left(\frac{1}{n}, \ldots, \frac{1}{n}\right) ; \quad x_{k+1} \leftarrow \underset{z \in \Delta_{n}}{\arg \min }\left\{V_{x_{k}}(z)+\alpha\left\langle f_{k}, z\right\rangle\right\},
$$

where $V_{x}(y) \stackrel{\text { def }}{=} w(y)-w(x)-\langle\nabla w(x), y-x\rangle$ is the induced Bregman divergence. Under mild assumptions (which are satisfied in this paper, see Appendix A), it is easy to check that MirrorDescent is equivalent to FTRL. We will therefore interchangeably use MirrorDescent and FTRL in the rest of the paper, because FTRL gives the cleaner description for the updates, while MirrorDescent 
provides a simpler analysis. The MWU strategy is an instance of the two equivalent strategies above, with the choice of regularizer $w(x) \stackrel{\text { def }}{=} \sum_{i} x_{i} \log x_{i}-x_{i}$, i.e. the (negative) entropy function.

Previous Work. The MWU is a simple but extremely powerful algorithmic tool that has been repeatedly discovered in theory of computation, machine learning, optimization, and game theory (see for instance the survey [AHK12] and the book [CL06]). Since MWU has found numerous important applications in semidefinite programming [AK07. AHK05], constraint satisfaction problem [Ste10], maximum flow [CKM $\left.{ }^{+} 11\right]$, sparsest cut [She09], balanced separator [OSV12], small set expansion $\left.\mathrm{BFK}^{+} 11\right]$, traveling salesman problem $\left[\mathrm{AGM}^{+} 10\right]$, zero-sum games [DDK11], and fractional packing problems [GK07]. The analysis of follow-the-regularized-leader can be found in the surveys [Haz12. Sha07], while that of the mirror descent appears in the the book [BN13].

Beyond MWU. Historically, MWU has been extended at least from three orthogonal directions. In this paper, we pursue all these three directions simultaneously (see our summary in Table 1.)

1. From vector to matrix. Instead of studying actions $x$ in the forms of $n$-dimensional probability distributions, one can study density matrices $X$ in $\Delta_{n \times n}$, the set of PSD matrices whose trace equals to one. This is a generalization from a set of "experts" corresponding to $\left\{\mathbf{e}_{1}, \ldots, \mathbf{e}_{n}\right\}$ to all combinations of the form $\sum_{i=1}^{n} t_{i} \mathbf{e}_{i}$ where $t$ is on the $n$-dimensional unit sphere $\mathbb{S}^{n-1}$. Accordingly, each loss vector $f_{k}$ can be generalized to a symmetric matrix $F_{k} \in \mathbb{R}^{n \times n}$, so the loss of any density matrix $X$ becomes $F_{k} \bullet X=\operatorname{Tr}\left(F_{k} X\right)$. (If $X=v v^{T}$ is of rank one, then $F_{k} \bullet X=v^{T} F_{k} v$.) Among many applications, the matrix version of MWU has been used in designing algorithms for solving semidefinite programs [AK07] and finding balanced separators [OSV12], and in the proof of QIP = PSPACE [JJUW11].

2. Local norm convergence. The width term $\left\|f_{k}\right\|_{\infty}^{2}$ in the regret upper bound (1.1) can be replaced with $\left\langle\left|f_{k}\right|, x_{k}\right\rangle \cdot\left\|f_{k}\right\|_{\infty}$. (Here, we have used $\left|f_{k}\right|$ to denote coordinate-wise absolute value of $f_{k}$.) This technique is known as the local-norm technique because $\left\langle\left|f_{k}\right|, x_{k}\right\rangle$ is a local way to measure the length of $f_{k}$ with respect to $x_{k}$. Since $\left\langle\left|f_{k}\right|, x_{k}\right\rangle \cdot\left\|f_{k}\right\|_{\infty}$ is never larger than $\left\|f_{k}\right\|_{\infty}^{2}$, as well as $x_{k} \in \Delta_{n}$, this new upper bound can only be smaller than the original. Indeed, this tighter bound has proved useful in the multi-arm bandit problem [AHR12], and in the solution of positive linear programs [AO15]. It also underpins the negative-width technique of [AHK12].

3. Change of regularizer. If one replaces the entropy regularizer with the $\ell_{1-1 / q}$-regularizer $w(x)=-\frac{q}{q-1} \sum_{i=1}^{n} x_{i}^{1-1 / q}$ for any $q \geq 2$, the corresponding update rule changes

$$
\text { from } x_{k+1, i}=\exp ^{-\sum_{j=0}^{k} \alpha f_{j, i}+c} \text { to } x_{k+1, i}=\left(\sum_{j=0}^{k} \alpha f_{j, i}+c\right)^{-q} \text {, }
$$

where in both cases $c$ is the unique constant that ensures $x_{k+1} \in \Delta_{n}$. The FTRL framework is very powerful as the choice of regularizer $w(x)$ completely determines both the form and the analysis of the update strategy. Ultimately, different regularizers achieve different trade-offs between the width and diameter terms in Equation (1.1). For instance, the $\ell_{1 / 2}$-regularizer yields the following regret bound

$$
\forall u \in \Delta_{n}, \quad R(u) \leq O(\alpha) \cdot \sum_{k=0}^{T-1}\left\langle\left|f_{k}\right|, x_{k}\right\rangle \cdot \max _{i \in[n]}\left|f_{k, i} \sqrt{x_{k, i}}\right|+\frac{2 \sqrt{n}}{\alpha} .
$$

The diameter term is now $2 \sqrt{n}$, much worse than $\log n$ in the entropy case in (1.1). However, since (the local norm version of) the width term goes from $\left\langle\left|f_{k}\right|, x_{k}\right\rangle \cdot\left\|f_{k}\right\|_{\infty}$ to $\left\langle\left|f_{k}\right|, x_{k}\right\rangle$. $\max _{i \in[n]}\left|f_{k, i} \sqrt{x_{k, i}}\right|$, the width term may become smaller.. This is exactly the case in the sparsification case, where the feedback vectors, corresponding to the edges added to the 


\begin{tabular}{|c|c|c|c|}
\hline Paper & Allow Matrix? & Allow Local Norm? & Allow Non-Entropy Regularizer? \\
\hline [PST95. FS95] & no & no & no \\
\hline AHK05, AHK12] & yes & no \\
\hline AHR12, AO15] & no & yes & yes \\
\hline ABL11. BC12] & no & no & no \\
\hline AK07. OSV12] & yes & yes & no \\
\hline$[$ HKS12 & yes & yes & yes \\
\hline this paper $]$ & yes &
\end{tabular}

Table 1: Comparisons among prior results on the regret minimization problem.

sparsifier, may be weighted up by a factor as large as $n$, so that we may have $\left\|f_{k}\right\|_{\infty} \geq n$. In this scenario, the use of a more stongly-convex regularizer, such as $\ell_{1 / 2}$, allows us to measure the width in a more convenient local norm and yields the BSS linear-sized sparsifier(see Figure 1 on page 12 for a visual comparison of different regularizers). We point out that the $\ell_{1-1 / q}$-regularizers have also been used, albeit solely in the scalar case, by the machine learning community to obtain asymptotically optimal strategies for the multi-arm bandit problem [ABL11. BC12].

\subsection{Extensions}

High Rank Sparsification. Our same algorithm of Theorem 1 and 2 also applies to sparsifying sums of PSD matrices, rather than just rank-1 PSD matrices. This recovers the same result of de Carli Silva, Harvey, and Sato [CHS11]. Such an extension has been shown important for problems such as finding hypergraph sparsifiers, finding sparse SDP solutions, and finding sparsifiers on subgraphs. However, as in the rank-1 case, the detailed running time of our algorithm has to be examined separately for each specific sparsification problem.

As an example, given a weighted undirected graph $G$ that is decomposed into edge-disjoint subgraphs, the goal of linear-sized subgraph sparsification is to construct a $(1+O(\varepsilon))$-spectral sparsifier $G^{\prime}$ to $G$, so that $G^{\prime}$ consists only of the reweighted versions of at most $n / \varepsilon^{2}$ given subgraphs. Our same algorithm for Theorem 1 runs in time $\widetilde{O}\left(m n^{1+1 / q} / \varepsilon^{5}\right)$ for this problem.

Weak Unweighted Graph Sparsification. Given $\kappa \in[1, m / n]$, consider the problem of finding a $\kappa$-spectral sparsifier of $G$ containing $O(m / \kappa)$ distinct edges from $E$, without reweighting. This problem is very recently studied by Anderson, Gu and Melgaard [AGM14], our regret minimization framework allows us to design a simple and almost-quadratic-time algorithm for this problem, improving from the quartic time complexity of [AGM14].

\section{Preliminaries}

Throughout this paper, for a cleaner representation that depends on the context, we interchangeably use $X \bullet Y=\langle X, Y\rangle=\operatorname{Tr}(X Y)$ to denote the inner product between two symmetric matrices. If $X$ is symmetric, we use $e^{X}$ to denote its matrix exponential and $\log X$ to denote its matrix logarithm, when $X$ is PSD. If $X$ is symmetric with eigendecomposition $X=\sum_{i=1}^{n} \lambda_{i} v_{i} v_{i}^{T}$ we denote by $|X| \stackrel{\text { def }}{=} \sum_{i=1}^{n}\left|\lambda_{i}\right| v_{i} v_{i}^{T}$. For any symmetric $X$, we use $\|X\|_{\text {spe }}$ to denote the spectral norm of $X$, and $\lambda_{\max }(X), \lambda_{\min }(X)$ to denote its largest and smallest eigenvalues. We define $\Delta_{n \times n} \stackrel{\text { def }}{=}\left\{X \in \mathbb{R}^{n \times n}\right.$ : $X \succeq 0, \operatorname{Tr} X=1\}$ to be the set of positive semidefinite (PSD) matrices with trace 1 . This should be seen as the matrix generalization of the $n$-dimensional simplex $\Delta_{n} \stackrel{\text { def }}{=}\left\{x \in \mathbb{R}^{n}: x \geq 0, \mathbb{1}^{T} x=1\right\}$. 
Regularizers and Bregman Divergence. We are interested in two types of regularizers over $\Delta_{n \times n}$, namely, $w(X) \stackrel{\text { def }}{=} X \bullet(\log X-I)$, known as the entropy regularizer, and $w(X) \stackrel{\text { def }}{=}$ $-\frac{q}{q-1} \operatorname{Tr} X^{1-1 / q}$ for some $q>1$, which we call the $\ell_{1-1 / q}$-regularizer. The corresponding Bregman divergences $V_{X}(Y) \stackrel{\text { def }}{=} w(Y)-w(X)-\langle\nabla w(X), Y-X\rangle$ are the following.

$$
\begin{aligned}
\text { entropy case: } & V_{X}(Y)=Y \bullet(\log Y-\log X)-I \bullet(Y-X), \\
\ell_{1-1 / q} \text { case: } & V_{X}(Y)=X^{-1 / q} \bullet Y+\frac{1}{q-1} \operatorname{Tr} X^{1-1 / q}-\frac{q}{q-1} \operatorname{Tr} Y^{1-1 / q} .
\end{aligned}
$$

Note that both regularizers above and their Bregman divergences are convex over the cone of PSD matrices. ${ }^{1}$ We now state some classical properties of Bregman divergence. Their proofs are included in Appendix D for completeness.

Lemma 2.1. The Bregman divergence of a convex differentiable function $w(\cdot)$ has the properties:

- Non-negativity: $V_{X}(Y) \geq 0$ for all $X, Y \geq 0$.

- The "three-point equality": $\langle\nabla w(X)-\nabla w(Y), X-U\rangle=V_{X}(U)-V_{Y}(U)+V_{Y}(X)$.

- Given $\tilde{X} \succeq 0$ and $X=\arg \min _{Z \in \Delta_{n \times n}} V_{\widetilde{X}}(Z)$ as the Bregman projection, we have the "generalized Pythagorean theorem" for all $U \in \Delta_{n \times n}: V_{\widetilde{X}}(U) \geq V_{X}(U)+V_{\widetilde{X}}(X) \geq V_{X}(U)$.

\section{Regret Minimization in Full Information}

In this section, we consider the following setting of the regret minimization problem, known as the full information setting. At each iteration $k=0, \ldots, T-1$, the player chooses an action $X_{k} \in \Delta_{n \times n}$, receives a symmetric loss matrix $F_{k} \in \mathbb{R}^{n \times n}$ and suffers a loss $\left\langle F_{k}, X_{k}\right\rangle$. At this point, the player is allowed to observe the full matrix $F_{k}$ without any restriction.

Again, the goal of the player is to minimize the regret with respect to any fixed matrix $U \in \Delta_{n \times n}$ :

$$
R(U) \stackrel{\text { def }}{=} \sum_{k=0}^{T-1}\left\langle F_{k}, X_{k}-U\right\rangle .
$$

The best choice of $U$ in hindsight can be taken as the rank-1 projection over a minimum eigenvector of $\sum_{k=0}^{T-1} F_{k}$. As a result, the total loss for the best choice of $U$ is $\lambda_{\min }\left(\sum_{k=0}^{T-1} F_{k}\right)$.

Entropy Regularizer. If $w(\cdot)$ is the entropy regularizer, then $(1.2)$ can be explicitly written as

$$
\text { MirrorDescent } \exp : \quad X_{k}=\exp ^{c I-\alpha \sum_{j=0}^{k-1} F_{j}} \text {, }
$$

where $c \in \mathbb{R}$ is the unique constant that ensures $\operatorname{Tr} X_{k}=1$. This is also known as the matrix multiplicative weight update method, and the following theorem gives its regret bound ${ }^{2}$

Theorem 3.1. In MirrorDescent $\mathrm{exp}_{\text {e }}$ if the parameter $\alpha>0$ satisfies $\alpha F_{k} \succeq-I$ for all iterations $k=0,1, \ldots, T-1$, then, for every $U \in \Delta_{n \times n}$,

$$
R(U) \stackrel{\text { def }}{=} \sum_{k=0}^{T-1}\left\langle F_{k}, X_{k}-U\right\rangle \leq \alpha \sum_{k=0}^{T-1}\left(X_{k} \bullet\left|F_{k}\right|\right) \cdot\left\|F_{k}\right\|_{\text {spe }}+\frac{V_{X_{0}}(U)}{\alpha} .
$$

We note that $V_{X_{0}}(U) \leq \log n$.

Our proof of Theorem 3.1 uses a technique known as the tweaked version of mirror descent (see [Zin03. Rak09]). We define an intermediate point $\widetilde{X}_{k+1}=\arg \min _{Z \succeq 0}\left\{V_{X_{k}}(Z)+\alpha\left\langle F_{k}, Z\right\rangle\right\}$ as the

\footnotetext{
${ }^{1}$ While this is easy to check by taking the second derivative for the entropy regularizer, it is less obvious for the $\ell_{1-1 / q}$ regularizer. The latter follows easily from Lieb's concavity theorem [Lie73, Bha97].

${ }^{2}$ The scalar version of this theorem was proved for instance in [AR09, Sha11, AO15]. A slightly different matrix version of this theorem was proved in [HKS12] (in particular, the authors of [HKS12] have required $I \succeq \alpha F_{k} \succeq-I$ while in fact it suffices to only require $\alpha F_{k} \succeq-I$.
} 
minimizer over $Z \succeq 0$, rather than $Z \in \Delta_{n \times n}$ as in (1.3). Accordingly, the actual point $X_{k+1}$ equals to $\arg \min _{Z \in \Delta_{n \times n}}\left\{V_{\widetilde{X}_{k+1}}(Z)\right\}$, the Bregman projection of $\widetilde{X}_{k+1}$ back to the hyperplane $\operatorname{Tr} Z=1$. This two-step interpretation of mirror descent gives a very clean proof to our regret bound, and we defer this full proof to Appendix E.

$\ell_{1-1 / q}$ regularizer. If $w(\cdot)$ is the $\ell_{1-1 / q}$ regularizer, then $(1.2)$ can be explicitly written as

$$
\text { MirrorDescent }_{\ell_{1-1 / \mathrm{q}}}: \quad X_{k}=\left(c I+\alpha \sum_{j=0}^{k-1} F_{j}\right)^{-q},
$$

where $c \in \mathbb{R}$ is the unique constant that ensures $c I+\alpha \sum_{j=0}^{k-1} F_{j} \succ 0$ and $\operatorname{Tr} X_{k}=1$.

If we focus on the special case of $q=2$ and each $F_{k}$ having rank 1, the following theorem gives the regret bound for MirrorDescent $\ell_{\ell_{1 / 2}}$.

Theorem 3.2. In MirrorDescent , $_{\ell_{1 / 2}}$ if the parameter $\alpha>0$, and the loss matrix $F_{k}$ is rank one and satisfies $X_{k}^{1 / 2} \bullet \alpha F_{k}>-1$ for all $k$, then, for every $U \in \Delta_{n \times n}$,

$$
R(U) \stackrel{\text { def }}{=} \sum_{k=0}^{T-1}\left\langle F_{k}, X_{k}-U\right\rangle \leq \alpha \cdot \sum_{k=0}^{T-1} \frac{\left(X_{k} \bullet F_{k}\right)\left(X_{k}^{1 / 2} \bullet F_{k}\right)}{1+X_{k}^{1 / 2} \bullet \alpha F_{k}}+\frac{V_{X_{0}}(U)}{\alpha} .
$$

If we instead have $X_{k}^{1 / 2} \bullet \alpha F_{k} \geq-\frac{1}{2}$, the above bound can be simplified as

$$
R(U) \stackrel{\text { def }}{=} \sum_{k=0}^{T-1}\left\langle F_{k}, X_{k}-U\right\rangle \leq 2 \alpha \cdot \sum_{k=0}^{T-1}\left(X_{k} \bullet F_{k}\right)\left(X_{k}^{1 / 2} \bullet F_{k}\right)+\frac{V_{X_{0}}(U)}{\alpha} .
$$

We note that $V_{X_{0}}(U) \leq 2 \sqrt{n}$.

We recommend the interested readers to see the proof of Theorem 3.2 in Appendix E, as it provides a straightforward generalization of Theorem 3.1 using regularizers other than entropy.

Theorem 3.2 is only a special case of the following more general regret bound, which holds for arbitrary $q \geq 2$, and for $F_{k}$ having arbitrary rank. At a first reading, one can skip Theorem 3.3 because its sole purpose in this paper is to improve the running time of graph sparsification from $\widetilde{O}\left(m n^{3 / 2}\right)$ to $\widetilde{O}\left(m n^{1+1 / q}\right)$, as well as allowing one to sparsify sums of high rank PSDs.

Theorem 3.3. In MirrorDescent $\ell_{\ell_{1-1}}$ with $q \geq 2$ and $\alpha>0$, if the loss matrix $F_{k}$ is either positive or negative semidefinite and satisfies $\alpha X_{k}^{1 / 2 q} F_{k} X_{k}^{1 / 2 q} \succeq-\frac{1}{2 q} I$ for all $k$, then for every $U \in \Delta_{n \times n}$,

$$
R(U) \stackrel{\text { def }}{=} \sum_{k=0}^{T-1}\left\langle F_{k}, X_{k}-U\right\rangle \leq O(q \alpha) \sum_{k=0}^{T-1}\left(X_{k} \bullet\left|F_{k}\right|\right) \cdot\left\|X_{k}^{1 / 2 q} F_{k} X_{k}^{1 / 2 q}\right\|_{\text {spe }}+\frac{V_{X_{0}}(U)}{\alpha} .
$$

We note that $V_{X_{0}}(U) \leq \frac{q}{q-1} n^{1 / q}$.

(The proof of Theorem 3.3 is deferred to Appendix E.)

The key idea to prove Theorem 3.3 is to replace the use of the Sherman-Morrison formula in the proof of Theorem 3.2 with the Woodbury formula so as to allow $F_{k}$ to be of high rank. It also uses the Lieb-Thirring trace inequality to handle arbitrary $q \geq 2$.)

\section{Warm-Up: Upper-Sided Linear-Sized Sparsification}

In this section and the next, we present our construction of linear-sized sparisifier in the general matrix setting. Its specialization to graph sparsification appears in Appendix B while its efficient implementation is discussed in Section 6. To showcase how the regret bounds of Section 3 can be useful in the construction of sparsifiers, we start by describing a warm-up example in which we are 
only interested in obtaining a single side of the sparsification guarantee.

Suppose we are given a decomposition of the identity matrix $I=\sum_{e=1}^{m} w_{e} \widehat{L}_{e}$, where each $\widehat{L}_{e}$ satisfies

$0 \preceq \widehat{L}_{e} \preceq I$ and is of rank 1 and trace 1 , i.e. $\widehat{L}_{e}=v v^{t}$ for some $v \in \mathbb{R}^{n}$ with $\|v\|_{2}=1$.

The weights $w_{e}>0$ may be unknown, though the trace guarantee ensures that $\sum_{e} w_{e}=n$. In this section, we are interested in finding some $s \in \Delta_{m}$ satisfying $\sum_{e=1}^{m}\left(n s_{e}\right) \cdot \widehat{L}_{e} \preceq(1+\varepsilon) I$, while the sparsity of $s$ - that is, $\left|\left\{e \in[m]: s_{e}>0\right\}\right|$ - is at most $O\left(n / \varepsilon^{2}\right)$. We call this the uppersided linear-sized spectral sparsification because it only gives an upper bound on the eigenvalues of $\sum_{e=1}^{m}\left(n s_{e}\right) \cdot \widehat{L}_{e}$ and no lower bound.

Consider the following algorithm that invokes the regret minimization framework in Section 3 to solve this upper-sided sparsification. We choose

$$
\text { the } \ell_{1 / 2} \text { regularizer and } \alpha=\varepsilon / 4 \sqrt{n} \text { for MirrorDescent } \ell_{\ell_{1 / 2}} \text {. }
$$

At iteration $k$, set the feedback matrix as $F_{k}=-n \widehat{L}_{e_{k}}$, where $e_{k}$ minimizes $\widehat{L}_{e} \bullet X_{k}$ over $e \in[m]{ }^{3}$

Before applying Theorem 3.2. let us first verify that the prerequisite $X_{k}^{1 / 2} \bullet \alpha F_{k} \geq-\frac{1}{2}$ holds. Because $\sum_{e \in[m]} \frac{w_{e}}{n} \widehat{L}_{e} \bullet X_{k}=\frac{1}{n} I \bullet X_{k}=\frac{1}{n}$, by an averaging argument, we must have $\widehat{L}_{e_{k}} \bullet X_{k} \leq \frac{1}{n}$. This further implies $-\alpha n \widehat{L}_{e_{k}} \bullet X_{k}^{1 / 2} \geq-\alpha \sqrt{n}>-\frac{1}{2}$ due to the claim below.

Claim 4.1. For every $X \in \Delta_{n \times n}$, we have $\widehat{L}_{e} \bullet X^{1 / 2} \leq\left(\widehat{L}_{e} \bullet X\right)^{1 / 2}$ for every $e \in[m]$.

Proof. Without loss of generality, one can assume $X$ to be diagonal. Next, since $\widehat{L}_{e}=v_{e} v_{e}^{T}$ is of rank one, the desired inequality follows from Jensen's inequality $v_{e}^{T} X^{1 / 2} v_{e} \leq \sqrt{v_{e}^{T} X v_{e}}$ and the fact that $\left\|v_{e}\right\|_{2}^{2}=\operatorname{Tr} \widehat{L}_{e} \leq 1$.

Now, applying Theorem 3.2. we obtain that for every $U \in \Delta_{n \times n}$,

$$
\sum_{k=0}^{T-1}\left\langle-n \widehat{L}_{e_{k}}, X_{k}-U\right\rangle \leq 2 \alpha \cdot \sum_{k=0}^{T-1}\left(X_{k} \bullet n \widehat{L}_{e_{k}}\right)\left(X_{k}^{1 / 2} \bullet n \widehat{L}_{e_{k}}\right)+\frac{2 \sqrt{n}}{\alpha} .
$$

After rearranging, and using $\widehat{L}_{e_{k}} \bullet X_{k} \leq \frac{1}{n}$ and $n \widehat{L}_{e_{k}} \bullet X_{k}^{1 / 2} \leq \sqrt{n}$ we deduced earlier,

$$
\begin{aligned}
\left\langle\frac{n}{T} \sum_{k=0}^{T-1} \widehat{L}_{e_{k}}, U\right\rangle & \leq \frac{2 \alpha}{T} \cdot \sum_{k=0}^{T-1}\left(X_{k} \bullet n \widehat{L}_{e_{k}}\right)\left(X_{k}^{1 / 2} \bullet n \widehat{L}_{e_{k}}\right)+\frac{1}{T} \sum_{k}\left\langle n \widehat{L}_{e_{k}}, X_{k}\right\rangle+\frac{2 \sqrt{n}}{\alpha T} \\
& \leq \frac{2 \alpha}{T} \cdot T \cdot 1 \cdot \sqrt{n}+1+\frac{2 \sqrt{n}}{\alpha T}=\frac{\varepsilon}{2}+1+\frac{8 n}{\varepsilon T} .
\end{aligned}
$$

Finally, choosing $T=16 n / \varepsilon^{2}$ and $U$ to be the rank-1 projection over a maximum eigenvector, we conclude that $\lambda_{\max }\left(\frac{n}{T} \sum_{k=0}^{T-1} \widehat{L}_{e_{k}}\right) \leq 1+\varepsilon$.

This completes the description of our upper-sided linear-sized sparsification algorithm. The full sparsification algorithm, in the next section, will essentially consists of playing out this analysis on the lower and upper side at the same time.

We emphasize here that if one chooses the entropy regularizer by using MirrorDescent $t_{\text {exp }}$ and chooses $e_{k}=e$ with probability proportional to $w_{e}$, a similar analysis from the one above recovers the sparsification result of Spielman and Srivastava [SS11].

\footnotetext{
${ }^{3}$ This choice naturally follows from a saddle-point interpretation of the problem, because it is the subgradient of the function $f(X) \stackrel{\text { def }}{=} \min _{s \in \Delta_{m}} \sum_{e=1}^{m}\left(n s_{e} \widehat{L}_{e}\right) \bullet X$ at $X=X_{k}$. We have skipped the explanation of this choice due to the space limitation.
} 


\section{Linear-Sized Sparsification}

As before, suppose we are given a decomposition of the identity matrix $I=\sum_{e=1}^{m} w_{e} \widehat{L}_{e}$, where each $\widehat{L}_{e}$ satisfies $0 \preceq \widehat{L}_{e} \preceq I$ and is of rank 1 and trace 1 . The weights $w_{e}>0$ may be unknown and satsify $\sum_{e} w_{e}=n$. In this section, we are interested in finding scalars $s_{e} \geq 0$ satisfying

$$
I \preceq \sum_{e=1}^{m} s_{e} \cdot \widehat{L}_{e} \preceq\left(1+8 \varepsilon+O\left(\varepsilon^{2}\right)\right) I,
$$

while the sparsity of $s$ - that is, $\left|\left\{e \in[m]: s_{e}>0\right\}\right|$ - is at most $O\left(n / \varepsilon^{2}\right)$.

Instead of maintaining one sequence $X_{k}$ like in Section 4. we maintain two sequences $X_{k}, Y_{k} \in$ $\Delta_{n \times n}$. At each iteration $k \in 0,1, \ldots, T-1$, find an arbitrary $e_{k} \in[m]$ such that

$$
\widehat{L}_{e_{k}} \bullet X_{k} \leq \widehat{L}_{e_{k}} \bullet Y_{k} \text {. }
$$

This is always possible by an averaging argument with weights $w_{e}$. Next, we choose the $\ell_{1 / 2}$ regularizer and some parameter $\alpha<1 / 2$ (in fact, we will choose $\alpha=\varepsilon$ later), and updates

$$
\begin{aligned}
& X_{k+1}=\underset{Z \in \Delta_{n \times n}}{\arg \min }\left\{V_{X_{k}}(Z)+\left\langle\frac{-\alpha \widehat{L}_{e_{k}}}{\left(X_{k} \bullet \widehat{L}_{e_{k}}\right)^{1 / 2}}, Z\right\rangle\right\} \text { and } \\
& Y_{k+1}=\underset{Z \in \Delta_{n \times n}}{\arg \min }\left\{V_{Y_{k}}(Z)+\left\langle\frac{\alpha \widehat{L}_{e_{k}}}{\left(Y_{k} \bullet \widehat{L}_{e_{k}}\right)^{1 / 2}}, Z\right\rangle\right\} .
\end{aligned}
$$

In other words, we have picked feedback matrices $F_{k}=\frac{-\widehat{L}_{e_{k}}}{\left(X_{k} \bullet \widehat{L}_{e_{k}}\right)^{1 / 2}}$ for the $X_{k}$ sequence and $F_{k}=$ $\frac{\widehat{L}_{e_{k}}}{\left(Y_{k} \bullet \widehat{L}_{e_{k}}\right)^{1 / 2}}$ for the $Y_{k}$ sequence in our MirrorDescent $\ell_{\ell_{1 / 2}} 4$

Notice that $X_{k}^{1 / 2} \bullet \frac{-\alpha \widehat{L}_{e_{k}}}{\left(X_{k} \bullet \widehat{L}_{e_{k}}\right)^{1 / 2}} \geq-\frac{1}{2}$ due to Claim 4.1. so we always have $X_{k}^{1 / 2} \bullet \alpha F_{k} \geq-\frac{1}{2}$ which satisfies the prerequisite of Theorem 3.2. Applying Theorem 3.2 on the $X_{k}$ sequence, we obtain that for every $U_{X} \in \Delta_{n \times n}$,

$$
\begin{aligned}
\sum_{k=0}^{T-1}\left\langle\frac{-\widehat{L}_{e_{k}}}{\left(X_{k} \bullet \widehat{L}_{e_{k}}\right)^{1 / 2}}, X_{k}-U_{X}\right\rangle & \leq 2 \alpha \cdot \sum_{k=0}^{T-1}\left(X_{k} \bullet \frac{\widehat{L}_{e_{k}}}{\left(X_{k} \bullet \widehat{L}_{e_{k}}\right)^{1 / 2}}\right)\left(X_{k}^{1 / 2} \bullet \frac{\widehat{L}_{e_{k}}}{\left(X_{k} \bullet \widehat{L}_{e_{k}}\right)^{1 / 2}}\right)+\frac{V_{X_{0}}\left(U_{X}\right)}{\alpha} \\
& =2 \alpha \cdot \sum_{k=0}^{T-1} X_{k}^{1 / 2} \bullet \widehat{L}_{e_{k}}+\frac{V_{X_{0}}\left(U_{X}\right)}{\alpha} \leq 2 \alpha \cdot \sum_{k=0}^{T-1}\left(X_{k} \bullet \widehat{L}_{e_{k}}\right)^{1 / 2}+\frac{V_{X_{0}}\left(U_{X}\right)}{\alpha} .
\end{aligned}
$$

Above, the last inequality uses Claim 4.1. If we denote by $M_{X} \stackrel{\text { def }}{=} \sum_{k=0}^{T-1} \frac{\widehat{L}_{e_{k}}}{\left(\widehat{L}_{e_{k}} \bullet X_{k}\right)^{1 / 2}}$ and rearrange the inequality above, we get

$$
M_{X} \bullet U_{X} \leq \frac{V_{X_{0}}\left(U_{X}\right)}{\alpha}+(1+2 \alpha) \sum_{k=0}^{T-1}\left(\widehat{L}_{e_{k}} \bullet X_{k}\right)^{1 / 2} .
$$

Similarly, applying Theorem 3.2 on the $Y_{k}$ sequence, and define $M_{Y} \stackrel{\text { def }}{=} \sum_{k=0}^{T-1} \frac{\widehat{L}_{e_{k}}}{\left(\widehat{L}_{e_{k}} \bullet Y_{k}\right)^{1 / 2}}$, we obtain that for every $U_{Y} \in \Delta_{n \times n}$,

$$
M_{Y} \bullet U_{Y} \geq-\frac{V_{Y_{0}}\left(U_{Y}\right)}{\alpha}+(1-2 \alpha) \sum_{k=0}^{T-1}\left(\widehat{L}_{e_{k}} \bullet Y_{k}\right)^{1 / 2} .
$$

In the rest of the proof, we will use (5.3) and (5.4) to deduce

$$
\lambda_{\max }\left(M_{Y}\right)-\lambda_{\min }\left(M_{Y}\right) \leq 8 \varepsilon(1+O(\varepsilon)) \lambda_{\min }\left(M_{Y}\right) .
$$

\footnotetext{
${ }^{4}$ In fact, the denominator $\left(X_{k} \bullet \widehat{L}_{e_{k}}\right)^{1 / 2}$ is defined so as to make sure that $F_{k}$ is the 'maximally aggressive' loss matrix we can have for MirrorDescent $\ell_{\ell_{1 / 2}}$.
} 
Finally, since $M_{Y}=\sum_{k=0}^{T-1} \frac{\widehat{L}_{e_{k}}}{\left(\widehat{L}_{e_{k}} \bullet \cup_{k}\right)^{1 / 2}}$ is a matrix that is a summation of at most $T=n / \varepsilon^{2}$ rank-1 matrices, dividing it by $\lambda_{\min }\left(M_{Y}\right)$ gives the desired sparsification for (5.1).

We prove (5.5) in two steps.

Lowerbounding $\boldsymbol{\lambda}_{\min }\left(\boldsymbol{M}_{\boldsymbol{Y}}\right)$. Recall that we have $\operatorname{Tr}\left(M_{X}\right)=\sum_{k=0}^{T-1} \frac{1}{\left(\widehat{L}_{e} \bullet X_{k}\right)^{1 / 2}}$ because we have assumed each $\widehat{L}_{e}$ to be of trace 1 . Denoting by $a_{k}=\left(\widehat{L}_{e} \bullet X_{k}\right)^{1 / 2}$, we have that $\operatorname{Tr}\left(M_{X}\right)=\sum_{k=0}^{T-1} \frac{1}{a_{k}}$. We apply (5.3) here with $U_{X}=\frac{1}{n} I=X_{0}$, and obtain

$$
\frac{1}{n} \sum_{k=0}^{T-1} \frac{1}{a_{k}}=\frac{1}{n} \operatorname{Tr}\left(M_{X}\right) \leq(1+2 \alpha) \sum_{k=0}^{T-1}\left(\widehat{L}_{e} \bullet X_{k}\right)^{1 / 2} \leq(1+2 \alpha) \sum_{k=0}^{T-1} a_{k} .
$$

Applying Cauchy-Schwarz, we have

$$
\left(\sum_{k=0}^{T-1} a_{k}\right)^{2} \geq \frac{1}{n(1+2 \alpha)}\left(\sum_{k=0}^{T-1} a_{k}\right)\left(\sum_{k=0}^{T-1} \frac{1}{a_{k}}\right) \geq \frac{T^{2}}{n(1+2 \alpha)} .
$$

If we choose $T=\frac{n}{\varepsilon^{2}}$, we immediately have ${ }^{5}$

$$
\sum_{k=0}^{T-1}\left(\widehat{L}_{e} \bullet Y_{k}\right)^{1 / 2} \geq \sum_{k=0}^{T-1} a_{k} \geq \frac{\sqrt{n}}{\varepsilon^{2}}(1-O(\alpha)) .
$$

Substituting the above lower bound into (5.4), and choosing $U_{Y} \in \Delta_{n \times n}$ to be the rank-1 projection matrix over the smallest eigenvector of $M_{Y}$, and choosing $\alpha=\varepsilon$, we have

$$
\lambda_{\min }\left(M_{Y}\right) \geq-\frac{2 \sqrt{n}}{\alpha}+(1-2 \alpha) \sum_{k=0}^{T-1}\left(\widehat{L}_{e} \bullet Y_{k}\right)^{1 / 2} \geq(1-O(\varepsilon)) \frac{\sqrt{n}}{\varepsilon^{2}}
$$

Upperbounding $\lambda_{\max }\left(M_{\boldsymbol{Y}}\right)-\boldsymbol{\lambda}_{\min }\left(\boldsymbol{M}_{\boldsymbol{Y}}\right)$. This time, we use our choice of $\widehat{L}_{e_{k}} \bullet X_{k} \leq \widehat{L}_{e_{k}} \bullet Y_{k}$ to combine (5.3) and (5.4) and derive that

$$
\frac{1}{1+2 \alpha} M_{Y} \bullet U_{X} \leq \frac{1}{1+2 \alpha} M_{X} \bullet U_{X} \leq \frac{1}{1-2 \alpha} M_{Y} \bullet U_{Y}+\frac{2 \sqrt{n}}{\alpha}\left(\frac{1}{1+2 \alpha}+\frac{1}{1-2 \alpha}\right) .
$$

Choosing $U_{X}$ to be the rank-1 matrix projection matrix over the largest eigenvector of $M_{Y}, U_{Y}$ to be that over the smallest eigenvector of $M_{Y}$, and recalling that $\alpha=\varepsilon$, we have

$$
\lambda_{\max }\left(M_{Y}\right) \leq \frac{1+2 \varepsilon}{1-2 \varepsilon} \lambda_{\min }\left(M_{Y}\right)+\frac{4 \sqrt{n}}{\varepsilon}(1+O(\varepsilon)) .
$$

After rearranging and substituting in the lower bound (5.7), we finish the proof of (5.5)

$$
\lambda_{\max }\left(M_{Y}\right)-\lambda_{\min }\left(M_{Y}\right) \leq \frac{4 \varepsilon}{1-2 \varepsilon} \lambda_{\min }\left(M_{Y}\right)+\frac{4 \sqrt{n}}{\varepsilon}(1+O(\varepsilon)) \leq 8 \varepsilon(1+O(\varepsilon)) \lambda_{\min }\left(M_{Y}\right) .
$$

\section{Efficient Implementation for Graph Sparsification}

The update rules described in (5.2) imply that $X_{k}$ and $Y_{k}$ are of the form (see Section 3)

$$
X_{k}=\left(c^{X} \cdot I-\sum_{j=0}^{k-1} s_{j}^{X} \widehat{L}_{e_{j}}\right)^{-2} \text { and } Y_{k}=\left(\sum_{j=0}^{k-1} s_{j}^{Y} \widehat{L}_{e_{j}}-c^{Y} \cdot I\right)^{-2} .
$$

Here, $c^{X}$ is the unique (positive) constant that satisfies $c^{X} I-\sum_{j=0}^{k-1} s_{j}^{X} \widehat{L}_{e_{j}} \succ 0$ and $\operatorname{Tr} X_{k}=1$, while $c^{Y}$ is the unique (possibly negative) constant that satisfies $\sum_{j=0}^{k-1} s_{j}^{Y} \widehat{L}_{e_{j}}-c^{Y} I \succ 0$ and $\operatorname{Tr} Y_{k}=1$.

\footnotetext{
${ }^{5}$ In fact, it suffices to stop our algorithm at the earliest iteration $T$ so that inequality $(5.6)$ is satisfied. Our analysis here only represents the most pessimistic scenario; in practice, this early termination implies we can choose less than $n / \varepsilon^{2}$ matrices for certain inputs. This is in contrast to [BSS14], as their algorithm uses $n / \varepsilon^{2}$ rank-1 matrices for all inputs.
} 
The coefficients $s_{j}^{X}$ and $s_{j}^{Y}$ are always positive. (It is worth noting that $c^{X}$ is initially $\sqrt{n}$ at $X_{0}$ and keeps increasing, while $c^{Y}$ is initially $-\sqrt{n}$ and keeps increasing as well.)

Recall that MirrorDescent $\ell_{\ell_{1 / 2}}$ requires one to compute $c^{X}$ and $c^{Y}$ for each iteartion, and this can be done via binary search. One way to perform binary search is to first compute $\lambda_{\max }=$ $\lambda_{\max }\left(\sum_{j=0}^{k-1} s_{j}^{X} \widehat{L}_{e_{j}}\right)$. Then, one can binary search $c^{X}$ in the range $\left[\lambda_{\max }+1, \lambda_{\max }+\sqrt{n}\right]$ to find the correct one satisfying $\operatorname{Tr}\left(c^{X} \cdot I-\sum_{j=0}^{k-1} s_{j}^{X} \widehat{L}_{e_{j}}\right)^{-2}=1$. Similarly, one can binary search $c^{Y}$ in the range of $\left[\lambda_{\min }-\sqrt{n}, \lambda_{\min }-1\right]$ where $\lambda_{\min }=\lambda_{\min }\left(\sum_{j=0}^{k-1} s_{j}^{Y} \widehat{L}_{e_{j}}\right)^{6}$

If one performs the binary search to an accuracy that is small enough, this gives an algorithm whose running time is $\widetilde{O}\left(n^{3} m / \varepsilon^{2}\right)$, dominated by the computation of $X_{k} \bullet \widehat{L}_{e}=\left(c^{X} \cdot I-\right.$ $\left.\sum_{j=0}^{k-1} s_{j}^{X} \widehat{L}_{e_{j}}\right)^{-2} \bullet \widehat{L}_{e}$ for each $k \in[T]$ and $e \in[m]$.

Running Time Improvement. For the graph sparsification problem described in Theorem 1. we sketch the key ideas needed to improve the running time to $\widetilde{O}\left(m n^{1+1 / q} / \varepsilon^{5}\right)$ for any even integer $q \geq 2$. The details can be found in Appendix F and G. In particular, we first describe how to achieve a running time of $\widetilde{O}\left(m n^{1+1 / 2} / \varepsilon^{5}\right)$.

Recall that in Section 5. we have constructed $M_{X}$ and $M_{Y}$ and proved that $\lambda_{\min }\left(M_{X}\right)$ and $\lambda_{\min }\left(M_{Y}\right)$ are both at least $\Omega\left(\sqrt{n} / \varepsilon^{2}\right)$. In fact, it is not hard to ensure that $\lambda_{\max }\left(M_{X}\right)$ and $\lambda_{\max }\left(M_{Y}\right)$ are at most $O\left(\sqrt{n} / \varepsilon^{2}\right)$ as well. 7 Since $\sum_{j=0}^{k-1} s_{j}^{X} \widehat{L}_{e_{j}} \preceq \alpha M_{X}$, we conclude that the eigenvalues of $\sum_{j=0}^{k-1} s_{j}^{X} \widehat{L}_{e_{j}}$ are all upper bounded by $\alpha \cdot O\left(\sqrt{n} / \varepsilon^{2}\right)=O(\sqrt{n} / \varepsilon)$. Therefore, throughout the algorithm, the encountered choices of $c^{X}$ are always upper bounded by $O(\sqrt{n} / \varepsilon)$.

For this reason, we only need to compute matrix inversions of the form $(c I-A)^{-1}$, with the guarantee that $c=O(\sqrt{n} / \varepsilon)$. Since we always have $c I-A \succeq I$-as otherwise $\operatorname{Tr}(c I-A)^{-2}$ is strictly larger than 1 - we can approximate this matrix inverse by

$$
(c I-A)^{-1}=c^{-1}\left(I-\frac{A}{c}\right)^{-1} \approx c^{-1}\left(I+\frac{A}{c}+\frac{A^{2}}{c^{2}}+\cdots \frac{A^{d}}{c^{d}}\right),
$$

and it suffices to choose the maximum degree $d=O(\sqrt{n} / \varepsilon)$. This is formally proved in Lemma G.6. In other words, when computing $X_{k}$, it suffices to replace the matrix inversion with some matrix polynomial of degree $d=O(\sqrt{n} / \varepsilon)$. Similar idea also holds for the $Y_{k}$ sequence.

So far, we managed avoiding the computationally expensive matrix inversion. Next, we want to further accelerate the procedure of computing $(c I-A)^{-2} \bullet \widehat{L}_{e}$ for all edges $e \in[m]$ simultaneously. Recall that $\widehat{L}_{e}=v_{e} v_{e}^{T}$ is of rank 1 , and one can rewrite

$$
(c I-A)^{-2} \bullet \widehat{L}_{e}=v_{e}^{T}(c I-A)^{-2} v_{e}=\left\|(c I-A)^{-1} v_{e}\right\|_{2}^{2} .
$$

For this reason, as in [SS11], one can apply the Johnson-Lindenstrauss dimension reduction [JL84]: there exists random matrix $Q$ with $\widetilde{O}\left(1 / \varepsilon^{2}\right)$ rows, satisfying that $\left\|(c I-A)^{-1} v_{e}\right\|_{2}^{2} \approx \| Q(c I-$ $A)^{-1} v_{e} \|_{2}^{2}$ for for all $v_{e}$.

Using this dimension reduction, one can precompute $T=Q(c I-A)^{-1}$ in time $\widetilde{O}\left(m / \varepsilon^{2}\right) \times$ $\widetilde{O}(\sqrt{n} / \varepsilon)=\widetilde{O}\left(m \sqrt{n} / \varepsilon^{3}\right)$, with the help from the approximate matrix inversion (6.2), and the nearlylinear time Laplacian system solvers [ST04]. After the precomputation, each $(c I-A)^{-2} \bullet \widehat{L}_{e} \approx$ $\left\|T v_{e}\right\|_{2}^{2}$ can be computed in $\widetilde{O}\left(1 / \varepsilon^{2}\right)$ time, totaling $\widetilde{O}\left(m / \varepsilon^{2}\right)$ per iteration, which is negligible.

In sum, taking into account that we have $T=n / \varepsilon^{2}$ iterations, the total running time is $\widetilde{O}\left(m n^{1+1 / 2} / \varepsilon^{5}\right)$. To turn this $\widetilde{O}\left(m n^{1+1 / 2} / \varepsilon^{5}\right)$ into $\widetilde{O}\left(m n^{1+1 / q} / \varepsilon^{5}\right)$ for any constant $q$, we need to replace the use of the $\ell_{1 / 2}$ regularizer with the $\ell_{1-1 / q}$ regularizer. This requires one to use Theorem 3.3 in replacement of Theorem 3.2.

\footnotetext{
${ }^{6} \lambda_{\max }$ and $\lambda_{\min }$ can be computed via power methods, and it suffices to compute them up to an additive error of, say, 0.1. In Appendix G, we propose an alternative approach to compute $c^{X}$ and $c^{Y}$, avoiding the use of power methods.

${ }^{7}$ This may require one to stop the algorithm earlier than $T=n / \varepsilon^{2}$ iterations, which is even better!
} 
We wish to emphasize here that our analysis in Section 5 needs to be strengthened in order to tolerate all the errors incurred from the approximate computations (most notably from Laplacian linear solvers, from Johnson-Lindenstrauss, and from (6.2)). This is only rountinary thanks to the optimization motivation behind our argument, and we have done this carefully in Appendix F.

\section{Acknowledgement}

We thank Richard Peng, Nikhil Srivastava, and Nisheeth Vishnoi for helpful conversations. This material is based upon work partly supported by the National Science Foundation under Grant CCF-1319460 and by a Simons Graduate Student Award under grant no. 284059. 


\section{APPENDIX}

\section{Appendix roadmap.}

- In Figure 1, we plot the entropy and the $\ell_{1 / 2}$ regularizers of the 3 -dimensional scalar case for a visual comparison.

- In Appendix A, we verify the equivalence between FTRL and MirrorDescent for our choices of the regularizers.

- In Appendix B, we provide notations for graphs, and state the reduction from the sparsifying graphs to sparsifying sums of rank-1 matrices.

- In Appendix C, we provide our unweighted sparsification result.

- In Appendix D and E we provide missing proofs for Section 2 and 3 respectively.

- In Appendix F, we generalize our sparsification algorithm of Section 5 to allow arbitrary $q \geq 2$, high rank matrices, and approximate computations.

- In Appendix G. we provide the details of how to implement linear-sized graph sparsifications in almost-quadratic time, thus finishing the running time claim of Theorem 1.

- In Appendix H, we sketch how to generalize our running time improvement to other problems, including sparsifying sums of rank-1 PSD matrices (i.e., Theorem F.5), as well as subgraph sparsifications.

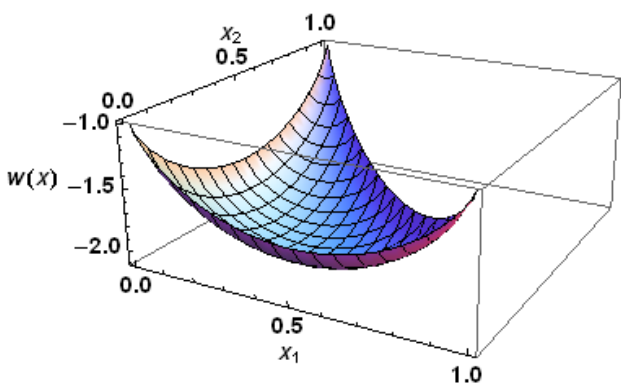

(a) The entropy regularizer

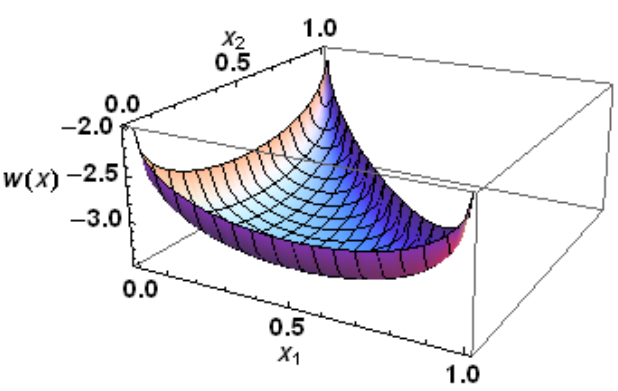

(b) The $\ell_{1 / 2}$ regularizer

Figure 1: Two regularizers in $n=3$. The first two axes represent $x_{1}, x_{2}$ so $x_{3}=1-x_{1}-x_{2}$. The third axes represent $w(x)$. 


\section{A Partial Equivalence Between FTRL and Mirror Descent}

In this section, we show the equivalence between mirror descent and follow-the-regularized-leader for our choices of the regularizers. In fact, this equivalence holds more generally for all regularizers $w(\cdot)$ that are convex function of Legendre type with domain $Q$ (see for instance [BMDG05. Roc96]).

Letting $A_{i} \in \mathbb{R}^{n}$ be any symmetric matrix for each iteration $i$, the follow-the-regularized-leader method can be described as

$$
\forall k=0,1, \ldots, T-1, \quad X_{k}=\underset{Z \in \Delta_{n \times n}}{\arg \min }\left\{w(Z)+\sum_{i=0}^{k-1}\left\langle A_{i}, Z\right\rangle\right\} .
$$

The mirror descent method (with starting point $\widetilde{X}_{0}=\frac{1}{n} I$ ) can be described as

$$
\forall k=0,1, \ldots, T-1, \quad \widetilde{X}_{k}=\underset{Z \in \Delta_{n \times n}}{\arg \min }\left\{V_{\widetilde{X}_{k-1}}(Z)+\left\langle A_{k-1}, Z\right\rangle\right\},
$$

where as before, $V_{X}(Y) \stackrel{\text { def }}{=} w(Y)-\langle\nabla w(X), Y-X\rangle-w(X)$ is the Bregman divergence of $w(\cdot)$.

Recall that when $w(X)=X \bullet(\log X-I)$ is the entropy regularizer, then $\nabla w(X)=\log X$ and therefore $(\nabla w)^{-1}(A)=e^{A}$. When $w(X)=-\frac{q}{q-1} \operatorname{Tr} X^{1-1 / q}$ is the $\ell_{1-1 / q}$ regularizer, then $\nabla w(X)=X^{-1 / q}$ and therefore $(\nabla w)^{-1}(A)=A^{-q}$. The rest of the proof holds for both these two types of regularizers.

To compute the minimizer $X_{k}$ for (A.1), one can take the derivative and demand that $\nabla w\left(X_{k}\right)+$ $\sum_{i=0}^{k-1} A_{i}-c_{k} \cdot I=0$. Here, the extra term $-c_{k} \cdot I$ comes from the Lagrange multipliers of the linear constraint $\operatorname{Tr}(Z)=I \bullet Z=1$. (We do not have Lagrange multipliers for the other constraint $Z \succeq 0$ because our gradient $\nabla w(Z)$ is a barrier function and tends to infinite as any eigenvalue of $Z$ tends to zero.) It is now easy to see that $c_{k}$ is the unique constant that ensures $\sum_{i=0}^{k-1} A_{i}-c_{k} I \preceq 0$ (because $\left.\nabla w\left(X_{k}\right) \succeq 0\right)$ and that $\operatorname{Tr} X_{k}=\operatorname{Tr}\left((\nabla w)^{-1}\left(c_{k} I-\sum_{i=0}^{k-1} A_{i}\right)\right)=1$.

To compute the minimizer $X_{k}$ for (A.2), one can take the derivative and demand that $\nabla w\left(\widetilde{X}_{k}\right)-$ $\nabla w\left(\widetilde{X}_{k-1}\right)+A_{i}-d_{k} \cdot I=\nabla V_{\widetilde{X}_{k-1}}\left(\widetilde{X}_{k}\right)+A_{i}-d_{k} \cdot I=0$. Here, the extra term $-d_{k} \cdot I$ again comes from the Lagrange multipliers of the linear constraint $\operatorname{Tr}(Z)=I \bullet Z=1$. It is now easy to see that $d_{k}$ is the unique constant that ensures $-\nabla w\left(\widetilde{X}_{k-1}\right)+A_{i}-d_{k} \cdot I \preceq 0$ (because $\left.\nabla w\left(\widetilde{X}_{k}\right) \succeq 0\right)$ and that $\operatorname{Tr} \widetilde{X}_{k}=\operatorname{Tr}\left((\nabla w)^{-1}\left(\nabla w\left(\widetilde{X}_{k-1}\right)+d_{k} I-A_{k-1}\right)\right)=1$.

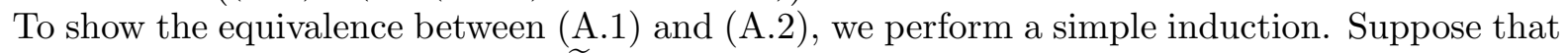
$\widetilde{X}_{k-1}=X_{k-1}$, and we wish to prove $\widetilde{X}_{k}=X_{k}$.

In this case, we have

$$
\begin{aligned}
\widetilde{X}_{k} & =(\nabla w)^{-1}\left(\nabla w\left(\widetilde{X}_{k-1}\right)+d_{k} I-A_{k-1}\right)=(\nabla w)^{-1}\left(\nabla w\left(X_{k-1}\right)+d_{k} I-A_{k-1}\right) \\
& =(\nabla w)^{-1}\left(c_{k-1} I+d_{k} I-\sum_{i=0}^{k-1} A_{i}\right), \text { and } \\
X_{k} & =(\nabla w)^{-1}\left(c_{k} I-\sum_{i=0}^{k-1} A_{i}\right) .
\end{aligned}
$$

Finally, since $d_{k}$ is the unique constant that ensures $c_{k-1} I+d_{k} I-\sum_{i=0}^{k-1} A_{i} \succeq 0$ and $\operatorname{Tr}\left((\nabla w)^{-1}\left(c_{k-1} I+\right.\right.$ $\left.\left.d_{k} I-\sum_{i=0}^{k-1} A_{i}\right)\right)=1$, while $c_{k}$ is the unique constant that ensures $c_{k} I-\sum_{i=0}^{k-1} A_{i} \succeq 0$ and $\operatorname{Tr}\left((\nabla w)^{-1}\left(c_{k} I-\sum_{i=0}^{k-1} A_{i}\right)\right)=1$, it is obvious to see that $c_{k}=c_{k-1}+d_{k}$ and therefore $\widetilde{X}_{k}=X_{k}$.

\section{B Graph Notations}

Let $G=(V, E, w)$ be a undirected weighted graph with $n$ vertices and $m$ edges, and each $w_{e}>0$ is the weight of edge $e$. Without loss of generality, we study only connected graphs throughout this 
paper. For every edge $e=(a, b) \in E$, we orient it arbitrarily and denote by $\chi_{e} \stackrel{\text { def }}{=} \mathbf{e}_{a}-\mathbf{e}_{b} \in \mathbb{R}^{n}$ the characteristic (column) vector of edge $e$.

Let $L_{e} \stackrel{\text { def }}{=} w_{e} \chi_{e} \chi_{e}^{T} \in \mathbb{R}^{n \times n}$ be the graph Laplacian of edge $e$, or the edge Laplacian. Let $B \in \mathbb{R}^{m \times n}$ be the incidence matrix where its row corresponding to edge $e$ is the characteristic (row) vector $\chi_{e}^{T}$. Define $W=\operatorname{diag}\left\{w_{e}\right\}_{e \in E}$ to be the diagonal matrix of edge weights. The Laplacian with respect to graph $G$ is $L_{G} \stackrel{\text { def }}{=} B^{T} W B \in \mathbb{R}^{n \times n}$. It is clear from the definition that $L_{G} \succeq 0$ is PSD and $L_{G}=\sum_{e \in E} L_{e}$. Notice that $\operatorname{ker}\left(L_{G}\right)=\operatorname{ker}\left(W^{1 / 2} B\right)=\operatorname{span}(\mathbb{1})$, and therefore $x^{T} L_{G} x=0$ if and only if $x$ is a constant vector.

Since $L_{G}$ is symmetric, one can diagonalize it and write $L_{G}=\sum_{i=1}^{n-1} \lambda_{i} v_{i} v_{i}^{T}$, where $\lambda_{i}$ 's are the positive eigenvalues of $L_{G}$ and $v_{i}$ 's are the corresponding set of orthogonal eigenvectors. The MoorePenrose pseudoinverse of $L_{G}$ is denoted by $L_{G}^{\dagger} \stackrel{\text { def }}{=} \sum_{i=1}^{n-1} \lambda_{i}^{-1} v_{i} v_{i}^{T}$. For notational convenience, we will stick to $L_{G}^{-1}$ to denote this pseudoinverse, and often use $L_{G}^{-2}$ to denote $\left(L_{G}^{\dagger}\right)^{2}$, and $L_{G}^{-1 / 2}$ to denote $\left(L_{G}^{\dagger}\right)^{1 / 2}$, and so on. We remark here that $L_{G} L_{G}^{-1}=L_{G}^{-1} L_{G}=\sum_{i} v_{i} v_{i}^{T}=I_{\mathrm{im}\left(L_{G}\right)}$. Here, $I_{\mathrm{im}\left(L_{G}\right)}$ is the identity matrix on the image space of $L_{G}$, which is just the space spanned by all the vectors orthogonal to $\mathbb{1}$. For notational convenience, we will often abbreviate $I_{\mathrm{im}\left(L_{G}\right)}$ as $I$.

Throughout this paper, whenever related to graph sparsifications, we denote by

$$
\check{L}_{e} \stackrel{\text { def }}{=} L_{G}^{-1 / 2} L_{e} L_{G}^{-1 / 2} \text { and } \quad \widehat{L}_{e} \stackrel{\text { def }}{=} \frac{L_{G}^{-1 / 2} L_{e} L_{G}^{-1 / 2}}{L_{G}^{-1} \bullet L_{e}}=\frac{\breve{L}_{e}}{L_{G}^{-1} \bullet L_{e}} .
$$

Above, $\breve{L}_{e}$ is the normalized edge Laplacian, and $\widehat{L}_{e}$ is the normalized edge Laplacian scaled by the effective resistance. ( $L_{G}^{-1} \bullet L_{e}$ is the "effective resistance" of the edge $e$, see for instance [SS11]). Both of them have rank 1 , and it satisfies $\operatorname{Tr}\left(\breve{L}_{e}\right) \leq 1$ and $\breve{L}_{e} \preceq I$, while $\operatorname{Tr}\left(\widehat{L}_{e}\right)=1$ and $\widehat{L}_{e} \preceq I$.

It is easy to check from the above definition that $\sum_{e} \breve{L}_{e}=I_{\mathrm{im}\left(L_{G}\right)}$. In addition, letting $w_{e}=$ $L_{G}^{-1} \bullet L_{e}$ be the effective resistence of edge $e$, then $\sum_{e} w_{e} \widehat{L}_{e}=I_{\mathrm{im}\left(L_{G}\right)}$ as well. Notice that $\sum_{e} w_{e}=$ $\operatorname{Tr} I_{\mathrm{im}\left(L_{G}\right)}=n-1$, the dimension of $I_{\mathrm{im}\left(L_{G}\right)}$ (see [SS11]).

From Graph Sparsification to Rank-1 Decomposition Sparsification. As originally shown in [BSS14], one can easily translate the problem of graph spectral sparsification (see Theorem 1) into that of sparsifying sums of rank-1 matrices (see Theorem 2). Indeed, because $I_{\mathrm{im}\left(L_{G}\right)}=\sum_{e \in[m]} \breve{L}_{e}$ is a summation of rank-1 matrices, if one can find scalars $s_{e} \geq 0$ (as per Theorem 2 ) that satisfies $I_{\mathrm{im}\left(L_{G}\right)} \preceq \sum_{e \in[m]} s_{e} \breve{L}_{e} \preceq(1+\varepsilon) I_{\mathrm{im}\left(L_{G}\right)}$, this immediately implies, by the definition of $\breve{L}_{e}$, that $L_{G} \preceq \sum_{e \in[m]} s_{e} L_{e} \preceq(1+\varepsilon) L_{G}$.

\section{Weak Unweighted Sparsifier}

In this section, we consider the weak unweighted spectral sparsification problem very recently studied by Anderson, Gu and Melgaard [AGM14]: for any value $\kappa \in[1, m / n]$, find a $\kappa$-spectral sparsifier of $G$ containing $O(\mathrm{~m} / \kappa)$ distinct edges from $E$, without reweighting. We show that our regret minimization framework allows us to design a simple and almost-quadratic-time algorithm for this problem, improving from the quartic time complexity of [AGM14].

Formally, given any weighted undirected graph $G=(V, E, w)$ with $n$ vertices and $m$ edges, and any value $\kappa \in[1, m / n]$, the task it to find a subset $E_{0} \subseteq E$ containing $O(m / \kappa)$ distinct edges such that

$$
\frac{1}{\kappa} L_{G} \preceq \sum_{e \in E_{0}} L_{e} \preceq L_{G}
$$

This is an unweighted sparsification problem because one is not allowed to reweight the edges in $E_{0}$, in contrast to Theorem 1: and we call it a weak sparsifier because $\kappa$ is usually large. 
Similar to Appendix B. one can easily reduce this graph sparsification problem to sparsifying sums of rank-1 matrices. Given $m$ rank-1 PSD matrices $\breve{L}_{1}, \ldots, \breve{L}_{m} \in \mathbb{R}^{n \times n}$ that satisfies $I=$ $\sum_{e \in[m]} \breve{L}_{e}$, and given some $\kappa \in[1, m / n]$, find a subset $E_{0} \subseteq[m]$ with $O(m / \kappa)$ distinct elements satisfying $\sum_{e \in E_{0}} \breve{L}_{e} \succeq \frac{1}{\kappa} I$.

(In this section, one should feel free to coincide this $\breve{L}_{e}$ with the 'normalized edge Laplacian' introduced in Section B but $\widehat{L}_{e}$ needs not coincide with any graph Laplacian in general.)

We solve this weak unweighted sparsification problem via the following reduction to regret minimization.

If $\kappa \leq 9$, we output $E_{0}=E$ and are done. Otherwise, we choose the $\ell_{1 / 2}$ regularizer and parameter $\alpha=4 \sqrt{n} \kappa$ for MirrorDescent $\ell_{\ell_{1 / 2}}$. At each iteration $k=0,1, \ldots, T-1$, we define $e_{k}=e$ to be the index $e \in[m]$ that maximizes the quantity $\frac{X_{k} \bullet \breve{L}_{e}}{1+X_{k}^{1 / 2} \bullet \alpha \breve{L}_{e}}$ among all edges not chosen before -i.e., all edges in $E \backslash\left\{e_{0}, e_{1}, \ldots, e_{k-1}\right\}$. Next, we feed $F_{k}=\breve{L}_{e_{k}}$ as the feedback matrix to MirrorDescent $\ell_{\ell_{1 / 2}}$ and compute $X_{k+1}$ of the next iteration.

Let us now state a simple property for the selected matrix $\breve{L}_{e_{k}}$ using an averaging argument:

Claim C.1. For each $k=0,1, \ldots, T-1$, we either have $\sum_{j=0}^{k-1} \breve{L}_{e_{j}} \succeq \frac{1}{\kappa} I$ or $\frac{X_{k} \bullet \breve{L}_{e_{k}}}{1+X_{k}^{1 / 2} \bullet \alpha \breve{L}_{e_{k}}} \geq \frac{1}{6 m}$.

Proof. Let us recall that by the definition of MirrorDescent $\ell_{\ell_{1 / 2}}$, we have

$$
X_{k}=\left(\alpha \sum_{j=0}^{k-1} \breve{L}_{e_{j}}-c_{k} I\right)^{-2},
$$

where $c_{k}>0$ is the unique constant that makes $\alpha \sum_{j=0}^{k-1} \breve{L}_{e_{j}} \succ c_{k} I$ and $\operatorname{Tr} X_{k}=1$. Note that if $c_{k} / \alpha \geq \frac{1}{\kappa}$ then we already have $\sum_{j=0}^{k-1} \breve{L}_{e_{j}} \succ \frac{c_{k}}{\alpha} I \succeq \frac{1}{\kappa} I$. Therefore, we can assume $c_{k} / \alpha<\frac{1}{\kappa}$ for the rest of the proof.

One one hand, we have

$$
\begin{aligned}
\sum_{e \notin\left\{e_{0}, \ldots, e_{k-1}\right\}} X_{k} \bullet \breve{L}_{e}=X_{k} \bullet & \left(I-\sum_{j=0}^{k-1} \breve{L}_{e_{j}}\right)=X_{k} \bullet\left(I-\frac{c_{k}}{\alpha} I-\frac{X_{k}^{-1 / 2}}{\alpha}\right) \\
& =\left(1-\frac{c_{k}}{\alpha}\right)-\frac{\operatorname{Tr} X_{k}^{1 / 2}}{\alpha}>1-\frac{1}{\kappa}-\frac{\sqrt{n}}{\alpha}>\frac{5}{6},
\end{aligned}
$$

where the first inequality is due to $\operatorname{Tr} X_{k}^{1 / 2} \leq \sqrt{n}$ and the second inequality is due to our choice of $\alpha=4 \sqrt{n} \kappa$ and the fact that $\kappa>9$.

On the other hand, we have

$$
\begin{aligned}
& \sum_{e \notin\left\{e_{0}, \ldots, e_{k-1}\right\}} \frac{1}{6 m}(\left.1+X_{k}^{1 / 2} \bullet \alpha \breve{L}_{e}\right) \leq \frac{1}{6}+\frac{\alpha}{6 m} X_{k}^{1 / 2} \bullet \sum_{e \notin\left\{e_{0}, \ldots, e_{k-1}\right\}} \alpha \breve{L}_{e} \\
& \leq \frac{1}{6}+\frac{\alpha}{6 m} X_{k}^{1 / 2} \bullet I \leq \frac{1}{6}+\frac{\alpha \sqrt{n}}{6 m}=\frac{1}{6}+\frac{4 n \kappa}{6 m} \leq \frac{5}{6},
\end{aligned}
$$

where the second inequality is because $\sum_{e \notin\left\{e_{0}, \ldots, e_{k-1}\right\}} \breve{L}_{e} \preceq \sum_{e \in[m]} \breve{L}_{e}=I$, the third inequality is because $\operatorname{Tr} X_{k}^{1 / 2} \leq \sqrt{n}$, and the fourth inequality is because $\kappa \leq m / n$.

Combining (C.1) and (C.2), we conclude that there exists at least some index $e \in[\mathrm{m}] \backslash$ $\left\{e_{0}, \ldots, e_{k-1}\right\}$ satisfying that $X_{k} \bullet \breve{L}_{e} \geq \frac{1}{7 m}\left(1+X_{k}^{1 / 2} \bullet \alpha \breve{L}_{e}\right)$, finishing the proof of the claim. 
Now we are ready to apply Theorem 3.2, the regret bound, with our choice of $F_{k}=\breve{L}_{e_{k}}$ :

$$
\begin{aligned}
\forall U \in \Delta_{n \times n}, \quad \sum_{k=0}^{T-1}\left\langle\breve{L}_{e_{k}}, U\right\rangle & \geq \sum_{k=0}^{T-1}\left\langle\breve{L}_{e_{k}}, X_{k}\right\rangle-\alpha \frac{\operatorname{Tr}\left(X_{k} \breve{L}_{e_{k}} X_{k}^{1 / 2} \breve{L}_{e_{k}}\right)}{1+X_{k}^{1 / 2} \bullet \alpha \breve{L}_{e_{k}}}-\frac{2 \sqrt{n}}{\alpha} \\
& =\sum_{k=0}^{T-1}\left\langle\breve{L}_{e_{k}}, X_{k}\right\rangle\left(1-\frac{X_{k}^{1 / 2} \bullet \alpha \breve{L}_{e_{k}}}{1+X_{k}^{1 / 2} \bullet \alpha \check{L}_{e_{k}}}\right)-\frac{2 \sqrt{n}}{\alpha} \\
& =\sum_{k=0}^{T-1} \frac{\breve{L}_{e_{k}} \bullet X_{k}}{1+X_{k}^{1 / 2} \bullet \alpha \breve{L}_{e_{k}}}-\frac{2 \sqrt{n}}{\alpha} .
\end{aligned}
$$

We will now choose $T=9 m / \kappa$. (Notice that $T<m$ because $\kappa>9$.) There are two possibilities according to Claim C.1.

In the first case, we have $\sum_{j=0}^{k-1} \breve{L}_{e_{j}} \succeq \frac{1}{\kappa} I$ for some $k=0,1, \ldots, T-1$ and we are done: that is, defining $E_{0} \stackrel{\text { def }}{=}\left\{e_{0}, e_{1}, \ldots, e_{k-1}\right\}$, we have that $\left|E_{0}\right| \leq T=O(m / \kappa)$ and $I \succeq \sum_{e \in E_{0}} \breve{L}_{e} \succeq \frac{1}{\kappa} I$.

In the second case, we have $\frac{X_{k} \bullet \breve{L}_{e_{k}}}{1+X_{k}^{1 / 2} \bullet \alpha \breve{L}_{e_{k}}} \geq \frac{1}{6 m}$ for all $k=0,1, \ldots, T-1$. Substituting this into (C.3), and choosing $U$ to be the rank 1 matrix corresponding to the smallest eigenvalue of $\sum_{k=0}^{T-1} \breve{L}_{e_{k}}$, we conclude that

$$
\lambda_{\min }\left(\sum_{k=0}^{T-1} \breve{L}_{e_{k}}\right) \geq \sum_{k=0}^{T-1} \frac{1}{6 m}-\frac{1}{2 \kappa}=\frac{1}{\kappa} .
$$

Therefore, defining $E_{0} \stackrel{\text { def }}{=}\left\{e_{0}, e_{1}, \ldots, e_{T-1}\right\}$, we also have $\left|E_{0}\right|=T=O(m / \kappa)$ and $I \succeq \sum_{e \in E_{0}} \breve{L}_{e} \succeq$ $\frac{1}{\kappa} I$. In sum,

Theorem C.2. Given a decomposition $I=\sum_{e \in[m]} \breve{L}_{e}$ of rank-1 PSD matrices, and given some $\kappa \in[1, m / n]$, the above algorithm finds a subset $E_{0} \subseteq[m]$ with $O\left(\frac{m}{\kappa}\right)$ distinct elements satisfying $I \succeq \sum_{e \in E_{0}} \breve{L}_{e} \succeq \frac{1}{\kappa} I$.

We remark here that for graph sparsification, the above algorithm can be implemented to run in time $\widetilde{O}\left(m^{3 / 2} n\right)$, and can be improved to $\widetilde{O}\left(m^{1+1 / q} n\right)$ for any even integer constant $q \geq 2$ if the $\ell_{1-1 / q}$ regularizer is used instead of $\ell_{1 / 2}$. We ignore the implementation details in this version of the paper because it is very similar to the details discussed in Section 6.

\section{Proof of Lemma 2.1}

We state some classical properties for Bregman divergence, which are classical and can be found in for instance [CL06].

Lemma 2.1. The following properties hold for Bregman divergence.

- Non-negativity: $V_{X}(Y) \geq 0$ for all $X, Y \geq 0$.

- The "three-point equality": $\langle\nabla w(X)-\nabla w(Y), X-U\rangle=V_{X}(U)-V_{Y}(U)+V_{Y}(X)$.

- Given $\tilde{X} \succeq 0$ and $X=\arg \min _{Z \in \Delta_{n \times n}} V_{\widetilde{X}}(Z)$ as the Bregman projection, we have the "generalized Pythagorean theorem" for all $U \in \Delta: V_{\widetilde{X}}(U) \geq V_{X}(U)+V_{\widetilde{X}}(X) \geq V_{X}(U)$.

Proof. The non-negativity follows by definition from the convexity of $w(X)$. For every $U \succeq 0$, the "three-point equality" follows from the following inequality.

$$
\begin{aligned}
\langle\nabla w(Y)-\nabla w(Y), Y-U\rangle= & (w(U)-w(Y)-\langle\nabla w(Y), U-Y\rangle)-(w(U)-w(Y)-\langle w(Y), U-Y)\rangle) \\
& -(w(Y)-w(Y)-\langle\nabla w(Y), Y-Y\rangle) \\
= & V_{Y}(U)-V_{Y}(U)-V_{Y}(Y) .
\end{aligned}
$$


For the generalized Pythagorean theorem, we only need to prove $V_{\widetilde{X}}(U) \geq V_{X}(U)+V_{\widetilde{X}}(X)$ because the second inequality follows from the non-negativity of $V_{\widetilde{X}}(X)$. To provide the simplest proof, we only focus on the special case when $w(X)=-\frac{q}{q-1} \operatorname{Tr} X^{1-1 / q}$. (The proof for the entropy regularizer is similar, while the proof for the most general Legendre function case is more involved.)

By definition,

Therefore,

$$
\begin{aligned}
V_{X}(U)+V_{\widetilde{X}}(X)= & X^{-1 / q} \bullet U+\frac{1}{q-1} \operatorname{Tr} X^{1-1 / q}-\frac{q}{q-1} \operatorname{Tr} U^{1-1 / q} \\
& +\widetilde{X}^{-1 / q} \bullet X+\frac{1}{q-1} \operatorname{Tr} \widetilde{X}^{1-1 / q}-\frac{q}{q-1} \operatorname{Tr} X^{1-1 / q} \\
V_{\widetilde{X}}(U)= & \widetilde{X}^{-1 / q} \bullet U+\frac{1}{q-1} \operatorname{Tr} \widetilde{X}^{1-1 / q}-\frac{q}{q-1} \operatorname{Tr} U^{1-1 / q}
\end{aligned}
$$

$$
\begin{aligned}
V_{\widetilde{X}}(U)-\left(V_{X}(U)+V_{\widetilde{X}}(X)\right) & =\widetilde{X}^{-1 / q} \bullet U-X^{-1 / q} \bullet U-\widetilde{X}^{-1 / q} \bullet X+\operatorname{Tr} X^{1-1 / q} \\
& =\left(\widetilde{X}^{-1 / q}-X^{-1 / q}\right) \bullet(U-X) .
\end{aligned}
$$

Since $V_{\widetilde{X}}(U)$ is a convex function and $X=\arg \min _{z \in \Delta} V_{\widetilde{X}}(z)$, for any $U \in \Delta_{n \times n}$ we must have

$$
\left\langle\nabla V_{\widetilde{X}}(X), U-X\right\rangle \geq 0 \Longleftrightarrow\left\langle-X^{-1 / q}+\widetilde{X}^{-1 / q}, U-X\right\rangle \geq 0
$$

This concludes the proof of the lemma.

\section{E $\quad$ Missing Proofs in Section 3}

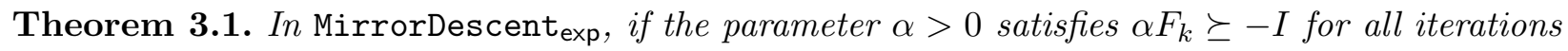
$k=0,1, \ldots, T-1$, then, for every $U \in \Delta_{n \times n}$,

$$
R(U) \stackrel{\text { def }}{=} \sum_{k=0}^{T-1}\left\langle F_{k}, X_{k}-U\right\rangle \leq \alpha \sum_{k=0}^{T-1}\left(X_{k} \bullet\left|F_{k}\right|\right) \cdot\left\|F_{k}\right\|_{\text {spe }}+\frac{V_{X_{0}}(U)}{\alpha} .
$$

We note that $V_{X_{0}}(U) \leq \log n$.

Proof. We prove the theorem by using a two-step description of the mirror descent. For every $k \geq 0$, define $\widetilde{X}_{k+1} \stackrel{\text { def }}{=} \arg \min _{Z \succeq 0}\left\{V_{X_{k}}(Z)+\alpha\left\langle F_{k}, Z\right\rangle\right\}$, where the minimization is over all $Z \succeq 0$, rather than $Z \in \Delta_{n \times n}$. This minimizer $\widetilde{X}_{k+1}$ certainly exists (and equals to $\exp ^{\log X_{k}-\alpha F_{k}}$ ), and it is not hard to verify that $X_{k+1}=\arg \min _{Z \in \Delta_{n \times n}}\left\{V_{\widetilde{X}_{k+1}}(Z)\right\}$. In other words, one can describe the update $X_{k} \rightarrow X_{k+1}$ by adding an intermediate stage $X_{k} \rightarrow \widetilde{X}_{k+1} \rightarrow X_{k+1}$. We also assume that initially we have $\widetilde{X}_{0} \stackrel{\text { def }}{=} X_{0}$.

Noticing that the definition of $\tilde{X}_{k+1}$ implies that $\nabla V_{X_{k}}\left(\widetilde{X}_{k+1}\right)+\alpha F_{k}=0$, which by the definition of $V_{X}(Y)$ is equivalent to $\nabla w\left(X_{k}\right)-\nabla w\left(\widetilde{X}_{k+1}\right)=\alpha F_{k}$. Therefore,

$$
\begin{aligned}
\left\langle\alpha F_{k}, X_{k}-U\right\rangle=\left\langle\nabla w\left(X_{k}\right)-\nabla w\left(\widetilde{X}_{k+1}\right), X_{k}-U\right\rangle & =V_{X_{k}}(U)-V_{\widetilde{X}_{k+1}}(U)+V_{\widetilde{X}_{k+1}}\left(X_{k}\right) \\
& \leq V_{\widetilde{X}_{k}}(U)-V_{\widetilde{X}_{k+1}}(U)+V_{\widetilde{X}_{k+1}}\left(X_{k}\right) .
\end{aligned}
$$

Above, the second equality is due to the three-point equality and the only inequality is due to the generalized Pythagorean theorem of Bregman divergence (see Lemma 2.1). Now,

$$
\begin{aligned}
V_{\widetilde{X}_{k+1}}\left(X_{k}\right) & =X_{k} \bullet\left(\log X_{k}-\log \widetilde{X}_{k+1}\right)+\operatorname{Tr} \widetilde{X}_{k+1}-\operatorname{Tr} X_{k} \\
& =X_{k} \bullet \alpha F_{k}+\operatorname{Tr}\left(e^{\log X_{k}-\alpha F_{k}}\right)-\operatorname{Tr} X_{k} \stackrel{(1)}{\leq} X_{k} \bullet \alpha F_{k}+X_{k} \bullet e^{-\alpha F_{k}}-\operatorname{Tr} X_{k} \\
& \stackrel{(2)}{\leq} X_{k} \bullet \alpha F_{k}+X_{k} \bullet\left(I-\alpha F_{k}+\alpha^{2} F_{k}^{2}\right)-\operatorname{Tr} X_{k}=\alpha^{2} \cdot X_{k} \bullet F_{k}^{2} \stackrel{3}{\leq} \alpha^{2} \cdot\left(X_{k} \bullet\left|F_{k}\right|\right)\left\|F_{k}\right\|_{\text {spe }} .
\end{aligned}
$$


Above, (1) is due to the Golden-Thompson inequality. (2) follows because $e^{-\alpha A} \preceq I-\alpha A+\alpha^{2} A^{2}$, which can be proved after transforming into its eigenbasis, and then using the fact that $e^{-a} \leq$ $1-a+a^{2}$ for all $a \geq-1$. (3) follows because $F_{k}^{2} \preceq\left\|F_{k}\right\|_{\text {spe }} \cdot\left|F_{k}\right|$.

Finally, substituting the above upper bound into (E.1) and telescoping it for $k=0, \ldots, T-1$, we obtain

$$
\sum_{k=0}^{T-1}\left\langle F_{k}, X_{k}-U\right\rangle \leq \frac{V_{\widetilde{X}_{0}}(U)-V_{\widetilde{X}_{T}}(U)}{\alpha}+\alpha \sum_{k=0}^{T-1}\left(X_{k} \bullet\left|F_{k}\right|\right)\left\|F_{k}\right\|_{\text {spe }} .
$$

The desired result of this theorem now follows from the above inequality and the simple upper bound $V_{\widetilde{X}_{0}}(U)=V_{X_{0}}(U) \leq \log n$ and the nonnegativity $V_{\widetilde{X}_{T}}(U) \geq 0$.

Theorem 3.2. In MirrorDescent $\ell_{\ell_{1 / 2}}$ if the parameter $\alpha>0$, and the loss matrix $F_{k}$ is rank one and satisfies $X_{k}^{1 / 2} \bullet \alpha F_{k}>-1$ for all $k$, then, for every $U \in \Delta_{n \times n}$,

$$
R(U) \stackrel{\text { def }}{=} \sum_{k=0}^{T-1}\left\langle F_{k}, X_{k}-U\right\rangle \leq \alpha \cdot \sum_{k=0}^{T-1} \frac{\left(X_{k} \bullet F_{k}\right)\left(X_{k}^{1 / 2} \bullet F_{k}\right)}{1+X_{k}^{1 / 2} \bullet \alpha F_{k}}+\frac{V_{X_{0}}(U)}{\alpha}
$$

If we instead have $X_{k}^{1 / 2} \bullet \alpha F_{k} \geq-\frac{1}{2}$, the above bound can be simplified as

$$
R(U) \stackrel{\text { def }}{=} \sum_{k=0}^{T-1}\left\langle F_{k}, X_{k}-U\right\rangle \leq 2 \alpha \cdot \sum_{k=0}^{T-1}\left(X_{k} \bullet F_{k}\right)\left(X_{k}^{1 / 2} \bullet F_{k}\right)+\frac{V_{X_{0}}(U)}{\alpha} .
$$

We note that $V_{X_{0}}(U) \leq 2 \sqrt{n}$.

Proof. We prove the theorem by using a two-step description of the mirror descent. For every $k \geq 0$, define $\widetilde{X}_{k+1} \stackrel{\text { def }}{=} \arg \min _{Z \succeq 0}\left\{V_{X_{k}}(Z)+\alpha\left\langle F_{k}, Z\right\rangle\right\}$, where the minimization is over all $Z \succeq 0$, rather than $Z \in \Delta_{n \times n}$. We claim that this minimizer $\widetilde{X}_{k+1}$ exists and is strictly positive definite, because one can choose $Z=\widetilde{X}_{k+1}=\left(X_{k}^{-1 / 2}+\alpha F_{k}\right)^{-2} \succ 0$ to make the gradient zero:

$$
\nabla V_{X_{k}}\left(\widetilde{X}_{k+1}\right)+\alpha F_{k}=\nabla w\left(\widetilde{X}_{k+1}\right)-\nabla w\left(X_{k}\right)+\alpha F_{k}=-\widetilde{X}_{k+1}^{-1 / 2}+X_{k}^{-1 / 2}+\alpha F_{k}=0 .
$$

This uses our assumption $X_{k}^{1 / 2} \bullet \alpha F_{k}>-1$ which is equivalent to $\alpha F_{k} \succ-X_{k}^{-1 / 2}, 8$ so as to ensure that $\tilde{X}_{k+1}$ is well defined.

Next, it is easy to verify that $X_{k+1}=\arg \min _{Z \in \Delta_{n \times n}}\left\{V_{\widetilde{X}_{k+1}}(Z)\right\}$. In other words, one can describe the update $X_{k} \rightarrow X_{k+1}$ by adding an intermediate stage $X_{k} \rightarrow \widetilde{X}_{k+1} \rightarrow X_{k+1}$. We assume for notational simplicity that $\widetilde{X}_{0} \stackrel{\text { def }}{=} X_{0}$.

Using (E.2), we easily obtain that

$$
\begin{aligned}
\left\langle\alpha F_{k}, X_{k}-U\right\rangle=\left\langle\nabla w\left(X_{k}\right)-\nabla w\left(\widetilde{X}_{k+1}\right), X_{k}-U\right\rangle & =V_{X_{k}}(U)-V_{\widetilde{X}_{k+1}}(U)+V_{\widetilde{X}_{k+1}}\left(X_{k}\right) \\
& \leq V_{\widetilde{X}_{k}}(U)-V_{\widetilde{X}_{k+1}}(U)+V_{\widetilde{X}_{k+1}}\left(X_{k}\right) .
\end{aligned}
$$

Above, the second equality is due to the three-point equality and the only inequality is due to the generalized Pythagorean theorem of Bregman divergence (see Lemma 2.1).

We now exactly compute $V_{\widetilde{X}_{k+1}}\left(X_{k}\right)$ in two cases.

- If $\alpha F_{k}=-u u^{T}$ is negative semidefinite, using the Sherman-Morrison formula,

$$
\operatorname{Tr} \widetilde{X}_{k+1}^{1 / 2}=\operatorname{Tr}\left(\left(X_{k}^{-1 / 2}-u u^{T}\right)^{-1}\right)=\operatorname{Tr}\left(X_{k}^{1 / 2}+\frac{X_{k}^{1 / 2} u u^{T} X_{k}^{1 / 2}}{1-u^{T} X_{k}^{1 / 2} u}\right) .
$$

\footnotetext{
${ }^{8}$ This is because, if $F_{k}=-u u^{T}$, then $X_{k}^{1 / 2} \bullet\left(-\alpha u u^{T}\right)>-1$ is equivalent to $\alpha u^{T} X_{k}^{1 / 2} u<1$, which is further equivalent to $\alpha \operatorname{Tr} X_{k}^{1 / 4} u u^{T} X_{k}^{1 / 4}<1$. However, since $X_{k}^{1 / 4} u u^{T} X_{k}^{1 / 4}$ is a rank-1 matrix, this is finally equivalent to $\alpha u u^{T} \prec X_{k}^{-1 / 2}$.
} 
Therefore,

$$
\begin{aligned}
V_{\widetilde{X}_{k+1}}\left(X_{k}\right) & =\widetilde{X}_{k+1}^{-1 / 2} \bullet X_{k}+\operatorname{Tr} \widetilde{X}_{k+1}^{1 / 2}-2 \operatorname{Tr} X_{k}^{1 / 2}=\left(X_{k}^{-1 / 2}-u u^{T}\right) \bullet X_{k}+\operatorname{Tr} \widetilde{X}_{k+1}^{1 / 2}-2 \operatorname{Tr} X_{k}^{1 / 2} \\
& =-u u^{T} \bullet X_{k}+\left(\operatorname{Tr} \widetilde{X}_{k+1}^{1 / 2}-\operatorname{Tr} X_{k}^{1 / 2}\right)=-u^{T} X_{k} u+\frac{u^{T} X_{k} u}{1-u^{T} X_{k}^{1 / 2} u} \\
& =\frac{u^{T} X_{k} u \cdot u^{T} X_{k}^{1 / 2} u}{1-u^{T} X_{k}^{1 / 2} u}=\alpha^{2} \frac{\left(X_{k} \bullet F_{k}\right)\left(X_{k}^{1 / 2} \bullet F_{k}\right)}{1+X_{k}^{1 / 2} \bullet \alpha F_{k}} .
\end{aligned}
$$

- If $\alpha F_{k}=u u^{T}$ is positive semidefinite, using the Sherman-Morrison formula,

$$
\operatorname{Tr} \widetilde{X}_{k+1}^{1 / 2}=\operatorname{Tr}\left(\left(X_{k}^{-1 / 2}+u u^{T}\right)^{-1}\right)=\operatorname{Tr}\left(X_{k}^{1 / 2}-\frac{X_{k}^{1 / 2} u u^{T} X_{k}^{1 / 2}}{1+u^{T} X_{k}^{1 / 2} u}\right) .
$$

Therefore,

$$
\begin{aligned}
V_{\widetilde{X}_{k+1}}\left(X_{k}\right) & =\widetilde{X}_{k+1}^{-1 / 2} \bullet X_{k}+\operatorname{Tr} \widetilde{X}_{k+1}^{1 / 2}-2 \operatorname{Tr} X_{k}^{1 / 2}=\left(X_{k}^{-1 / 2}+u u^{T}\right) \bullet X_{k}+\operatorname{Tr} \widetilde{X}_{k+1}^{1 / 2}-2 \operatorname{Tr} X_{k}^{1 / 2} \\
& =u u^{T} \bullet X_{k}+\left(\operatorname{Tr} \widetilde{X}_{k+1}^{1 / 2}-\operatorname{Tr} X_{k}^{1 / 2}\right)=u^{T} X_{k} u+\frac{u^{T} X_{k} u}{1+u^{T} X_{k}^{1 / 2} u} \\
& =\frac{u^{T} X_{k} u \cdot u^{T} X_{k}^{1 / 2} u}{1+u^{T} X_{k}^{1 / 2} u}=\alpha^{2} \frac{\left(X_{k} \bullet F_{k}\right)\left(X_{k}^{1 / 2} \bullet F_{k}\right)}{1+X_{k}^{1 / 2} \bullet \alpha F_{k}} .
\end{aligned}
$$

Finally, substituting the above computation of $V_{\widetilde{X}_{k+1}}\left(X_{k}\right)$ into (E.3) and telescoping it for $k=$ $0, \ldots, T-1$, we obtain

$$
\sum_{k=0}^{T-1}\left\langle F_{k}, X_{k}-U\right\rangle \leq \frac{V_{\widetilde{X}_{0}}(U)-V_{\widetilde{X}_{T}}(U)}{\alpha}+\alpha \sum_{k=0}^{T-1} \frac{\left(X_{k} \bullet F_{k}\right)\left(X_{k}^{1 / 2} \bullet F_{k}\right)}{1+X_{k}^{1 / 2} \bullet \alpha F_{k}} .
$$

The desired result of this theorem now follows from the above inequality and the simple upper bound $V_{\widetilde{X}_{0}}(U)=V_{X_{0}}(U) \leq 2 \sqrt{n}$ and the nonnegativity $V_{\widetilde{X}_{T}}(U) \geq 0$.

The next theorem generalizes Theorem 3.2 to high rank loss matrices and $\ell_{1-1 / q}$-regularizers with $q \geq 2$. The key idea is to replace the use of the Sherman-Morrison formula in the proof of Theorem 3.2 with the Woodbury formula so as to allow $F_{k}$ to be of high rank. It also uses the Lieb-Thirring trace inequality to handle arbitrary $q \geq 2$.

Theorem 3.3. In MirrorDescent $\ell_{\ell_{1-1}}$ with $q \geq 2$ and $\alpha>0$, if the loss matrix $F_{k}$ is either positive or negative semidefinite and satisfies $\alpha X_{k}^{1 / 2 q} F_{k} X_{k}^{1 / 2 q} \succeq-\frac{1}{2 q} I$ for all $k$, then,

$$
\forall U \in \Delta_{n \times n}, \quad R(U) \stackrel{\text { def }}{=} \sum_{k=0}^{T-1}\left\langle F_{k}, X_{k}-U\right\rangle \leq O(q \alpha) \sum_{k=0}^{T-1}\left(X_{k} \bullet\left|F_{k}\right|\right) \cdot\left\|X_{k}^{1 / 2 q} F_{k} X_{k}^{1 / 2 q}\right\|_{\text {spe }}+\frac{V_{X_{0}}(U)}{\alpha} .
$$

We note that $V_{X_{0}}(U) \leq \frac{q}{q-1} n^{1 / q}$.

Proof. We prove the theorem by using a two-step description of the mirror descent. For every $k \geq 0$, define $\widetilde{X}_{k+1} \stackrel{\text { def }}{=} \arg \min _{Z \succeq 0}\left\{V_{X_{k}}(Z)+\alpha\left\langle F_{k}, Z\right\rangle\right\}$, where the minimization is over all $Z \succeq 0$, rather than $Z \in \Delta_{n \times n}$. We claim that this minimizer $\widetilde{X}_{k+1}$ exists and is strictly positive definite, because one can choose $Z=\widetilde{X}_{k+1}=\left(X_{k}^{-1 / q}+\alpha F_{k}\right)^{-q} \succ 0$ to make the gradient zero:

$$
\nabla V_{X_{k}}\left(\tilde{X}_{k+1}\right)+\alpha F_{k}=\nabla w\left(\tilde{X}_{k+1}\right)-\nabla w\left(X_{k}\right)+\alpha F_{k}=-\tilde{X}_{k+1}^{-1 / q}+X_{k}^{-1 / q}+\alpha F_{k}=0 .
$$


This uses our assumption $\alpha X_{k}^{1 / 2 q} F_{k} X_{k}^{1 / 2 q} \succeq-\frac{1}{2 q} I$ which certainly implies $\alpha F_{k, i} \succeq-\frac{1}{2} X_{k}^{-1 / q}$, so as to ensure that $\widetilde{X}_{k+1}$ is well defined.

Next, it is easy to verify that $X_{k+1}=\arg \min _{Z \in \Delta}\left\{V_{\widetilde{X}_{k+1}}(Z)\right\}$. In other words, one can describe the update $X_{k} \rightarrow X_{k+1}$ by adding an intermediate stage $X_{k} \rightarrow \tilde{X}_{k+1} \rightarrow X_{k+1}$. We assume for notational simplicity that $\widetilde{X}_{0} \stackrel{\text { def }}{=} X_{0}$.

Using (E.4), we easily obtain that

$$
\begin{aligned}
\left\langle\alpha F_{k}, X_{k}-U\right\rangle=\left\langle\nabla w\left(X_{k}\right)-\nabla w\left(\widetilde{X}_{k+1}\right), X_{k}-U\right\rangle & =V_{X_{k}}(U)-V_{\widetilde{X}_{k+1}}(U)+V_{\widetilde{X}_{k+1}}\left(X_{k}\right) \\
& \leq V_{\widetilde{X}_{k}}(U)-V_{\widetilde{X}_{k+1}}(U)+V_{\widetilde{X}_{k+1}}\left(X_{k}\right) .
\end{aligned}
$$

Above, the second equality is due to the three-point equality and the only inequality is due to the generalized Pythagorean theorem of Bregman divergence (see Lemma 2.1).

We now upper bound $V_{\widetilde{X}_{k+1}}\left(X_{k}\right)$ in two cases: the case when $\alpha F_{k}=-P P^{T} \preceq 0$ and the case when $\alpha F_{k}=P P^{T} \succeq 0$. In both cases, we denote by $\beta \stackrel{\text { def }}{=} \alpha\left\|X_{k}^{1 / 2 q} F_{k} X_{k}^{1 / 2 q}\right\|_{\text {spe }}=\left\|X_{k}^{1 / 2 q} P P^{T} X_{k}^{1 / 2 q}\right\|_{\text {spe }}$. Notice that this implies 9

$$
X_{k}^{1 / 2 q} P P^{T} X_{k}^{1 / 2 q} \preceq \beta I \quad \text { and } \quad P^{T} X_{k}^{1 / q} P \preceq \beta I .
$$

- If $\alpha F_{k}=-P P^{T}$, we have $X_{k}^{-1 / q} \succ P P^{T}$ and $\beta \leq \frac{1}{2 q}$ by our assumption, so using the Sherman-Morrison-Woodbury formula,

$$
\begin{aligned}
\operatorname{Tr} \widetilde{X}_{k+1}^{1-1 / q}=\operatorname{Tr}\left(\left(X_{k}^{-1 / q}-P P^{T}\right)^{-1}\right)^{q-1} & =\operatorname{Tr}\left(X_{k}^{1 / q}+X_{k}^{1 / q} P\left(I-P^{T} X_{k}^{1 / q} P\right)^{-1} P^{T} X_{k}^{1 / q}\right)^{q-1} \\
& \leq \operatorname{Tr}\left(X_{k}^{1 / q}+\frac{X_{k}^{1 / q} P P^{T} X_{k}^{1 / q}}{1-\beta}\right)^{q-1}
\end{aligned}
$$

where the last inequality follows because $\left(I-P^{T} X_{k}^{1 / q} P\right)^{-1} \preceq \frac{1}{1-\beta} I$ owing to (E.6), as well as $A \preceq B \Longrightarrow \operatorname{Tr} A^{n} \leq \operatorname{Tr} B^{n}$. We continue and write

$$
\begin{aligned}
\operatorname{Tr} \widetilde{X}_{k+1}^{1-1 / q} \leq \operatorname{Tr}\left(X_{k}^{1 / q}+\frac{X_{k}^{1 / q} P P^{T} X_{k}^{1 / q}}{1-\beta}\right)^{q-1} & =\operatorname{Tr}\left(X_{k}^{1 / 2 q}\left(I+\frac{X_{k}^{1 / 2 q} P P^{T} X_{k}^{1 / 2 q}}{1-\beta}\right) X_{k}^{1 / 2 q}\right)^{q-1} \\
& \leq \operatorname{Tr}\left(X_{k}^{(q-1) / 2 q}\left(I+\frac{X_{k}^{1 / 2 q} P P^{T} X_{k}^{1 / 2 q}}{1-\beta}\right)^{q-1} X_{k}^{(q-1) / 2 q}\right) \\
& =\operatorname{Tr}\left(X_{k}^{1-1 / q}\left(I+\frac{X_{k}^{1 / 2 q} P P^{T} X_{k}^{1 / 2 q}}{1-\beta}\right)^{q-1}\right),
\end{aligned}
$$

where the inequality uses the Lieb-Thirring trace inequality (which relies on the fact that $q-1 \geq 1$ ). Finally, denoting by $D \stackrel{\text { def }}{=} \frac{X_{k}^{1 / 2 q} P P^{T} X_{k}^{1 / 2 q}}{1-\beta} \preceq \frac{\beta}{1-\beta} I$ (which uses (E.6) again), we have

$$
(I+D)^{q-1} \preceq I+(q-1) D+O\left(q^{2} \beta\right) \cdot D .
$$

This matrix inequality can be proved by first turning into its eigenbasis, and then verifying that $(1+x)^{q-1} \leq 1+(q-1) x+O\left(q^{2} \beta\right) x$ for all $x \in\left[0, \frac{\beta}{1-\beta}\right]$ (which uses the fact that $\left.\beta \leq 1 / 2 q\right)$.

\footnotetext{
${ }^{9}$ The second inequality is because $P^{T} X_{k}^{1 / q} P=\left(P^{T} X_{k}^{1 / 2 q}\right)\left(P^{T} X_{k}^{1 / 2 q}\right)^{T}$ and has the same largest eigenvalue as $\left(P^{T} X_{k}^{1 / 2 q}\right)^{T}\left(P^{T} X_{k}^{1 / 2 q}\right)=X_{k}^{1 / 2 q} P P^{T} X_{k}^{1 / 2 q}$.
} 
Using this inequality, we conclude that

$$
\begin{aligned}
\operatorname{Tr} \widetilde{X}_{k+1}^{1-1 / q} & \leq \operatorname{Tr}\left(X_{k}^{1-1 / q}\left(I+\frac{X_{k}^{1 / 2 q} P P^{T} X_{k}^{1 / 2 q}}{1-\beta}\right)^{q-1}\right) \\
& \leq \operatorname{Tr}\left(X_{k}^{1-1 / q}\left(I+\left((q-1)+O\left(q^{2} \beta\right)\right) \frac{X_{k}^{1 / 2 q} P P^{T} X_{k}^{1 / 2 q}}{1-\beta}\right)\right) \\
& =\operatorname{Tr} X_{k}^{1-1 / q}+(q-1)(1+O(q \beta)) X_{k} \bullet P P^{T} .
\end{aligned}
$$

Therefore,

$$
\begin{aligned}
V_{\widetilde{X}_{k+1}}\left(X_{k}\right) & =\widetilde{X}_{k+1}^{-1 / q} \bullet X_{k}+\frac{1}{q-1} \operatorname{Tr} \widetilde{X}_{k+1}^{1-1 / q}-\frac{q}{q-1} \operatorname{Tr} X_{k}^{1-1 / q} \\
& =\left(X_{k}^{-1 / q}-P P^{T}\right) \bullet X_{k}+\frac{1}{q-1} \operatorname{Tr} \widetilde{X}_{k+1}^{1-1 / q}-\frac{q}{q-1} \operatorname{Tr} X_{k}^{1-1 / q} \\
& =-P P^{T} \bullet X_{k}+\frac{1}{q-1}\left(\operatorname{Tr} \widetilde{X}_{k+1}^{1-1 / q}-\operatorname{Tr} X_{k}^{1-1 / q}\right) \\
& =O(q \beta) \cdot P P^{T} \bullet X_{k}=O\left(q \alpha^{2}\right)\left(X_{k} \bullet\left|F_{k}\right|\right) \cdot\left\|X_{k}^{1 / 2 q} F_{k} X_{k}^{1 / 2 q}\right\|_{\text {spe }}
\end{aligned}
$$

- If $\alpha F_{k}=P P^{T}$, using the Sherman-Morrison-Woodbury formula,

$$
\begin{aligned}
\operatorname{Tr} \widetilde{X}_{k+1}^{1-1 / q}=\operatorname{Tr}\left(\left(X_{k}^{-1 / q}+P P^{T}\right)^{-1}\right)^{q-1} & =\operatorname{Tr}\left(X_{k}^{1 / q}-X_{k}^{1 / q} P\left(I+P^{T} X_{k}^{1 / q} P\right)^{-1} P^{T} X_{k}^{1 / q}\right)^{q-1} \\
& \leq \operatorname{Tr}\left(X_{k}^{1 / q}-\frac{X_{k}^{1 / q} P P^{T} X_{k}^{1 / q}}{1+\beta}\right)^{q-1}
\end{aligned}
$$

where the last inequality follows because $\left(I+P^{T} X_{k}^{1 / q} P\right)^{-1} \succeq \frac{1}{1+\beta} I$ owing to (E.6), as well as $A \preceq B \Longrightarrow \operatorname{Tr} A^{n} \leq \operatorname{Tr} B^{n}$. We continue and write

$$
\begin{aligned}
\operatorname{Tr} \widetilde{X}_{k+1}^{1-1 / q} \leq \operatorname{Tr}\left(X_{k}^{1 / q}-\frac{X_{k}^{1 / q} P P^{T} X_{k}^{1 / q}}{1+\beta}\right)^{q-1} & =\operatorname{Tr}\left(X_{k}^{1 / 2 q}\left(I-\frac{X_{k}^{1 / 2 q} P P^{T} X_{k}^{1 / 2 q}}{1+\beta}\right) X_{k}^{1 / 2 q}\right)^{q-1} \\
& \leq \operatorname{Tr}\left(X_{k}^{(q-1) / 2 q}\left(I-\frac{X_{k}^{1 / 2 q} P P^{T} X_{k}^{1 / 2 q}}{1+\beta}\right)^{q-1} X_{k}^{(q-1) / 2 q}\right) \\
& =\operatorname{Tr}\left(X_{k}^{1-1 / q}\left(I-\frac{X_{k}^{1 / 2 q} P P^{T} X_{k}^{1 / 2 q}}{1+\beta}\right)^{q-1}\right),
\end{aligned}
$$

where the inequality again uses the Lieb-Thirring trace inequality. Denoting by $D \stackrel{\text { def }}{=} \frac{X_{k}^{1 / 2 q} P P^{T} X_{k}^{1 / 2 q}}{1+\beta} \preceq$ $\frac{\beta}{1+\beta} I$ (which uses (E.6) again), we see that

$$
(I-D)^{q-1} \preceq I-(q-1) D+O\left(q^{2} \beta\right) \cdot D .
$$

This matrix inequality can be proved by first turning into its eigenbasis, and then verifying that $(1-x)^{q-1} \leq 1-(q-1) x+O\left(q^{2} \beta\right) x$ for all $x \in\left[0, \frac{\beta}{1+\beta}\right]$ (which uses the fact that $\beta \leq 1 / 2 q$ ). This concludes that

$$
\begin{aligned}
\operatorname{Tr} \widetilde{X}_{k+1}^{1-1 / q} & \leq \operatorname{Tr}\left(X_{k}^{1-1 / q}\left(I-\frac{X_{k}^{1 / 2 q} P P^{T} X_{k}^{1 / 2 q}}{1+\beta}\right)^{q-1}\right) \\
& \leq \operatorname{Tr}\left(X_{k}^{1-1 / q}\left(I-(q-1)(1-O(q \beta)) \frac{X_{k}^{1 / 2 q} P P^{T} X_{k}^{1 / 2 q}}{1+\beta}\right)\right) \\
& =\operatorname{Tr} X_{k}^{1-1 / q}-(q-1)(1-O(q \beta)) X_{k} \bullet P P^{T} .
\end{aligned}
$$


Therefore,

$$
\begin{aligned}
V_{\widetilde{X}_{k+1}}\left(X_{k}\right) & =\widetilde{X}_{k+1}^{-1 / q} \bullet X_{k}+\frac{1}{q-1} \operatorname{Tr} \widetilde{X}_{k+1}^{1-1 / q}-\frac{q}{q-1} \operatorname{Tr} X_{k}^{1-1 / q} \\
& =\left(X_{k}^{-1 / q}+P P^{T}\right) \bullet X_{k}+\frac{1}{q-1} \operatorname{Tr} \widetilde{X}_{k+1}^{1-1 / q}-\frac{q}{q-1} \operatorname{Tr} X_{k}^{1-1 / q} \\
& =P P^{T} \bullet X_{k}+\frac{1}{q-1}\left(\operatorname{Tr} \widetilde{X}_{k+1}^{1-1 / q}-\operatorname{Tr} X_{k}^{1-1 / q}\right) \\
& =O(q \beta) \cdot P P^{T} \bullet X_{k}=O\left(q \alpha^{2}\right)\left(X_{k} \bullet\left|F_{k}\right|\right) \cdot\left\|X_{k}^{1 / 2 q} F_{k} X_{k}^{1 / 2 q}\right\|_{\text {spe }} .
\end{aligned}
$$

Finally, substituting the above upper bound on $V_{\widetilde{X}_{k+1}}\left(X_{k}\right)$ into (E.5) and telescoping it for $k=$ $0, \ldots, T-1$, we obtain

$$
\sum_{k=0}^{T-1}\left\langle F_{k}, X_{k}-U\right\rangle \leq \frac{V_{\widetilde{X}_{0}}(U)-V_{\widetilde{X}_{T}}(U)}{\alpha}+O(q \alpha) \sum_{k=0}^{T-1}\left(X_{k} \bullet\left|F_{k}\right|\right) \cdot\left\|X_{k}^{1 / 2 q} F_{k} X_{k}^{1 / 2 q}\right\|_{\text {spe }} .
$$

The desired result of this theorem now follows from the above inequality and the simple upper bound $V_{\widetilde{X}_{0}}(U)=V_{X_{0}}(U) \leq \frac{q}{q-1} n^{1 / q}$ and the nonnegativity $V_{\widetilde{X}_{T}}(U) \geq 0$.

\section{F Robust Linear-Sized Sparsification}

In this section, we deduce the more generalized version of the same result presented in Section 5. with the following major differences.

- Regularizer. In this section, we allow the general $\ell_{1-1 / q}$ regularizer to be used, for any even integer $q \geq 2$, rather than just the $\ell_{1 / 2}$ regularizer. (The assumption on $q$ being even integer rather than all reals no less than 2 is only for the sake of proof convenience.)

- High rank. In this section, we allow $\widehat{L}_{e}$ to be possibly of high rank, rather than just rank 1 .

- Approximate computations. In this section, we allow many computations to be approximate rather than exact. This will enable the algorithm to be more efficiently implemented in the next section (Appendix G). In particular, we allow the following quantities to be approximately computed.

- We only need $\operatorname{Tr} \widehat{L}_{e}$ to be in $\left[1-\varepsilon_{1}, 1\right]$ rather than exactly one.

- We only need $\operatorname{Tr} X_{k}$ and $\operatorname{Tr} Y_{k}$ to be in $\left[1,1+\varepsilon_{1}\right]$ rather than exactly one.

- We only need $\widehat{L}_{e} \bullet X_{k}$ and $\widehat{L}_{e} \bullet Y_{k}$ to be computed only up to a $\left(1+\varepsilon_{1}\right)$ multiplicative error.

We will assume throughout this paper that $\varepsilon_{1}<1 / 2$.

\section{F.1 The Problem}

Suppose we are given a decomposition of the identity matrix $I=\sum_{e=1}^{m} w_{e} \widehat{L}_{e}$, where each $\widehat{L}_{e}$ satisfies (1) $0 \preceq \widehat{L}_{e} \preceq I$, (2) $\operatorname{Tr} \widehat{L}_{e} \in\left[1-\varepsilon_{1}, 1\right]$, and (3) $\widehat{L}_{e}$ may be of high rank. The weights $w_{e}>0$ may be unknown.

In this section, we are interested in using the $\ell_{1-1 / q}$ regularizer for MirrorDescent $\ell_{\ell_{1-1 / q}}$ in order to find scalars $s_{e} \geq 0$ satisfying

$$
I \preceq \sum_{e=1}^{m} s_{e} \cdot \widehat{L}_{e} \preceq\left(1+\sqrt{\frac{8 q^{2}}{q-1}} \cdot \varepsilon+O\left(\varepsilon_{1}+q \varepsilon^{2}+\varepsilon_{1} \varepsilon \sqrt{q}\right)\right) I
$$

while the sparsity of $s$-that is, $\left|\left\{e \in[m]: s_{e}>0\right\}\right|$ - is at most $n / \varepsilon^{2}$. We will not worry about the running time in this section, and defer all the implementation details to Appendix G. 
Throughout this section, we pick $w(X)$ to be the $\ell_{1-1 / q}$ regularizer and $V_{X}(Y)$ to be its induced Bregman divergence.

\section{F.2 Our Algorithm}

Maintain two sequences $X_{k}, Y_{k} \succeq 0$ satisfying $\operatorname{Tr} X_{k}, \operatorname{Tr} Y_{k} \in\left[1,1+\varepsilon_{1}\right]$. At the very beginning we choose $X_{0}=\frac{1}{n} I$ and $Y_{0}=\frac{1}{n} I$ as before.

At each iteration $k=0,1, \ldots, T-1$, find an arbitrary $e_{k}$ such that

$$
\operatorname{Dot}\left(\widehat{L}_{e_{k}}, X_{k}\right) \leq\left(1+\varepsilon_{1}\right)^{2} \operatorname{Dot}\left(\widehat{L}_{e_{k}}, Y_{k}\right) \text {, }
$$

where $\operatorname{Dot}\left(\widehat{L}_{e}, X\right)$ is some algorithm ${ }^{10}$ that approximately computes $\widehat{L}_{e} \bullet X$ and satisfies

$$
\widehat{L}_{e} \bullet X \leq \operatorname{Dot}\left(\widehat{L}_{e}, X\right) \leq\left(1+\varepsilon_{1}\right) \cdot \widehat{L}_{e} \bullet X \text {. }
$$

We can always do so because after averaging,

$$
\begin{aligned}
\sum_{e} w_{e} \operatorname{Dot}\left(\widehat{L}_{e}, X_{k}\right) & \leq\left(1+\varepsilon_{1}\right) \sum_{e}\left(w_{e} \widehat{L}_{e}\right) \bullet X_{k}=\left(1+\varepsilon_{1}\right) \operatorname{Tr} X_{k} \\
& \leq\left(1+\varepsilon_{1}\right)^{2} \operatorname{Tr} Y_{k}=\left(1+\varepsilon_{1}\right)^{2} \sum_{e}\left(w_{e} \widehat{L}_{e}\right) \bullet Y_{k} \leq\left(1+\varepsilon_{1}\right)^{2} \sum_{e} w_{e} \operatorname{Dot}\left(\widehat{L}_{e}, Y_{k}\right) .
\end{aligned}
$$

At each iteration $k=0,1, \ldots, T-1$, we perform updates by finding, ${ }^{11}$ arbitrary $\delta_{X}, \delta_{Y} \geq 0$ satisfying

$$
Y_{k}^{-1 / q}+\frac{\alpha \widehat{L}_{e_{k}}}{\operatorname{Dot}\left(\widehat{L}_{e_{k}}, Y_{k}\right)^{1 / q}}-\delta_{Y} I \succeq 0 \quad \text { and } \quad \operatorname{Tr} X_{k+1}, \operatorname{Tr} Y_{k+1} \in\left[1,1+\varepsilon_{1}\right],
$$

where

$X_{k+1} \stackrel{\text { def }}{=}\left(X_{k}^{-1 / q}+\frac{-\alpha \widehat{L}_{e_{k}}}{\operatorname{Dot}\left(\widehat{L}_{e_{k}}, X_{k}\right)^{1 / q}}+\delta_{X} I\right)^{-q} \quad$ and $\quad Y_{k+1} \stackrel{\text { def }}{=}\left(Y_{k}^{-1 / q}+\frac{\alpha \widehat{L}_{e_{k}}}{\operatorname{Dot}\left(\widehat{L}_{e_{k}}, Y_{k}\right)^{1 / q}}-\delta_{Y} I\right)^{-q}$. Above, $\alpha>0$ is some parameter that will be specified at the end of this section. Note that this corresponds to performing updates

$$
\begin{aligned}
& \text { " } X_{k+1} \leftarrow \underset{Z \in \Delta_{n \times n}}{\arg \min }\left\{V_{X_{k}}(Z)+\left\langle\frac{-\alpha \widehat{L}_{e_{k}}}{\operatorname{Dot}\left(\widehat{L}_{e_{k}}, X_{k}\right)^{1 / q}}, Z\right\rangle\right\} \quad \text { " and } \\
& \text { " } Y_{k+1} \leftarrow \underset{Z \in \Delta_{n \times n}}{\arg \min }\left\{V_{Y_{k}}(Z)+\left\langle\frac{\alpha \widehat{L}_{e_{k}}}{\operatorname{Dot}\left(\widehat{L}_{e_{k}}, Y_{k}\right)^{1 / q}}, Z\right\rangle\right\} \quad "
\end{aligned}
$$

however, we have not required $\operatorname{Tr} X_{k+1}=\operatorname{Tr} Y_{k+1}$ to be precisely equal to 1 .

For analysis purpose only, we also define $\widetilde{X}_{k+1}$ and $\widetilde{Y}_{k+1}$ to be similar updates but without $\delta_{X}$ or $\delta_{Y}$ :

$$
\widetilde{X}_{k+1} \stackrel{\text { def }}{=}\left(X_{k}^{-1 / q}+\frac{-\alpha \widehat{L}_{e_{k}}}{\operatorname{Dot}\left(\widehat{L}_{e_{k}}, X_{k}\right)^{1 / q}}\right)^{-q} \text { and } \quad \widetilde{Y}_{k+1} \stackrel{\text { def }}{=}\left(Y_{k}^{-1 / q}+\frac{\alpha \widehat{L}_{e_{k}}}{\operatorname{Dot}\left(\widehat{L}_{e_{k}}, Y_{k}\right)^{1 / q}}\right)^{-q} .
$$

We assume also $\widetilde{X}_{0} \stackrel{\text { def }}{=} X_{0}$.

Note that $\widetilde{Y}_{k+1}$ is always well defined. Claim F.1 below shows that as long as $\alpha<1$, it always satisfies $X_{k}^{-1 / q}+\frac{-\alpha \widehat{L}_{e_{k}}}{\operatorname{Dot}\left(\widehat{L}_{e_{k}}, X_{k}\right)^{1 / q}} \succeq 0$, so $\widetilde{X}_{k+1}$ is also well defined.

Claim F.1. For every $e \in[m]$, we have $X_{k}^{-1 / q} \succeq \frac{\widehat{L}_{e}}{\left(\widehat{L}_{e} \bullet X_{k}\right)^{1 / q}} \succeq \frac{\widehat{L}_{e}}{\operatorname{Dot}\left(\widehat{L}_{e}, X_{k}\right)^{1 / q}}$. In addition, denoting by $\frac{\alpha \widehat{L}_{e}}{\operatorname{Dot}\left(\widehat{L}_{e}, X_{k}\right)^{1 / q}}=P P^{T}$, we have $0 \preceq P^{T} X_{k}^{1 / q} P \preceq \alpha I$.

\footnotetext{
${ }^{10}$ The implementation of this algorithm will be described in Appendix G.

${ }^{11}$ The existence of such $\delta_{X}$ and $\delta_{Y}$ shall become soon (due to Claim F.1). The implementation of these updates will be described in Appendix G.
} 
Similarly, for every $e \in[m]$, we have $Y_{k}^{-1 / q} \succeq \frac{\widehat{L}_{e}}{\left(\widehat{L}_{e} \bullet Y_{k}\right)^{1 / q}} \succeq \frac{\widehat{L}_{e}}{\operatorname{Dot}\left(\widehat{L}_{e}, Y_{k}\right)^{1 / q}}$. In addition, denoting by $\frac{\alpha \widehat{L}_{e}}{\operatorname{Dot}\left(\widehat{L}_{e}, Y_{k}\right)^{1 / q}}=P P^{T}$, we have $0 \preceq P^{T} Y_{k}^{1 / q} P \preceq \alpha I$.

Proof. We only prove the $X_{k}$ part because the $Y_{k}$ part is similar. We first compute

$$
\left\|X_{k}^{1 / 2 q} \widehat{L}_{e} X_{k}^{1 / 2 q}\right\|_{\text {spe }}^{q} \leq \operatorname{Tr}\left(\left(X_{k}^{1 / 2 q} \widehat{L}_{e} X_{k}^{1 / 2 q}\right)^{q}\right) \leq \operatorname{Tr}\left(X_{k}^{1 / 2}\left(\widehat{L}_{e}\right)^{q} X_{k}^{1 / 2}\right),
$$

where the last inequality follows from the Lieb-Thirring trace inequality.

Next, using the fact that $\widehat{L}_{e} \preceq I$, we obtain that $\left(\widehat{L}_{e}\right)^{q} \preceq \widehat{L}_{e}$. Therefore,

$$
\left\|X_{k}^{1 / 2 q} \widehat{L}_{e} X_{k}^{1 / 2 q}\right\|_{\text {spe }}^{q} \leq \operatorname{Tr}\left(X_{k}^{1 / 2} \widehat{L}_{e} X_{k}^{1 / 2}\right)=\widehat{L}_{e} \bullet X_{k}
$$

In other words, we have $X_{k}^{1 / 2 q} \widehat{L}_{e} X_{k}^{1 / 2 q} \preceq\left(\widehat{L}_{e} \bullet X_{k}\right)^{1 / q} \cdot I$ which means $X_{k}^{-1 / q} \succeq \frac{\widehat{L}_{e}}{\left(\widehat{L}_{e} \bullet X_{k}\right)^{1 / q}}$. We automatically have $\frac{\widehat{L}_{e}}{\left(\widehat{L}_{e} \bullet X_{k}\right)^{1 / q}} \succeq \frac{\widehat{L}_{e}}{\operatorname{Dot}\left(\widehat{L}_{e}, X_{k}\right)^{1 / q}}$ because $\operatorname{Dot}\left(\widehat{L}_{e}, X_{k}\right) \geq \widehat{L}_{e} \bullet X_{k}$.

To prove the second half, beginning from $X_{k}^{-1 / q} \succeq \frac{1}{\alpha} \cdot P P^{T}$, we left multiply it with $P^{T} X_{k}^{1 / q}$ and right multiply it with $X_{k}^{1 / q} P$, and obtain $P^{T} X_{k}^{1 / q} P \succeq \frac{1}{\alpha} \cdot P^{T} X_{k}^{1 / q} P P^{T} X_{k}^{1 / q} P$. Denoting by $D \stackrel{\text { def }}{=} P^{T} X_{k}^{1 / q} P$, we have $D \succeq \frac{1}{\alpha} D^{2}$, which immediately implies $0 \preceq D \preceq \alpha I$ as desired.

We have now finished the description of the algorithm. We remark here that $\operatorname{Tr} \widetilde{X}_{k+1}<\operatorname{Tr} X_{k}$ and $\operatorname{Tr} \widetilde{Y}_{k+1}>\operatorname{Tr} Y_{k}$. Therefore, since $\operatorname{Tr} X_{k+1}$ increases as $\delta_{X}$ increases, while $\operatorname{Tr} Y_{k+1}$ decreases as $\delta_{Y}$ increase, we conclude the existence of $\delta_{X}, \delta_{Y} \geq 0$ so that $\operatorname{Tr} X_{k+1}, \operatorname{Tr} Y_{k+1} \in\left[1,1+\varepsilon_{1}\right]$.

\section{F.3 Our Analysis}

We begin by reproving essentially the first half of Theorem 3.2: that is, to prove (E.3). We need to pay extra attention here since our $\operatorname{Tr} X_{k}$ and $\operatorname{Tr} Y_{k}$ do not precisely equal to 1 .

Lemma F.2. For every $U_{X} \succeq 0$ satisfying $\operatorname{Tr} U_{X} \leq 1$, and every $U_{Y} \succeq 0$ satisfying $\operatorname{Tr} U_{Y} \geq 1+\varepsilon_{1}$,

$$
\begin{aligned}
& \left\langle\frac{-\alpha \widehat{L}_{e_{k}}}{\operatorname{Dot}\left(\widehat{L}_{e_{k}}, X_{k}\right)^{1 / q}}, X_{k}-U_{X}\right\rangle \leq V_{\widetilde{X}_{k+1}}\left(X_{k}\right)+V_{\widetilde{X}_{k}}\left(U_{X}\right)-V_{\widetilde{X}_{k+1}}\left(U_{X}\right), \quad \text { and } \\
& \left\langle\frac{\alpha \widehat{L}_{e_{k}}}{\operatorname{Dot}\left(\widehat{L}_{e_{k}}, Y_{k}\right)^{1 / q}}, Y_{k}-U_{Y}\right\rangle \leq V_{\widetilde{Y}_{k+1}}\left(Y_{k}\right)+V_{\widetilde{Y}_{k}}\left(U_{Y}\right)-V_{\widetilde{Y}_{k+1}}\left(U_{Y}\right) .
\end{aligned}
$$

Proof. We first prove the $X_{k}$ part. By our choice of the regularizer, we have

$$
0=\nabla w\left(\widetilde{X}_{k+1}\right)-\nabla w\left(X_{k}\right)+\frac{-\alpha \widehat{L}_{e_{k}}}{\operatorname{Dot}\left(\widehat{L}_{e_{k}}, X_{k}\right)^{1 / q}}=-\widetilde{X}_{k+1}^{-1 / q}+X_{k}^{-1 / q}+\frac{-\alpha \widehat{L}_{e_{k}}}{\operatorname{Dot}\left(\widehat{L}_{e_{k}}, X_{k}\right)^{1 / q}} .
$$

Next, we obtain that

$$
\begin{aligned}
\left\langle\frac{-\alpha \widehat{L}_{e_{k}}}{\operatorname{Dot}\left(\widehat{L}_{e_{k}}, X_{k}\right)^{1 / q}}, X_{k}-U_{X}\right\rangle & =\left\langle\nabla w\left(X_{k}\right)-\nabla w\left(\widetilde{X}_{k+1}\right), X_{k}-U_{X}\right\rangle \\
& \stackrel{(1)}{=} V_{X_{k}}\left(U_{X}\right)-V_{\widetilde{X}_{k+1}}\left(U_{X}\right)+V_{\widetilde{X}_{k+1}}\left(X_{k}\right) \\
& \stackrel{2}{\leq} V_{\widetilde{X}_{k}}\left(U_{X}\right)-V_{\widetilde{X}_{k+1}}\left(U_{X}\right)+V_{\widetilde{X}_{k+1}}\left(X_{k}\right) .
\end{aligned}
$$


Above, (1) is due to the three-point equality of Bregman divergence, and (2) comes from

$$
\begin{aligned}
V_{X_{k}}\left(U_{X}\right)-V_{\widetilde{X}_{k}}\left(U_{X}\right) & \stackrel{(3)}{=}\left(X_{k}^{-1 / q}-\widetilde{X}_{k}^{-1 / q}\right) \bullet U_{X}+\frac{1}{q-1}\left(\operatorname{Tr} X_{k}^{1-1 / q}-\operatorname{Tr} \widetilde{X}_{k}^{1-1 / q}\right) \\
& \stackrel{(4)}{=} \delta_{X} \operatorname{Tr} U_{X}+\frac{1}{q-1} \sum_{i} \frac{1}{\lambda_{i}^{q-1}}-\frac{1}{\left(\lambda_{i}-\delta_{X}\right)^{q-1}} \\
& \stackrel{5}{\leq} \delta_{X} \operatorname{Tr} U_{X}-\delta_{X} \sum_{i} \frac{1}{\lambda_{i}^{q}} \stackrel{\stackrel{6}{\leq}}{\leq} 0
\end{aligned}
$$

Here, (3) is owing to the definition of Bregman divergence. (4) comes from the fact that $\widetilde{X}_{k+1}^{-1 / q}=$ $X_{k+1}^{-1 / q}-\delta_{X} I$, and the definition of choosing $\lambda_{i}$ to be the $i$-th eigenvalue of $X_{k+1}^{-1 / q}$. (5) follows from the convexity of $f(x)=x^{1-q}$ which implies $f\left(\lambda_{i}\right)-f\left(\lambda_{i}-\delta_{X}\right) \leq \nabla f\left(\lambda_{i}\right) \cdot \delta_{X}$. (6) is by our assumption of $\operatorname{Tr} U_{X} \leq 1$ as well as $\operatorname{Tr} X_{k+1}=\sum_{i} \frac{1}{\lambda_{i}^{q}} \geq 1$.

Similarly, for the $Y_{k}$ part, we can compute

$$
\begin{aligned}
\left\langle\frac{\alpha \widehat{L}_{e_{k}}}{\operatorname{Dot}\left(\widehat{L}_{e_{k}}, Y_{k}\right)^{1 / q}}, Y_{k}-U_{Y}\right\rangle & =\left\langle\nabla w\left(Y_{k}\right)-\nabla w\left(\widetilde{Y}_{k+1}\right), Y_{k}-U_{Y}\right\rangle \\
& \stackrel{(1)}{=} V_{Y_{k}}\left(U_{Y}\right)-V_{\widetilde{Y}_{k+1}}\left(U_{Y}\right)+V_{\widetilde{Y}_{k+1}}\left(Y_{k}\right) \\
& \stackrel{(2)}{\leq} V_{\widetilde{Y}_{k}}\left(U_{Y}\right)-V_{\widetilde{Y}_{k+1}}\left(U_{Y}\right)+V_{\widetilde{Y}_{k+1}}\left(Y_{k}\right) .
\end{aligned}
$$

Above, (1) is due to the three-point equality, and inequality (2) comes from

$$
\begin{aligned}
V_{Y_{k}}\left(U_{Y}\right)-V_{\widetilde{Y}_{k}}\left(U_{Y}\right) & \stackrel{(3)}{=}\left(Y_{k}^{-1 / q}-\widetilde{Y}_{k}^{-1 / q}\right) \bullet U_{Y}+\frac{1}{q-1}\left(\operatorname{Tr} Y_{k}^{1-1 / q}-\operatorname{Tr} \widetilde{Y}_{k}^{1-1 / q}\right) \\
& \stackrel{(4)}{=}-\delta_{Y} \operatorname{Tr} U_{Y}+\frac{1}{q-1} \sum_{i} \frac{1}{\lambda_{i}^{q-1}}-\frac{1}{\left(\lambda_{i}+\delta_{Y}\right)^{q-1}} \\
& \stackrel{(5)}{\leq}-\delta_{Y} \operatorname{Tr} U_{Y}+\delta_{Y} \sum_{i} \frac{1}{\lambda_{i}^{q}} \stackrel{\stackrel{(}{\leq}}{\leq} 0 .
\end{aligned}
$$

Here, (3) is owing to the definition of Bregman divergence. (4) comes from the fact that $\widetilde{Y}_{k+1}^{-1 / q}=$ $Y_{k+1}^{-1 / q}+\delta_{Y} I$, and the definition of choosing $\lambda_{i}$ to be the $i$-th eigenvalue of $Y_{k+1}^{-1 / q}$. (5) follows from the convexity of $f(x)=x^{1-q}$ which implies $f\left(\lambda_{i}\right)-f\left(\lambda_{i}+\delta_{Y}\right) \leq \nabla f\left(\lambda_{i}\right) \cdot\left(-\delta_{Y}\right)$. (6) is by our assumption of $\operatorname{Tr} U_{Y} \geq 1+\varepsilon_{1}$ as well as $\operatorname{Tr} Y_{k+1}=\sum_{i} \frac{1}{\lambda_{i}^{q}} \leq 1+\varepsilon_{1}$.

In a next step, we reprove essentially the second half of Theorem 3.2: that is, to provide upper bounds on $V_{\widetilde{X}_{k+1}}\left(X_{k}\right)$ and $V_{\widetilde{Y}_{k+1}}\left(Y_{k}\right)$ in Lemma F.3 and Lemma F.4.

Lemma F.3. As long as $q \geq 2$ and $\alpha \leq 1 / 2 q$, we have

$$
V_{\widetilde{X}_{k+1}}\left(X_{k}\right) \leq \frac{q}{2}\left(\alpha^{2}+O\left(q \alpha^{3}\right)\right) \cdot\left(\widehat{L}_{e_{k}} \bullet X_{k}\right)^{1-1 / q} .
$$

Proof. Suppose $\frac{\alpha \widehat{L}_{e_{k}}}{\operatorname{Dot}\left(\widehat{L}_{e_{k}}, X_{k}\right)^{1 / q}}=P P^{T}$. Then, using the Sherman-Morrison-Woodbury formula,

$$
\begin{aligned}
\operatorname{Tr} \widetilde{X}_{k+1}^{1-1 / q}=\operatorname{Tr}\left(\left(X_{k}^{-1 / q}-P P^{T}\right)^{-1}\right)^{q-1} & =\operatorname{Tr}\left(X_{k}^{1 / q}+X_{k}^{1 / q} P\left(I-P^{T} X_{k}^{1 / q} P\right)^{-1} P^{T} X_{k}^{1 / q}\right)^{q-1} \\
& \leq \operatorname{Tr}\left(X_{k}^{1 / q}+\frac{X_{k}^{1 / q} P P^{T} X_{k}^{1 / q}}{1-\alpha}\right)^{q-1}
\end{aligned}
$$


where the last inequality follows because $\left(I-P^{T} X_{k}^{1 / q} P\right)^{-1} \preceq \frac{1}{1-\alpha} I$ owing to Claim F.1. as well as $A \preceq B \Longrightarrow \operatorname{Tr} A^{n} \leq \operatorname{Tr} B^{n}$. We continue and write

$$
\begin{aligned}
\operatorname{Tr} \widetilde{X}_{k+1}^{1-1 / q} \leq \operatorname{Tr}\left(X_{k}^{1 / q}+\frac{X_{k}^{1 / q} P P^{T} X_{k}^{1 / q}}{1-\alpha}\right)^{q-1} & =\operatorname{Tr}\left(X_{k}^{1 / 2 q}\left(I+\frac{X_{k}^{1 / 2 q} P P^{T} X_{k}^{1 / 2 q}}{1-\alpha}\right) X_{k}^{1 / 2 q}\right)^{q-1} \\
& \leq \operatorname{Tr}\left(X_{k}^{(q-1) / 2 q}\left(I+\frac{X_{k}^{1 / 2 q} P P^{T} X_{k}^{1 / 2 q}}{1-\alpha}\right)^{q-1} X_{k}^{(q-1) / 2 q}\right) \\
& =\operatorname{Tr}\left(X_{k}^{1-1 / q}\left(I+\frac{X_{k}^{1 / 2 q} P P^{T} X_{k}^{1 / 2 q}}{1-\alpha}\right)^{q-1}\right),
\end{aligned}
$$

where the inequality uses the Lieb-Thirring trace inequality (which relies on the fact that $q-1 \geq 1$ ). Finally, denoting by $D \stackrel{\text { def }}{=} \frac{X_{k}^{1 / 2 q} P P^{T} X_{k}^{1 / 2 q}}{1-\alpha} \preceq \frac{\alpha}{1-\alpha} I$, we see that

$$
(I+D)^{q-1} \preceq I+(q-1) D+\left(\frac{(q-1)(q-2)}{2} \alpha+O\left(q^{3} \alpha^{2}\right)\right) D .
$$

This above matrix inequality can be proved by first turning into its eigenbasis, and then verifying that $(1+x)^{q-1} \leq 1+(q-1) x+\frac{(q-1)(q-2)}{2} \alpha x+O\left(q^{3} \alpha^{2}\right) x$ for all $x \in\left[0, \frac{\alpha}{1-\alpha}\right]$. (This uses the fact that $\alpha \leq 1 / 2 q)$. Next, using the above matrix inequality, we conclude that

$$
\begin{aligned}
\operatorname{Tr} \widetilde{X}_{k+1}^{1-1 / q} & \leq \operatorname{Tr}\left(X_{k}^{1-1 / q}\left(I+\frac{X_{k}^{1 / 2 q} P P^{T} X_{k}^{1 / 2 q}}{1-\alpha}\right)^{q-1}\right) \\
& \leq \operatorname{Tr}\left(X_{k}^{1-1 / q}\left(I+\left((q-1)+\frac{(q-1)(q-2)}{2} \alpha+O\left(q^{3} \alpha^{2}\right)\right) \frac{X_{k}^{1 / 2 q} P P^{T} X_{k}^{1 / 2 q}}{1-\alpha}\right)\right) \\
& =\operatorname{Tr} X_{k}^{1-1 / q}+(q-1) \frac{1+\frac{q-2}{2} \alpha+O\left(q^{2} \alpha^{2}\right)}{1-\alpha} X_{k} \bullet P P^{T} .
\end{aligned}
$$

Therefore,

$$
\begin{aligned}
V_{\widetilde{X}_{k+1}}\left(X_{k}\right) & =\widetilde{X}_{k+1}^{-1 / q} \bullet X_{k}+\frac{1}{q-1} \operatorname{Tr} \widetilde{X}_{k+1}^{1-1 / q}-\frac{q}{q-1} \operatorname{Tr} X_{k}^{1-1 / q} \\
& =\left(X_{k}^{-1 / q}-P P^{T}\right) \bullet X_{k}+\frac{1}{q-1} \operatorname{Tr} \widetilde{X}_{k+1}^{1-1 / q}-\frac{q}{q-1} \operatorname{Tr} X_{k}^{1-1 / q} \\
& =-P P^{T} \bullet X_{k}+\frac{1}{q-1}\left(\operatorname{Tr} \widetilde{X}_{k+1}^{1-1 / q}-\operatorname{Tr} X_{k}^{1-1 / q}\right) \\
& \leq P P^{T} \bullet X_{k}\left(-1+\frac{1+\frac{q-2}{2} \alpha+O\left(q^{2} \alpha^{2}\right)}{1-\alpha}\right) \\
& =\frac{q}{2}\left(\alpha+O\left(q \alpha^{2}\right)\right) \cdot P P^{T} \bullet X_{k} \\
& \leq \frac{q}{2}\left(\alpha^{2}+O\left(q \alpha^{3}\right)\right) \cdot\left(\widehat{L}_{e_{k}} \bullet X_{k}\right)^{1-1 / q} .
\end{aligned}
$$

Lemma F.4. As long as $q \geq 2$ and $\alpha \leq 1 / 2 q$, we have

$$
V_{\widetilde{Y}_{k+1}}\left(Y_{k}\right) \leq \frac{q}{2}\left(\alpha^{2}+O\left(\alpha^{3}\right)\right) \cdot\left(\widehat{L}_{e_{k}} \bullet Y_{k}\right)^{1-1 / q} .
$$

Proof. Suppose $\frac{\alpha \widehat{L}_{e_{k}}}{\operatorname{Dot}\left(\widehat{L}_{e_{k}}, Y_{k}\right)^{1 / q}}=P P^{T}$. Then, using the Sherman-Morrison-Woodbury formula,

$$
\begin{aligned}
\operatorname{Tr} \widetilde{Y}_{k+1}^{1-1 / q}=\operatorname{Tr}\left(\left(Y_{k}^{-1 / q}+P P^{T}\right)^{-1}\right)^{q-1} & =\operatorname{Tr}\left(Y_{k}^{1 / q}-Y_{k}^{1 / q} P\left(I+P^{T} Y_{k}^{1 / q} P\right)^{-1} P^{T} Y_{k}^{1 / q}\right)^{q-1} \\
& \leq \operatorname{Tr}\left(Y_{k}^{1 / q}-\frac{Y_{k}^{1 / q} P P^{T} Y_{k}^{1 / q}}{1+\alpha}\right)^{q-1}
\end{aligned}
$$


where the last inequality follows because $\left(I+P^{T} Y_{k}^{1 / q} P\right)^{-1} \succeq \frac{1}{1+\alpha} I$ owing to Claim F.1. as well as $A \preceq B \Longrightarrow \operatorname{Tr} A^{n} \leq \operatorname{Tr} B^{n}$. We continue and write

$$
\begin{aligned}
\operatorname{Tr} \widetilde{Y}_{k+1}^{1-1 / q} \leq \operatorname{Tr}\left(Y_{k}^{1 / q}-\frac{Y_{k}^{1 / q} P P^{T} Y_{k}^{1 / q}}{1+\alpha}\right)^{q-1} & =\operatorname{Tr}\left(Y_{k}^{1 / 2 q}\left(I-\frac{Y_{k}^{1 / 2 q} P P^{T} Y_{k}^{1 / 2 q}}{1+\alpha}\right) Y_{k}^{1 / 2 q}\right)^{q-1} \\
& \leq \operatorname{Tr}\left(Y_{k}^{(q-1) / 2 q}\left(I-\frac{Y_{k}^{1 / 2 q} P P^{T} Y_{k}^{1 / 2 q}}{1+\alpha}\right)^{q-1} Y_{k}^{(q-1) / 2 q}\right) \\
& =\operatorname{Tr}\left(Y_{k}^{1-1 / q}\left(I-\frac{Y_{k}^{1 / 2 q} P P^{T} Y_{k}^{1 / 2 q}}{1+\alpha}\right)^{q-1}\right),
\end{aligned}
$$

where the inequality again uses the Lieb-Thirring trace inequality (which relies on the fact that $q-1 \geq 1)$. Denoting by $D \stackrel{\text { def }}{=} \frac{Y_{k}^{1 / 2 q} P P^{T} Y_{k}^{1 / 2 q}}{1+\alpha} \preceq \frac{\alpha}{1+\alpha} I$, we see that

$$
(I-D)^{q-1} \preceq I-(q-1) D+\frac{(q-1)(q-2) \alpha}{2(1+\alpha)} D .
$$

This above matrix inequality can be proved by first turning into its eigenbasis, and then verifying that $(1-x)^{q-1} \leq 1-(q-1) x+\frac{(q-1)(q-2)}{2} \frac{\alpha}{1+\alpha} x$ for all $x \in\left[0, \frac{\alpha}{1+\alpha}\right]$. (This uses the fact that $\alpha \leq 1 / 2 q)$. Next, using the above matrix inequality, we conclude that

$$
\begin{aligned}
\operatorname{Tr} \widetilde{Y}_{k+1}^{1-1 / q} & \leq \operatorname{Tr}\left(Y_{k}^{1-1 / q}\left(I-\frac{Y_{k}^{1 / 2 q} P P^{T} Y_{k}^{1 / 2 q}}{1+\alpha}\right)^{q-1}\right) \\
& \leq \operatorname{Tr}\left(Y_{k}^{1-1 / q}\left(I-(q-1)\left(1-\frac{(q-2) \alpha}{2(1+\alpha)}\right) \frac{Y_{k}^{1 / 2 q} P P^{T} Y_{k}^{1 / 2 q}}{1+\alpha}\right)\right) \\
& =\operatorname{Tr} Y_{k}^{1-1 / q}-(q-1)\left(1-\frac{(q-2) \alpha}{2(1+\alpha)}\right) \frac{1}{1+\alpha} Y_{k} \bullet P P^{T} .
\end{aligned}
$$

Therefore,

$$
\begin{aligned}
V_{\widetilde{Y}_{k+1}}\left(Y_{k}\right) & =\widetilde{Y}_{k+1}^{-1 / q} \bullet Y_{k}+\frac{1}{q-1} \operatorname{Tr} \widetilde{Y}_{k+1}^{1-1 / q}-\frac{q}{q-1} \operatorname{Tr} Y_{k}^{1-1 / q} \\
& =\left(Y_{k}^{-1 / q}+P P^{T}\right) \bullet Y_{k}+\frac{1}{q-1} \operatorname{Tr} \widetilde{Y}_{k+1}^{1-1 / q}-\frac{q}{q-1} \operatorname{Tr} Y_{k}^{1-1 / q} \\
& =P P^{T} \bullet Y_{k}+\frac{1}{q-1}\left(\operatorname{Tr} \widetilde{Y}_{k+1}^{1-1 / q}-\operatorname{Tr} Y_{k}^{1-1 / q}\right) \\
& \leq P P^{T} \bullet Y_{k}\left(1-\frac{\left(1-\frac{(q-2) \alpha}{2(1+\alpha)}\right)}{1+\alpha}\right) \\
& =\frac{q}{2}\left(\alpha+O\left(\alpha^{2}\right)\right) \cdot P P^{T} \bullet Y_{k} \\
& \leq \frac{q}{2}\left(\alpha^{2}+O\left(\alpha^{3}\right)\right) \cdot\left(\widehat{L}_{e_{k}} \bullet Y_{k}\right)^{1-1 / q} .
\end{aligned}
$$

Theorem F.5. Suppose $\varepsilon<\frac{1}{4 \sqrt{q}}$ and $\varepsilon_{1}<\frac{1}{2}$, and we choose $\alpha=\frac{\varepsilon \sqrt{2}}{\sqrt{q-1}}$ and $T=\frac{n}{\varepsilon^{2}}$. Then, the matrix $M_{Y} \stackrel{\text { def }}{=} \sum_{k=0}^{T-1} \frac{\widehat{L}_{e_{k}}}{\operatorname{Dot}\left(\widehat{L}_{e_{k}}, Y_{k}\right)^{1 / q}}$ satisfies that

$$
\lambda_{\max }\left(M_{Y}\right)-\lambda_{\min }\left(M_{Y}\right) \leq \lambda_{\min }\left(M_{Y}\right) \cdot\left(\sqrt{\frac{8 q^{2}}{q-1}} \cdot \varepsilon+O\left(\varepsilon_{1}+q \varepsilon^{2}\right)\right) .
$$

This theorem provides the sparsification guarantee to our Theorem 1 and 2 . We shall provide its running time guarantee in the next section. 
Proof. Define matrices $M_{X} \stackrel{\text { def }}{=} \sum_{k=0}^{T-1} \frac{\widehat{L}_{e_{k}}}{\operatorname{Dot}\left(\widehat{L}_{e_{k}}, X_{k}\right)^{1 / q}}$ and $M_{Y} \stackrel{\text { def }}{=} \sum_{k=0}^{T-1} \frac{\widehat{L}_{e_{k}}}{\operatorname{Dot}\left(\widehat{L}_{e_{k}}, Y_{k}\right)^{1 / q}}$. Also, denote by $\xi \stackrel{\text { def }}{=} \frac{q}{2}\left(\alpha+O\left(q \alpha^{2}\right)\right)$.

We are now ready to rededuce (5.3) and (5.4) in Section 5.

Combining Lemma F.2 and Lemma F.3, and telescoping for $k=0,1, \ldots, T-1$, we have

$$
\begin{aligned}
\forall U_{X} \succeq 0 \text { satisfying } \operatorname{Tr} U_{X}=1, \quad M_{X} \bullet U_{X} & \leq \frac{V_{\widetilde{X}_{0}}\left(U_{X}\right)}{\alpha}+(1+\xi) \sum_{k=0}^{T-1}\left(\widehat{L}_{e_{k}} \bullet X_{k}\right)^{1-1 / q} \\
& \leq \frac{q n^{1 / q}}{(q-1) \alpha}+(1+\xi) \sum_{k=0}^{T-1}\left(\widehat{L}_{e_{k}} \bullet X_{k}\right)^{1-1 / q} .
\end{aligned}
$$

Above, the second inequality uses the fact that $V_{\widetilde{X}_{0}}\left(U_{X}\right) \leq \frac{q}{q-1} n^{1 / q}$.

Combining Lemma F.2 and Lemma F.4, and telescoping for $k=0,1, \ldots, T-1$, we have

$$
\begin{aligned}
\forall U_{Y} \succeq 0, \operatorname{Tr} U_{Y}=1+\varepsilon_{1}, \quad M_{Y} \bullet U_{Y} & \geq-\frac{V_{\widetilde{Y}_{0}}\left(U_{Y}\right)}{\alpha}+(1-\xi) \sum_{k=0}^{T-1}\left(\widehat{L}_{e_{k}} \bullet Y_{k}\right)^{1-1 / q} \\
& \geq-\frac{q\left(1+\varepsilon_{1}\right) n^{1 / q}}{(q-1) \alpha}+(1-\xi) \sum_{k=0}^{T-1}\left(\widehat{L}_{e_{k}} \bullet Y_{k}\right)^{1-1 / q} .
\end{aligned}
$$

Above, the second inequality uses the fact that $V_{\widetilde{Y}_{0}}\left(U_{Y}\right) \leq \frac{q\left(1+\varepsilon_{1}\right)}{q-1} n^{1 / q}$.

Similar to the proof in Section 5. we provide deduce our eigenvalue inequality in two steps.

Lowerbounding $\boldsymbol{\lambda}_{\min }\left(M_{\boldsymbol{Y}}\right)$. Since we have assumed each $\operatorname{Tr} \widehat{L}_{e}$ to be at least $1-\varepsilon_{1}$, we have

$$
\operatorname{Tr}\left(M_{X}\right)=\sum_{k=0}^{T-1} \frac{\operatorname{Tr} \widehat{L}_{e_{k}}}{\operatorname{Dot}\left(\widehat{L}_{e_{k}}, X_{k}\right)^{1 / q}} \geq \frac{1-\varepsilon_{1}}{\left(1+\varepsilon_{1}\right)^{1 / q}} \sum_{k=0}^{T-1} \frac{1}{\left(\widehat{L}_{e_{k}} \bullet X_{k}\right)^{1 / q}} .
$$

Denoting by $a_{k}=\widehat{L}_{e_{k}} \bullet X_{k}$, we can write $\operatorname{Tr}\left(M_{X}\right) \geq \frac{1-\varepsilon_{1}}{\left(1+\varepsilon_{1}\right)^{1 / q}} \sum_{k=0}^{T-1} \frac{1}{a_{k}^{1 / q}}$. Applying (F.2) with the choice of $U_{X}=\frac{1}{n} I=X_{0}$, we have

$$
\frac{1-\varepsilon_{1}}{n\left(1+\varepsilon_{1}\right)^{1 / q}} \sum_{k=0}^{T-1} \frac{1}{a_{k}^{1 / q}} \leq \frac{1}{n} \operatorname{Tr} M_{X}=M_{X} \bullet U_{X} \leq(1+\xi) \sum_{k=0}^{T-1}\left(\widehat{L}_{e_{k}} \bullet X_{k}\right)^{1-1 / q} \leq(1+\xi) \sum_{k=0}^{T-1} a_{k}^{1-1 / q} .
$$

Using the above inequality we obtain

$$
\begin{aligned}
\sum_{k=0}^{T-1} a_{k}^{1-1 / q} & \geq \frac{1}{\left(n(1+\xi)\left(1+\varepsilon_{1}\right)^{1 / q}\left(1-\varepsilon_{1}\right)^{-1}\right)^{1-1 / q}}\left(\sum_{k=0}^{T-1} a_{k}^{1-1 / q}\right)^{1 / q}\left(\sum_{k=0}^{T-1} \frac{1}{a_{k}^{1 / q}}\right)^{1-1 / q} \\
& \geq \frac{T}{n^{1-1 / q}(1+\xi)^{1-1 / q}\left(1+\varepsilon_{1}\right)^{1 / q-1 / q^{2}}\left(1-\varepsilon_{1}\right)^{1 / q-1}},
\end{aligned}
$$

where the last inequality follows from Hölder's inequality. If we choose $T=\frac{n}{\varepsilon^{2}}$, this immediately gives

$$
\sum_{k=0}^{T-1}\left(\widehat{L}_{e_{k}} \bullet X_{k}\right)^{1-1 / q}=\sum_{k=0}^{T-1} a_{k}^{1-1 / q} \geq \frac{n^{1 / q}}{\varepsilon^{2}}\left(1-O\left(q \alpha+\varepsilon_{1}\right)\right)
$$

Finally, substituting (F.5) into (F.4), and choosing $U_{Y}$ so that $M_{Y} \bullet U_{Y}=\left(1+\varepsilon_{1}\right) \lambda_{\min }\left(M_{Y}\right)$, 
we have

$$
\begin{aligned}
\left(1+\varepsilon_{1}\right) \lambda_{\min }\left(M_{Y}\right) & \geq-\frac{q\left(1+\varepsilon_{1}\right) n^{1 / q}}{(q-1) \alpha}+(1-\xi) \frac{1}{\left(1+\varepsilon_{1}\right)^{3-3 / q}} \sum_{k=0}^{T-1}\left(\widehat{L}_{e_{k}} \bullet X_{k}\right)^{1-1 / q} \\
& \geq-\frac{2 q n^{1 / q}}{(q-1) \alpha}+(1-\xi) \frac{1}{\left(1+\varepsilon_{1}\right)^{3-3 / q}} \frac{n^{1 / q}}{\varepsilon^{2}}\left(1-O\left(q \alpha+\varepsilon_{1}\right)\right) \\
& \geq-\frac{2 q n^{1 / q}}{(q-1) \alpha}+\frac{n^{1 / q}}{\varepsilon^{2}}\left(1-O\left(q \alpha+\varepsilon_{1}\right)\right) \\
& \geq \frac{n^{1 / q}}{\varepsilon^{2}}\left(1-O\left(q \alpha+\varepsilon_{1}+\varepsilon^{2} / \alpha\right)\right) .
\end{aligned}
$$

Above, the first inequality is due to our choice of $e_{k}$ which satisfies

$$
\left(1+\varepsilon_{1}\right)^{3} \widehat{L}_{e_{k}} \bullet Y_{k} \geq\left(1+\varepsilon_{1}\right)^{2} \operatorname{Dot}\left(\widehat{L}_{e_{k}}, Y_{k}\right) \geq \operatorname{Dot}\left(\widehat{L}_{e_{k}}, X_{k}\right) \geq \widehat{L}_{e_{k}} \bullet X_{k}
$$

Upper bounding $\lambda_{\max }\left(M_{Y}\right)-\lambda_{\min }\left(M_{Y}\right)$. This time, combining (F.3) and (F.4), as well as using (F.7), we compute that

$\frac{1}{1+\xi}\left(M_{Y} \bullet U_{X}-\frac{q n^{1 / q}}{(q-1) \alpha}\right) \leq \frac{1}{1+\xi}\left(M_{X} \bullet U_{X}-\frac{q n^{1 / q}}{(q-1) \alpha}\right) \leq \frac{\left(1+\varepsilon_{1}\right)^{3-3 / q}}{1-\xi}\left(M_{Y} \bullet U_{Y}+\frac{q\left(1+\varepsilon_{1}\right) n^{1 / q}}{(q-1) \alpha}\right)$.

Choosing $U_{X}$ so that $M_{Y} \bullet U_{X}=\lambda_{\max }\left(M_{Y}\right)$, and $U_{Y}$ so that $M_{Y} \bullet U_{Y}=\left(1+\varepsilon_{1}\right) \lambda_{\min }\left(M_{Y}\right)$, we can rewrite the above inequality as

$$
\frac{1}{1+\xi}\left(\lambda_{\max }\left(M_{Y}\right)-\frac{q n^{1 / q}}{(q-1) \alpha}\right) \leq \frac{\left(1+\varepsilon_{1}\right)^{3-3 / q}}{1-\xi}\left(1+\varepsilon_{1}\right)\left(\lambda_{\min }\left(M_{Y}\right)+\frac{q n^{1 / q}}{(q-1) \alpha}\right) .
$$

To turn this joint multiplicative-additive error into a purely multiplicative one, we further rewrite it as

$$
\begin{aligned}
\lambda_{\max }\left(M_{Y}\right)-\lambda_{\min }\left(M_{Y}\right) & \leq \frac{2 \xi+O\left(\varepsilon_{1}\right)}{1-\xi} \lambda_{\min }\left(M_{Y}\right)+\frac{1+\xi+O\left(\varepsilon_{1}\right)}{1-\xi} \frac{q n^{1 / q}}{(q-1) \alpha}+\frac{q n^{1 / q}}{(q-1) \alpha} \\
& =\frac{2 \xi+O\left(\varepsilon_{1}\right)}{1-\xi} \lambda_{\min }\left(M_{Y}\right)+\frac{2 q}{q-1} \frac{1+O\left(\varepsilon_{1}\right)}{1-\xi} \frac{n^{1 / q}}{\alpha} \\
& \leq \frac{2 \xi+O\left(\varepsilon_{1}\right)}{1-\xi} \lambda_{\min }\left(M_{Y}\right)+\frac{2 q}{q-1} \cdot \lambda_{\min }\left(M_{Y}\right) \frac{\varepsilon^{2}}{\alpha}\left(1+O\left(q \alpha+\varepsilon_{1}+\varepsilon^{2} / \alpha\right)\right) \\
& =\lambda_{\min }\left(M_{Y}\right) \cdot\left(q \alpha+\frac{2 q}{q-1} \frac{\varepsilon^{2}}{\alpha}+O\left(\varepsilon_{1}+q \varepsilon^{2}+\varepsilon_{1} \varepsilon^{2} / \alpha+\varepsilon^{4} / \alpha^{2}+q^{2} \alpha^{2}\right)\right) .
\end{aligned}
$$

Above, the second inequality uses (F.6). Now, it is clear that by choosing $\alpha=\frac{\varepsilon \sqrt{2}}{\sqrt{q-1}} \leq \frac{1}{2 q}$, we have

$$
\begin{aligned}
\lambda_{\max }\left(M_{Y}\right)-\lambda_{\min }\left(M_{Y}\right) & \leq \lambda_{\min }\left(M_{Y}\right) \cdot\left(\sqrt{\frac{8 q^{2}}{q-1}} \cdot \varepsilon+O\left(\varepsilon_{1}+q \varepsilon^{2}+\varepsilon_{1} \varepsilon \sqrt{q}\right)\right) \\
& \leq \lambda_{\min }\left(M_{Y}\right) \cdot\left(\sqrt{\frac{8 q^{2}}{q-1}} \cdot \varepsilon+O\left(\varepsilon_{1}+q \varepsilon^{2}\right)\right) .
\end{aligned}
$$

\section{F.4 An Additional Property}

Recall that in the previous subsection, we have constructed $M_{X}$ and $M_{Y}$ and proved that $\lambda_{\min }\left(M_{Y}\right)$ (and in fact $\lambda_{\min }\left(M_{Y}\right)$ as well) is at least $\Omega\left(n^{1 / q} / \varepsilon^{2}\right)$. In this subsection, we shall show that $\lambda_{\max }\left(M_{X}\right)$ and $\lambda_{\max }\left(M_{Y}\right)$ can be made at most $O\left(n^{1 / q} / \varepsilon^{2}\right)$ as well. While this additional property is not needed for proving Theorem F.5. it shall become useful for proving the desired running time in the next section (see Appendix G). 
The following lemma ensures that if we stop the algorithm "whenever we are done", and thus choose possibly less than $n / \varepsilon^{2}$ matrices, then, $\lambda_{\max }\left(M_{X}\right)$ and $\lambda_{\max }\left(M_{Y}\right)$ can be properly upper bounded.

Lemma F.6. If one stops the algorithm either when $T=\frac{n}{\varepsilon^{2}}$ iterations are performed, or when the first time that $\sum_{k=0}^{T-1} \operatorname{Dot}\left(\widehat{L}_{e_{k}}, X_{k}\right)^{1-1 / q} \geq \frac{n^{1 / q}}{\varepsilon^{2}}$ is satisfied, then the same result of Theorem F.5 can be obtained, while we have an extra guarantee

$$
\lambda_{\max }\left(M_{X}\right), \lambda_{\max }\left(M_{Y}\right) \leq O\left(\frac{n^{1 / q}}{\varepsilon^{2}}\right) .
$$

Proof. Recall that in the proof of Theorem F.5. we have only used the choice of $T=\frac{n}{\varepsilon^{2}}$ to deduce (F.5). For this reason, if instead of choosing exactly $T=\frac{n}{\varepsilon^{2}}$ matrices, we

$$
\text { stop the algorithm at the first time } T \text { such that } \sum_{k=0}^{T-1} \operatorname{Dot}\left(\widehat{L}_{e_{k}}, X_{k}\right)^{1-1 / q} \geq \frac{n^{1 / q}}{\varepsilon^{2}} \text { is satisfied, }
$$

then we automatically have

$$
\sum_{k=0}^{T-1}\left(\widehat{L}_{e_{k}} \bullet X_{k}\right)^{1-1 / q} \geq \frac{n^{1 / q}}{\varepsilon^{2}}\left(1-O\left(\varepsilon_{1}\right)\right)
$$

Replacing (F.5) with the above lower bound, all results claimed in Theorem F.5 remain true.

In the rest of the proof, we will show that this early termination rule ensures a good upper bound on $\lambda_{\max }\left(M_{X}\right)$ and $\lambda_{\max }\left(M_{Y}\right)$. Indeed, at the time the algorithm is terminated, we must have

$$
\sum_{k=0}^{T-1}\left(\widehat{L}_{e_{k}} \bullet X_{k}\right)^{1-1 / q} \leq \sum_{k=0}^{T-1} \operatorname{Dot}\left(\widehat{L}_{e_{k}}, X_{k}\right)^{1-1 / q} \leq \frac{n^{1 / q}}{\varepsilon^{2}}+O(1)
$$

This is because, since $\widehat{L}_{e_{k}} \bullet X_{k} \leq I \bullet X_{k}=1$ and thus $\operatorname{Dot}\left(\widehat{L}_{e_{k}}, X_{k}\right)^{1-1 / q} \leq O(1)$, the value $\sum_{k=0}^{T-1} \operatorname{Dot}\left(\widehat{L}_{e_{k}}, X_{k}\right)^{1-1 / q}$ is incremented by at most $O(1)$ at each iteration. As a consequence, at the first iteration it exceeds $n^{1 / q} / \varepsilon^{2}$, the summation must be at least $n^{1 / q} / \varepsilon^{2}+O(1)$.

Next, substituting (F.9) into (F.3), and choosing $U_{X}$ so that $M_{X} \bullet U_{X}=\lambda_{\max }\left(M_{X}\right)$, we have

$$
\lambda_{\max }\left(M_{X}\right) \leq \frac{q n^{1 / q}}{(q-1) \alpha}+(1+\xi) \frac{n^{1 / q}}{\varepsilon^{2}}+O(1)=O\left(\frac{n^{1 / q}}{\varepsilon^{2}}\right) .
$$

Finally, recalling that we have chosen $\operatorname{Dot}\left(\widehat{L}_{e_{k}}, X_{k}\right) \leq\left(1+\varepsilon_{1}\right)^{2} \operatorname{Dot}\left(\widehat{L}_{e_{k}}, Y_{k}\right)$, this ensures that $\left(1+\varepsilon_{1}\right)^{2} M_{X} \succeq M_{Y}$. In sum, we obtain that $\lambda_{\max }\left(M_{Y}\right) \leq O\left(\lambda_{\max }\left(M_{X}\right)\right) \leq O\left(\frac{n^{1 / q}}{\varepsilon^{2}}\right)$.

\section{G Efficient Implementation for Graph Sparsifications}

Recall from Appendix F that in order to implement the algorithm described in Theorem F.5. we need to

(C1) Ensure that each $\operatorname{Tr} \widehat{L}_{e}$ is in $\left[1-\varepsilon_{1}, 1\right]$.

(C2) Compute at each iteration two reals $c^{X}, c^{Y} \in \mathbb{R}$ satisfying that $\operatorname{Tr} X_{k} \in\left[1,1+\varepsilon_{1}\right]$ and $\operatorname{Tr} Y_{k} \in$ $\left[1,1+\varepsilon_{1}\right]$, where

$$
X_{k} \stackrel{\text { def }}{=}\left(c^{X} \cdot I-\sum_{j=0}^{k-1} \frac{\alpha \widehat{L}_{e_{j}}}{\operatorname{Dot}\left(\widehat{L}_{e_{j}}, X_{j}\right)^{1 / q}}\right)^{-q} \text { and } \quad Y_{k} \stackrel{\text { def }}{=}\left(\sum_{j=0}^{k-1} \frac{\alpha \widehat{L}_{e_{j}}}{\operatorname{Dot}\left(\widehat{L}_{e_{j}}, Y_{j}\right)^{1 / q}}-c^{Y} \cdot I\right)^{-q} .
$$


(C3) Compute at each iteration $\operatorname{Dot}\left(\widehat{L}_{e}, X_{k}\right)$ and $\operatorname{Dot}\left(\widehat{L}_{e}, Y_{k}\right)$ which satisfy

$$
\widehat{L}_{e} \bullet X_{k} \leq \operatorname{Dot}\left(\widehat{L}_{e}, X_{k}\right) \leq\left(1+\varepsilon_{1}\right) \widehat{L}_{e} \bullet X_{k} \quad \text { and } \quad \widehat{L}_{e} \bullet Y_{k} \leq \operatorname{Dot}\left(\widehat{L}_{e}, Y_{k}\right) \leq\left(1+\varepsilon_{1}\right) \widehat{L}_{e} \bullet Y_{k}
$$

In this section, we suppose that we are dealing with a spectral graph sparsification instance (see Appendix B). In other words, we use $I$ to denote $I_{\operatorname{im}\left(L_{G}\right)}$, and have $\widehat{L}_{e}=\frac{L_{G}^{-1 / 2} L_{e} L_{G}^{-1 / 2}}{w_{e}}$, where $w_{e}=L_{G}^{-1} \bullet L_{e}$ is the effective resistance of edge $e \in[m]$.

Knowing this scaling factor $w_{e}$ is somewhat important, because we need to ensure that $\operatorname{Tr} \widehat{L}_{e}$ is between $1-\varepsilon_{1}$ and 1 according to (C1). Fortunately, Spielman and Srivastava SS11] have given an algorithm that runs in nearly-linear time, and produces the effective resistances $L_{G}^{-1} \bullet L_{e}$ up to a multiplicative error of $1+\varepsilon_{1}$ for all edges $e \in[m]$, with probability at least $1-n^{-\Omega(1)}$.

In other words, we can denote by $\widehat{L}_{e}=\frac{L_{G}^{-1 / 2} L_{e} L_{G}^{-1 / 2}}{w_{e}}$, where each $w_{e}$ only needs to be between $\left(1-\varepsilon_{1}\right) \cdot L_{G}^{-1} \bullet L_{e}$ and $L_{G}^{-1} \bullet L_{e}$.

We next wish to show how to implement (C2) and (C3) efficiently. Before that, let us claim that

Lemma G.1. Regardingless of how (C2) and (C3) are implemented, for all iterations, $c^{X}, c^{Y} \leq$ $O\left(\alpha \frac{n^{1 / q}}{\varepsilon^{2}}\right)=O\left(\frac{n^{1 / q}}{\sqrt{q} \varepsilon}\right)$.

Proof. It is first easy to see that $c^{Y} \leq \alpha \cdot \lambda_{\max }\left(M_{Y}\right) \leq O\left(\alpha \frac{n^{1 / q}}{\varepsilon^{2}}\right)$ owing to Lemma F.6. Next, since $\operatorname{Tr} X_{k} \geq 1$, we must have

$$
c^{X} \leq \lambda_{\max }\left(\sum_{j=0}^{k-1} \frac{\alpha \widehat{L}_{e_{j}}}{\operatorname{Dot}\left(\widehat{L}_{e_{j}}, X_{j}\right)^{1 / q}}\right)+n^{1 / q} \leq \alpha \cdot \lambda_{\max }\left(M_{X}\right)+n^{1 / q} \leq O\left(\alpha \frac{n^{1 / q}}{\varepsilon^{2}}\right) .
$$

Now, we are ready to prove the main theorem of this section.

Theorem G.2. In an amortized $d^{a}$ running time of $\widetilde{O}\left(\sqrt{q} n^{1 / q} m / \varepsilon_{1}^{2} \varepsilon\right)$ per iteration, we can implement (C2) and (C3) with probability at least $1-n^{-\Omega(1)}$.

Combining this with the fact that there are at mots $\frac{n}{\varepsilon^{2}}$ iterations, the total running time of our graph sparsification algorithm is

$$
\widetilde{O}\left(\frac{\sqrt{q} n^{1+1 / q} m}{\varepsilon_{1}^{2} \varepsilon^{3}}\right)
$$

${ }^{a}$ This amortization can be removed, but will result in a slightly more involved implementation to analyze.

Our proof below will make frequent uses of Lemma G.3 and Lemma G.4. two independent lemmas regarding how to efficiently compute matrix inversions of the form $(c I-A)^{-q}$ as well as $(A-c I)^{-q}$. The statements and proofs of these two lemmas are deferred to Appendix G.1.

Proof. Both (C2) and (C3) are trivially implementable when $k=0$, because $X_{0}=Y_{0}=\frac{1}{n} I$.

Suppose that both of them are implementable at iteration $k-1$. We proceed in 4 steps to prove that they are implementable at iteration $k$ as well.

- Step I: prove (C3) for computing $\operatorname{Dot}\left(\widehat{L}_{e}, X_{k}\right)$.

Suppose $X_{k}$ is given in the form of $X_{k} \stackrel{\text { def }}{=}\left(c^{X} \cdot I-\sum_{j=0}^{k-1} \frac{\alpha \widehat{L}_{e_{j}}}{\operatorname{Dot}\left(\widehat{L}_{e_{j}}, X_{j}\right)^{1 / q}}\right)^{-q}$ for some $c^{X}>0$, and it satisfies $\operatorname{Tr} X_{k} \in\left[1,1+\varepsilon_{1}\right]$. (This is done by the inductive assumption.) 
Since $\operatorname{Tr} X_{k} \leq 1+\varepsilon_{1} \leq 3 / 2$, we must have

$$
X_{k}^{-1 / q}=c^{X} \cdot I-\sum_{j=0}^{k-1} \frac{\alpha \widehat{L}_{e_{j}}}{\operatorname{Dot}\left(\widehat{L}_{e_{j}}, X_{j}\right)^{1 / q}} \succeq \frac{2}{3} I .
$$

This inequality ensures that we can compute $X_{k} \bullet \widehat{L}_{e}$ approximately (up to $1+\varepsilon_{1}$ error) using Lemma G.3. Since $c^{X}$ is no more than $O\left(n^{1 / q} / \sqrt{q} \varepsilon\right)$ owing to Lemma G.1. the running time for computing $X_{k} \bullet \widehat{L}_{e}$ for all edges $e \in E$ is $\widetilde{O}\left(c^{X} q m / \varepsilon_{1}^{2}\right)=\widetilde{O}\left(\sqrt{q} n^{1 / q} m / \varepsilon_{1}^{2} \varepsilon\right)$.

- Step II: prove (C3) for computing $\operatorname{Dot}\left(\widehat{L}_{e}, Y_{k}\right)$.

Suppose $Y_{k}$ is given in the form of $Y_{k} \stackrel{\text { def }}{=}\left(\sum_{j=0}^{k-1} \frac{\alpha \widehat{L}_{e_{j}}}{\operatorname{Dot}\left(\widehat{L}_{e_{j}}, Y_{j}\right)^{1 / q}}-c^{Y} \cdot I\right)^{-q}$ for some real $c^{Y}$, and it satisfies $\operatorname{Tr} Y_{k} \in\left[1,1+\varepsilon_{1}\right]$. (This is done by the inductive assumption.) Since $\operatorname{Tr} Y_{k} \leq 1+\varepsilon_{1} \leq 3 / 2$, we must have

$$
Y_{k}^{-1 / q}=\sum_{j=0}^{k-1} \frac{\alpha \widehat{L}_{e_{j}}}{\operatorname{Dot}\left(\widehat{L}_{e_{j}}, Y_{j}\right)^{1 / q}}-c^{Y} \cdot I \succeq \frac{2}{3} I .
$$

This inequality ensures that we can compute $Y_{k} \bullet \widehat{L}_{e}$ approximately (up to $1+\varepsilon_{1}$ error) using Lemma G.4. Since $c^{Y}$ is no more than $O\left(n^{1 / q} / \sqrt{q} \varepsilon\right)$ owing to Lemma G.1, the running time for computing $Y_{k} \bullet \widehat{L}_{e}$ for all edges $e \in E$ is $\widetilde{O}\left(c^{Y} q m / \varepsilon_{1}^{2}\right)=\widetilde{O}\left(\sqrt{q} n^{1 / q} m / \varepsilon_{1}^{2} \varepsilon\right)$.

- Step III: prove $(\mathrm{C} 2)$ for $X_{k}$.

Suppose that $X_{k-1} \stackrel{\text { def }}{=}\left(b^{X} \cdot I-\sum_{j=0}^{k-2} \frac{\alpha \widehat{L}_{e_{j}}}{\operatorname{Dot}\left(\widehat{L}_{e_{j}}, X_{j}\right)^{1 / q}}\right)^{-q}$. Since $\operatorname{Tr} X_{k-1} \leq 1+\varepsilon_{1} \leq 3 / 2$, we must have

$$
X_{k-1}^{-1 / q}=b^{X} \cdot I-\sum_{j=0}^{k-2} \frac{\alpha \widehat{L}_{e_{j}}}{\operatorname{Dot}\left(\widehat{L}_{e_{j}}, X_{j}\right)^{1 / q}} \succeq \frac{2}{3} I .
$$

Recall that we have proved that $X_{k-1}^{-1 / q} \succeq \frac{\widehat{L}_{e_{j}}}{\operatorname{Dot}\left(\widehat{L}_{e_{j}}, X_{j}\right)^{1 / q}}$ (see Claim F.1), combining it with the inequality above and the fact that $\alpha<1 / 4$, we have

$$
b^{X} \cdot I-\sum_{j=0}^{k-1} \frac{\alpha \widehat{L}_{e_{j}}}{\operatorname{Dot}\left(\widehat{L}_{e_{j}}, X_{j}\right)^{1 / q}} \succeq \frac{1}{2} I .
$$

Now, we are ready to perform a binary search to find $c^{X}$. If one selects $c^{X}=b^{X}$, he will get $\operatorname{Tr} X_{k} \geq \operatorname{Tr} X_{k-1} \geq 1$, and therefore $c^{X}=b^{X}$ is a good lower bound for the choice of $c^{X}$. On the other hand, if one selects $c^{X}=b^{X}+n^{1 / q}$, he will get $\operatorname{Tr} X_{k} \leq \operatorname{Tr}\left(n^{1 / q} I\right)^{-q}=1$, so $b^{X}+n^{1 / q}$ is a good upper bound for the choice of $c^{X}$.

In sum, we can binary search $c^{X}$ in the interval of $\left[b^{X}, b^{X}+n^{1 / q}\right]$. For each such value of $c^{X}$ in the process of the binary search, since $c^{X}$ is no more than $O\left(n^{1 / q} / \sqrt{q} \varepsilon\right)$ as per Lemma G.1. one can apply Lemma G.3 and approximately compute $\operatorname{Tr}\left(X_{k}\right)=\sum_{e} X_{k} \bullet \widehat{L}_{e}$ up to a multiplicative error of $1+\varepsilon_{1}$, in time $\widetilde{O}\left(c^{X} q m / \varepsilon_{1}^{2}\right)=\widetilde{O}\left(\sqrt{q} n^{1 / q} m / \varepsilon_{1}^{2} \varepsilon\right)$.

Since the overhead for the binary search is $\widetilde{O}(1)$, the total running time to compute $c^{X}$ at an iteration is $\widetilde{O}\left(\sqrt{q} n^{1 / q} m / \varepsilon_{1}^{2} \varepsilon\right)$.

- Step IV: prove $(\mathrm{C} 2)$ for $Y_{k}$. 
Suppose that $Y_{k-1} \stackrel{\text { def }}{=}\left(\sum_{j=0}^{k-2} \frac{\alpha \widehat{L}_{e_{j}}}{\operatorname{Dot}\left(\widehat{L}_{e_{j}}, Y_{j}\right)^{1 / q}}-b^{Y} \cdot I\right)^{-q}$. Since $\operatorname{Tr} Y_{k-1} \leq 1+\varepsilon_{1} \leq 3 / 2$, we must have

$$
Y_{k-1}^{-1 / q}=\sum_{j=0}^{k-2} \frac{\alpha \widehat{L}_{e_{j}}}{\operatorname{Dot}\left(\widehat{L}_{e_{j}}, Y_{j}\right)^{1 / q}}-b^{Y} \cdot I \succeq \frac{2}{3} I .
$$

It is clear from now that it suffices for us to search for $c^{Y} \geq b^{Y}$, because if one selects $c^{Y}=b^{Y}$, he will get $\operatorname{Tr} Y_{k} \leq \operatorname{Tr} Y_{k-1} \leq 1+\varepsilon_{1}$, and therefore $c^{Y}=\bar{b}^{Y}$ is a good lower bound. However, unlike Step III, one cannot perform a simple binary search on $c^{Y}$ because there is no good upper bound for $c^{Y}{ }^{12}$

Instead, consider the following increment-and-binary-search algorithm. Beginning from $b^{Y}$, we first choose $c^{Y}=b^{Y}+\frac{1}{6}$. This choice of $c^{Y}$ ensures that, according to (G.2),

$$
Y_{k}^{-1 / q}=\sum_{j=0}^{k-1} \frac{\alpha \widehat{L}_{e_{j}}}{\operatorname{Dot}\left(\widehat{L}_{e_{j}}, Y_{j}\right)^{1 / q}}-c^{Y} \cdot I \succeq \frac{1}{2} I .
$$

Therefore, we can compute $\operatorname{Tr}\left(Y_{k}\right)=\sum_{e} Y_{k} \bullet \widehat{L}_{e}$ approximately using Lemma G.4. If the approximation computation from Lemma G.4 tells us that $\operatorname{Tr}\left(Y_{k}\right) \geq 1$, we stop the increment of $c^{Y}$. Otherwise, we conclude that $\operatorname{Tr}\left(Y_{k}\right)$ is still less than or equal to $1+\varepsilon_{1}$, and continue to try $c^{Y}=b^{T}+\frac{i}{6}$ for $i=2,3,4, \ldots$. We stop this increment until we find some integer $i$ so that $\operatorname{Tr}\left(Y_{k}\right) \geq 1$.

At this moment, we have that

$$
\begin{gathered}
\operatorname{Tr}\left(\sum_{j=0}^{k-1} \frac{\alpha \widehat{L}_{e_{j}}}{\operatorname{Dot}\left(\widehat{L}_{e_{j}}, Y_{j}\right)^{1 / q}}-\left(b^{Y}+\frac{i-1}{6}\right) \cdot I\right)^{-q} \leq 1+\varepsilon_{1} \quad \text { and } \\
\operatorname{Tr}\left(\sum_{j=0}^{k-1} \frac{\alpha \widehat{L}_{e_{j}}}{\operatorname{Dot}\left(\widehat{L}_{e_{j}}, Y_{j}\right)^{1 / q}}-\left(b^{Y}+\frac{i}{6}\right) \cdot I\right)^{-q} \geq 1 .
\end{gathered}
$$

Therefore, we can perform a binary search for $c^{Y}$ between $b^{Y}+\frac{i-1}{6}$ and $b^{Y}+\frac{i}{6}$ for, and in $\widetilde{O}(1)$ time we can find some value in this interval which satisfies $\operatorname{Tr}\left(Y_{k}\right) \in\left[1,1+\varepsilon_{1}\right]$.

Again, since we always have $c^{Y} \leq O\left(n^{1 / q} / \sqrt{q} \varepsilon\right)$ owing to Lemma G.1. the binary search step costs a running time that is at most $\widetilde{O}\left(c^{Y} q m / \varepsilon_{1}^{2}\right)=\widetilde{O}\left(\sqrt{q} n^{1 / q} m / \varepsilon_{1}^{2} \varepsilon\right)$ owing to Lemma G.4.

The incrementation procedure takes a running time $\widetilde{O}\left(\sqrt{q} n^{1 / q} m / \varepsilon_{1}^{2} \varepsilon\right)$ for each increment of $\frac{1}{6}$. However, throughout the algorithm, we increment $c^{Y}$ by $1 / 6$ at most $O\left(n^{1 / q} / \sqrt{q} \varepsilon\right)$ times in total as per Lemma G.1. This running time, after amortization, is going to be dominated by that of the binary search.

Overall, we have shown that $(\mathrm{C} 2)$ and $(\mathrm{C} 3)$ can be implemented to run in $\widetilde{O}\left(\sqrt{q} n^{1 / q} m / \varepsilon_{1}^{2} \varepsilon\right)$ time (in amortization) per iteration. Since there are a total of at most $\frac{n}{\varepsilon^{2}}$ iterations, the desired running time is obtained.

\section{G.1 Missing Lemmas}

In this subsection, we state and prove Lemma G.3 and Lemma G.4 for the efficient computations of the matrix inverses needed for the previous subsection.

\footnotetext{
${ }^{12}$ In fact, if one is allowed to compute the smallest eigenvalue of $\sum_{j=0}^{k-1} \frac{\alpha \widehat{L}_{e_{j}}}{\operatorname{Dot}\left(\widehat{L}_{e_{j}}, Y_{j}\right)^{1 / q}}$, he can perform a binary search as described in Section 6. However, we have chosen not to implement that algorithm because the running time analysis for the max/min eigenvalue computation is only longer than the current one.
} 
Lemma G.3. Suppose that we are given positive reals $c$ and $s_{0}, \ldots, s_{k-1}$ satisfying $c I-\sum_{j=0}^{k-1} s_{j} \breve{L}_{e_{j}} \nsucceq$ $\frac{1}{2} I$, where each $\breve{L}_{e}$ is the normalized edge Laplacian and $k=O(m)$. Let $q$ be any positive even integer. Then, we can compute a matrix $T \in \mathbb{R}^{m^{\prime} \times m}$ in time $\widetilde{O}\left(c q m / \varepsilon_{1}^{2}\right)$, where $T$ has $m^{\prime}=\Theta\left(\log n / \varepsilon_{1}^{2}\right)$ rows and satisfies that, with probability at least $1-n^{-\Omega(1)}$,

$$
\forall e \in E, \quad X \bullet \breve{L}_{e} \leq\left\|T \chi_{e}\right\|_{2}^{2} \leq\left(1+\varepsilon_{1}\right) X \bullet \breve{L}_{e}, \quad \text { where } X \stackrel{\text { def }}{=}\left(c I-\sum_{j=0}^{k-1} s_{j} \breve{L}_{e_{j}}\right)^{-q} .
$$

Lemma G.4. Suppose we are given positive $s_{0}, \ldots, s_{k-1}$ and a possibly negative real c satisfying that $\sum_{j=0}^{k-1} s_{j} \breve{L}_{e_{j}}-c I \succeq \frac{1}{2} I$, where each $\breve{L}_{e}$ is the normalized edge Laplacian and $k=O(m)$. Let $q$ be any positive even integer. Then, we can compute a matrix $T \in \mathbb{R}^{m^{\prime} \times m}$ in time $\widetilde{O}\left(\mathrm{cqm} / \varepsilon_{1}^{2}\right)$, where $T$ has $m^{\prime}=\Theta\left(\log n / \varepsilon_{1}^{2}\right)$ rows and satisfies that, with probability at least $1-n^{-\Omega(1)}$,

$$
\forall e \in E, \quad Y \bullet \check{L}_{e} \leq\left\|T \chi_{e}\right\|_{2}^{2} \leq\left(1+\varepsilon_{1}\right) Y \bullet \breve{L}_{e}, \quad \text { where } Y \stackrel{\text { def }}{=}\left(\sum_{j=0}^{k-1} s_{j} \check{L}_{e_{j}}-c I\right)^{-q} .
$$

Our proofs to the above lemmas rely on the following auxiliary tools.

\section{G.1.1 Auxiliary Tools}

The first one is the famous Laplacian linear system solver, written in the matrix language.

Theorem G.5. For parameter $\alpha \in[0,1]$. Given any Laplacian matrix $L$ that corresponds to a graph with $m$ edges, there exist an approximation $\bar{L}^{-1}$ which satisfies that, with probability at least $1-n^{-\Omega(1)},(1-\delta) L^{-1} \preceq \bar{L}^{-1} \preceq(1+\delta) L^{-1}$, and for every vector $v \in \mathbb{R}^{n}, \bar{L}^{-1} v$ can be computed in time $\widetilde{O}(m \log (1 / \delta))$.

Proof. The algorithms presented in [ST04] can be expressed as matrices $\bar{L}^{-1}$ which satisfy that, with high probability, for every $x \in \mathbb{R}^{n}$, the vectors $L^{-1} x$ and $\bar{L}^{-1}$ are close under the so-called $L$ norm, or in symbols, $\left\|\bar{L}^{-1} x-L^{-1} x\right\|_{L}^{2} \leq \delta^{2}\left\|L^{-1} x\right\|_{L}^{2}$. After expanding this out using the definition of the $L$-norm, we have

$$
\begin{array}{lr} 
& x^{T}\left(\bar{L}^{-1}-L^{-1}\right) L\left(\bar{L}^{-1}-L^{-1}\right) x \leq \delta^{2} \cdot x^{T} L^{-1} L L^{-1} x \\
& \left(\bar{L}^{-1}-L^{-1}\right) L\left(\bar{L}^{-1}-L^{-1}\right) \preceq \delta^{2} \cdot L^{-1} \\
\Longrightarrow & \left(L^{1 / 2} \bar{L}^{-1} L^{1 / 2}-I\right)^{2} \preceq \delta^{2} I \\
\Longrightarrow & -\delta I \preceq L^{1 / 2} \bar{L}^{-1} L^{1 / 2}-I \preceq \delta I \\
& (1-\delta) L^{-1} \preceq \bar{L}^{-1} \preceq(1+\delta) L^{-1} .
\end{array}
$$

The running time $\widetilde{O}(m \log (1 / \delta))$ follows from that of [ST04].

The next two lemmas are the classical results on approximating $(I-A)^{-q}$ and $(A-I)^{-q}$ using Taylor expansions.

Lemma G.6. The polynomial $\mathrm{P}(A)=I+A+\cdots+A^{d-1}$ satisfies that for all $0 \preceq A \preceq(1-\delta) I$,

$$
0 \preceq(I-A)^{-1}-\mathrm{P}(A) \preceq(1-\delta)^{d} \cdot(I-A)^{-1} .
$$

As a consequence, for every integer $q \geq 1$,

$$
\left(1-q(1-\delta)^{d}\right) \cdot(I-A)^{-q} \preceq \mathrm{P}^{q}(A) \preceq(I-A)^{-q} .
$$


Proof. We first note that for every $x \in[0,1-\delta]$, we have

$$
0 \leq \frac{1}{1-x}-\left(1+x+\cdots+x^{d-1}\right)=x^{d}+x^{d+1}+\cdots=\frac{x^{d}}{1-x} \leq \frac{(1-\delta)^{d}}{1-x} .
$$

As a consequence, we have that

$$
0 \preceq(I-A)^{-1}-\left(1+A+\cdots+A^{d-1}\right) \preceq(1-\delta)^{d} \cdot(I-A)^{-1},
$$

which can be proved by first assuming (without loss of generality) that $A$ is diagonal, and then analyzing each diagonal entry using (G.3).

To prove the result for $(I-A)^{-q}$, we first notice that $(I-A)^{-1}$ and $\mathrm{P}(A)$ are commutable. Therefore, $\mathrm{P}(A) \preceq(I-A)^{-1}$ directly implies $\mathrm{P}^{q}(A) \preceq(I-A)^{-q}$, which gives one side of the inequality. To see the other side, we rewrite

$$
\left(1-(1-\delta)^{d}\right) \cdot(I-A)^{-1} \preceq \mathrm{P}(A)
$$

and then take the $q$-th power on both sides. This yields

$$
\left(1-q(1-\delta)^{d}\right) \cdot(I-A)^{-q} \preceq\left(1-(1-\delta)^{d}\right)^{q} \cdot(I-A)^{-q} \preceq \mathrm{P}^{q}(A)
$$

which finishes the proof of the lemma.

Lemma G.7. The polynomial $\mathrm{P}(A)=A+A^{2}+\cdots+A^{d}$ satisfies that for all $(1+\delta) I \preceq A$,

$$
0 \preceq(A-I)^{-1}-\mathrm{P}\left(A^{-1}\right) \preceq(1+\delta)^{-d} \cdot(A-I)^{-1} .
$$

As a consequence, for every integer $q \geq 1$,

$$
\left(1-q(1+\delta)^{-d}\right) \cdot(A-I)^{-q} \preceq \mathrm{P}^{q}\left(A^{-1}\right) \preceq(A-I)^{-q} .
$$

Proof. We first note that for every $x \geq 1+\delta$, we have

$$
0 \leq \frac{1}{x-1}-\left(x^{-1}+x^{-2}+\cdots+x^{-d}\right)=x^{-d-1}+x^{-d-2}+\cdots=\frac{1}{x^{d}} \frac{1}{x-1} \leq \frac{1}{(1+\delta)^{d}} \frac{1}{x-1} .
$$

As a consequence, we have that

$$
0 \preceq(A-I)^{-1}-\left(A^{-1}+A^{-2}+\cdots+A^{-d}\right) \preceq(1+\delta)^{-d} \cdot(A-I)^{-1},
$$

which can be proved by first assuming (without loss of generality) that $A$ is diagonal, and then analyzing each diagonal entry using (G.4).

To prove the result for $(A-I)^{-q}$, we first notice that $(A-I)^{-1}$ and $\mathrm{P}\left(A^{-1}\right)$ are commutable. Therefore, $\mathrm{P}\left(A^{-1}\right) \preceq(A-I)^{-1}$ directly implies $\mathrm{P}^{q}\left(A^{-1}\right) \preceq(A-I)^{-q}$, which gives one side of the inequality. To see the other side, we rewrite

$$
\left(1-(1+\delta)^{-d}\right) \cdot(A-I)^{-1} \preceq \mathrm{P}\left(A^{-1}\right),
$$

and then take the $q$-th power on both sides. This yields

$$
\left(1-q(1+\delta)^{-d}\right) \cdot(A-I)^{-q} \preceq\left(1-(1+\delta)^{-d}\right)^{q} \cdot(A-I)^{-q} \preceq \mathrm{P}^{q}\left(A^{-1}\right),
$$

which finishes the proof of the lemma.

\section{G.1.2 Missing Proofs of Lemma G.3 and G.4}

Lemma G.3. Suppose that we are given positive reals $c$ and $s_{0}, \ldots, s_{k-1}$ satisfying $c I-\sum_{j=0}^{k-1} s_{j} \breve{L}_{e_{j}} \succeq$ $\frac{1}{2} I$, where each $\breve{L}_{e}$ is the normalized edge Laplacian and $k=O(m)$. Let $q$ be any positive even integer. Then, we can compute a matrix $T \in \mathbb{R}^{m^{\prime} \times m}$ in time $\widetilde{O}\left(c q m / \varepsilon_{1}^{2}\right)$, where $T$ has $m^{\prime}=\Theta\left(\log n / \varepsilon_{1}^{2}\right)$ rows and satisfies that, with probability at least $1-n^{-\Omega(1)}$,

$$
\forall e \in E, \quad X \bullet \breve{L}_{e} \leq\left\|T \chi_{e}\right\|_{2}^{2} \leq\left(1+\varepsilon_{1}\right) X \bullet \breve{L}_{e}, \quad \text { where } X \stackrel{\text { def }}{=}\left(c I-\sum_{j=0}^{k-1} s_{j} \breve{L}_{e_{j}}\right)^{-q}
$$


Proof. Denoting by $A=\frac{1}{c} \sum_{j=0}^{k-1} s_{j} \breve{L}_{e_{j}}$, we have $0 \preceq A \preceq\left(1-\frac{1}{2 c}\right) I$ by the assumption. Now we apply Lemma G.6. and let $\mathrm{P}(A)$ be the matrix polynomial of degree $d=\Theta\left(c \log \left(q / \varepsilon_{1}\right)\right)$ from Lemma G.6. By the approximation guarantee, we have for every edge $e \in E$,

$$
X \bullet \breve{L}_{e}=\left(c I-\sum_{j=0}^{k-1} s_{j} \breve{L}_{e_{j}}\right)^{-q} \bullet \breve{L}_{e}=\left(1 \pm \frac{\varepsilon_{1}}{10}\right) \cdot c^{-q} \cdot \mathrm{P}^{q}(A) \bullet \breve{L}_{e} \text {. }
$$

Therefore, it suffices for us to compute $\mathrm{P}^{q}(A) \bullet \breve{L}_{e}$ for each possible edge $e$.

Next, let $\bar{L}_{G}^{-1}$ be the approximation of $L_{G}^{-1}$ from Theorem G.5 that satisfies

$$
\left(1-\frac{\varepsilon_{1}}{10 d q}\right) L_{G}^{-1} \preceq{\overline{L_{G}}}^{-1} \preceq\left(1+\frac{\varepsilon_{1}}{10 d q}\right) L_{G}^{-1} .
$$

Denoting by $L_{s} \stackrel{\text { def }}{=} \sum_{j=0}^{k-1} \frac{s_{j}}{c} L_{e_{j}}$, we have $A=L_{G}^{-1 / 2} L_{s} L_{G}^{-1 / 2}$. Accordingly, for every edge $e \in E$,

$$
\begin{aligned}
\mathrm{P}^{q}(A) \bullet \breve{L}_{e} & =\operatorname{Tr}\left(\mathrm{P}^{q}\left(L_{G}^{-1 / 2} L_{s} L_{G}^{-1 / 2}\right) L_{G}^{-1 / 2} L_{e} L_{G}^{-1 / 2}\right) \\
& =\operatorname{Tr}\left(\mathrm{P}^{q}\left(L_{G}^{-1} L_{s}\right) L_{G}^{-1} L_{e}\right) \\
& =\operatorname{Tr}\left(\mathrm{P}^{q / 2}\left(L_{G}^{-1} L_{s}\right) L_{G}^{-1} \mathrm{P}^{q / 2}\left(L_{s} L_{G}^{-1}\right) L_{e}\right) \\
& =\operatorname{Tr}\left(\mathrm{P}^{q / 2}\left(L_{G}^{-1} L_{s}\right) L_{G}^{-1} B^{T} W B^{T} L_{G}^{-1} \mathrm{P}^{q / 2}\left(L_{s} L_{G}^{-1}\right) L_{e}\right) \\
& \stackrel{1}{=}\left(1 \pm \varepsilon_{1} / 10\right) \cdot \operatorname{Tr}\left(\mathrm{P}^{q / 2}\left(\bar{L}_{G}^{-1} L_{s}\right) \overline{L_{G}}-1 B^{T} W B^{T} \bar{L}_{G}^{-1} \mathrm{P}^{q / 2}\left(L_{s} \bar{L}_{G}^{-1}\right) L_{e}\right) \\
& =\left(1 \pm \varepsilon_{1} / 10\right) \cdot w_{e} \cdot \chi_{e}^{T} \mathrm{P}^{q / 2}\left({\overline{L_{G}}}^{-1} L_{s}\right){\overline{L_{G}}}^{-1} B^{T} W B^{T}{\overline{L_{G}}}^{-1} \mathrm{P}^{q / 2}\left(L_{s} \bar{L}_{G}-1\right) \chi_{e} \\
& =\left(1 \pm \varepsilon_{1} / 10\right) \cdot w_{e} \cdot\left\|W^{1 / 2} B^{T} \bar{L}_{G}^{-1} \mathrm{P}^{q / 2}\left(L_{s} \bar{L}_{G}^{-1}\right) \chi_{e}\right\|_{2}^{2} .
\end{aligned}
$$

Above, (1) follows because each ${\overline{L_{G}}}^{-1}$ is a $\left(1 \pm \frac{\varepsilon_{1}}{10 d q}\right)$ approximation to $L_{G}^{-1}$, while we have at most $(d-1) q+2 \leq d q$ copies of $L_{G}^{-1}$ in any sequence of the matrix multiplication on the left hand side of (1).

For this reason, we can preprocess by computing $T^{\prime} \stackrel{\text { def }}{=} Q W^{1 / 2} B^{T}{\overline{L_{G}}}^{-1} \mathrm{P}^{q / 2}\left(L_{s}{\overline{L_{G}}}^{-1}\right) \in \mathbb{R}^{m^{\prime} \times n}$, where $Q \in \mathbb{R}^{m^{\prime} \times m}$ is some Johnson-Lindenstrauss random matrix with $m^{\prime}=\Theta\left(\log n / \varepsilon_{1}^{2}\right)$ rows. This matrix $T^{\prime}$ satisfies that, with probability at least $1-O\left(n^{-\Omega(1)}\right)$,

$$
\forall e \in E, \quad\left\|Q W^{1 / 2} B^{T}{\overline{L_{G}}}^{-1} \mathrm{P}^{q / 2}\left(L_{s}{\overline{L_{G}}}^{-1}\right) \chi_{e}\right\|_{2}^{2}=\left(1 \pm \varepsilon_{1} / 10\right)\left\|T^{\prime} \chi_{e}\right\|_{2}^{2} .
$$

Combining (G.5): (G.6), and (G.7) together, we have

$$
\forall e \in E, \quad X \bullet \breve{L}_{e}=\left(1 \pm \varepsilon_{1} / 3\right) \cdot c^{-q} \cdot w_{e} \cdot\left\|T^{\prime} \chi_{e}\right\|_{2}^{2} .
$$

Defining $T \stackrel{\text { def }}{=}\left(\frac{1}{1-\varepsilon_{1} / 3} \cdot c^{-q} \cdot w_{e}\right)^{1 / 2} \cdot T^{\prime}$, we get the desired inequality in Lemma G.3.

Finally, we emphasize that the above computation of $T$ requires $\widetilde{O}\left(d q \cdot m^{\prime} \cdot m\right)=\widetilde{O}\left(c q m / \varepsilon_{1}^{2}\right)$ time. This is because, each row of $T$ can be computed by left multiplying each row of $Q$ with the matrix $W^{1 / 2} B^{T}{\overline{L_{G}}}^{-1} \mathrm{P}^{q / 2}\left(L_{s}{\overline{L_{G}}}^{-1}\right){ }^{13}$ The running time now follows from (i) we need to compute vector-matrix multiplication $O(d q)$ times, which is the power of the polynomial $\mathrm{P}^{q / 2}(\cdot)$, and (ii)

\footnotetext{
${ }^{13}$ This can be implemented as follows. For any row vector of $Q$, denote it by $u^{T} \in \mathbb{R}^{m}$. We first sequentially compute

- $v^{T} \leftarrow u^{T} W^{1 / 2}$

- $v^{T} \leftarrow v^{T} B^{T}$, and

- $v^{T} \leftarrow v^{T} \overline{L_{G}}-1$.

Now, suppose $\mathrm{P}^{q / 2}\left(\dot{L}_{s} \bar{L}_{G}^{-1}\right)=\sum_{i=0}^{d q / 2} c_{i}\left(L_{s} \bar{L}_{G}^{-1}\right)^{i}$ where each $c_{i}$ is the coefficient of the $i$-th power term. We continue and compute

- $w^{T} \leftarrow \overrightarrow{0}$.
} 
Theorem G.5 implies that for inversion $v^{T}{\overline{L_{G}}}^{-1}$ can be computed in time $\widetilde{O}\left(m \log \left(d q / \varepsilon_{1}\right)\right)$ for any vector $v$.

Lemma G.4. Suppose we are given positive $s_{0}, \ldots, s_{k-1}$ and a possibly negative real c satisfying that $\sum_{j=0}^{k-1} s_{j} \breve{L}_{e_{j}}-c I \succeq \frac{1}{2} I$, where each $\breve{L}_{e}$ is the normalized edge Laplacian and $k=O(m)$. Let $q$ be any positive even integer. Then, we can compute a matrix $T \in \mathbb{R}^{m^{\prime} \times m}$ in time $\widetilde{O}\left(\mathrm{cqm} / \varepsilon_{1}^{2}\right)$, where $T$ has $m^{\prime}=\Theta\left(\log n / \varepsilon_{1}^{2}\right)$ rows and satisfies that, with probability at least $1-n^{-\Omega(1)}$,

$$
\forall e \in E, \quad Y \bullet \breve{L}_{e} \leq\left\|T \chi_{e}\right\|_{2}^{2} \leq\left(1+\varepsilon_{1}\right) Y \bullet \check{L}_{e}, \quad \text { where } Y \stackrel{\text { def }}{=}\left(\sum_{j=0}^{k-1} s_{j} \breve{L}_{e_{j}}-c I\right)^{-q} .
$$

Proof. There are two cases: $c>0$ or $c \leq 0$. We begin with the case when $c>0$.

Denoting by $A=\frac{1}{c} \sum_{j=0}^{k-1} s_{j} \breve{L}_{e_{j}}$, we have $A \succeq\left(1+\frac{1}{2 c}\right) I$ by the assumption. Now we apply Lemma G.7. and let $\mathrm{P}(A)$ be the matrix polynomial of degree $d=\Theta\left(c \log \left(q / \varepsilon_{1}\right)\right)$ from Lemma G.7. By the approximation guarantee, we have for every edge $e \in E$,

$$
Y \bullet \breve{L}_{e}=\left(\sum_{j=0}^{k-1} s_{j} \breve{L}_{e_{j}}-c I\right)^{-q} \bullet \breve{L}_{e}=\left(1 \pm \frac{\varepsilon_{1}}{10}\right) \cdot c^{-q} \cdot \mathrm{P}^{q}\left(A^{-1}\right) \bullet \breve{L}_{e} .
$$

Therefore, it suffices for us to compute $\mathrm{P}^{q}\left(A^{-1}\right) \bullet \breve{L}_{e}$ for each possible edge $e$.

Denoting by $L_{s} \stackrel{\text { def }}{=} \sum_{j=0}^{k-1} \frac{s_{j}}{c} L_{e_{j}}$, we have $A^{-1}=L_{G}^{1 / 2} L_{s}^{-1} L_{G}^{1 / 2}$. Next, let ${\overline{L_{s}}}^{-1}$ and ${\overline{L_{G}}}^{-1}$ respectively be the approximation of $L_{s}^{-1}$ and $L_{G}^{-1}$ from Theorem G.5 that satisfy

Accordingly, for every edge $e \in E$,

$$
\begin{aligned}
& \left(1-\frac{\varepsilon_{1}}{10 d q}\right) L_{s}^{-1} \preceq{\overline{L_{s}}}^{-1} \preceq\left(1+\frac{\varepsilon_{1}}{10 d q}\right) L_{s}^{-1}, \text { and } \\
& \left(1-\frac{\varepsilon_{1}}{10 d q}\right) L_{G}^{-1} \preceq{\overline{L_{G}}}^{-1} \preceq\left(1+\frac{\varepsilon_{1}}{10 d q}\right) L_{G}^{-1} .
\end{aligned}
$$

$$
\begin{aligned}
& \mathrm{P}^{q}\left(A^{-1}\right) \bullet \breve{L}_{e}=\operatorname{Tr}\left(\mathrm{P}^{q}\left(L_{G}^{1 / 2} L_{s}^{-1} L_{G}^{1 / 2}\right) L_{G}^{-1 / 2} L_{e} L_{G}^{-1 / 2}\right) \\
& =\operatorname{Tr}\left(\mathrm{P}^{q}\left(L_{s}^{-1} L_{G}\right) L_{G}^{-1} L_{e}\right) \\
& =\operatorname{Tr}\left(\mathrm{P}^{q / 2}\left(L_{s}^{-1} L_{G}\right) L_{G}^{-1} \mathrm{P}^{q / 2}\left(L_{G} L_{s}^{-1}\right) L_{e}\right) \\
& =\operatorname{Tr}\left(\mathrm{P}^{q / 2}\left(L_{s}^{-1} L_{G}\right) L_{G}^{-1} B^{T} W B^{T} L_{G}^{-1} \mathrm{P}^{q / 2}\left(L_{G} L_{s}^{-1}\right) L_{e}\right) \\
& \stackrel{(1)}{=}\left(1 \pm \varepsilon_{1} / 10\right) \cdot \operatorname{Tr}\left(\mathrm{P}^{q / 2}\left({\overline{L_{s}}}^{-1} L_{G}\right){\overline{L_{G}}}^{-1} B^{T} W B^{T}{\overline{L_{G}}}^{-1} \mathrm{P}^{q / 2}\left(L_{G}{\overline{L_{s}}}^{-1}\right) L_{e}\right) \\
& =\left(1 \pm \varepsilon_{1} / 10\right) \cdot w_{e} \cdot \chi_{e}^{T} \mathrm{P}^{q / 2}\left({\overline{L_{s}}}^{-1} L_{G}\right){\overline{L_{G}}}^{-1} B^{T} W B^{T}{\overline{L_{G}}}^{-1} \mathrm{P}^{q / 2}\left(L_{G}{\overline{L_{s}}}^{-1}\right) \chi_{e} \\
& =\left(1 \pm \varepsilon_{1} / 10\right) \cdot w_{e} \cdot\left\|W^{1 / 2} B^{T} \overline{L_{G}}-1 \mathrm{P}^{q / 2}\left(L_{G}{\overline{L_{s}}}^{-1}\right) \chi_{e}\right\|_{2}^{2}
\end{aligned}
$$

Above, (1) follows because each $\bar{L}_{s}^{-1}$ (resp. ${\overline{L_{G}}}^{-1}$ ) is a $\left(1 \pm \frac{\varepsilon_{1}}{10 d q}\right)$ approximation to $L_{s}^{-1}$ (resp. $L_{G}^{-1}$ ), while we have at most $(d-1) q+2 \leq d q$ copies of $L_{s}^{-1}$ and $L_{G}^{-1}$ in any sequence of the matrix multiplication on the left hand side of (1).

- For $i \leftarrow 0$ to $d q / 2$,

$$
\begin{aligned}
& -w^{T} \leftarrow w^{T}+v^{T} . \\
& -v^{T} \leftarrow v^{T} L_{s} . \\
& -v^{T} \leftarrow v^{T} \bar{L}_{G} .
\end{aligned}
$$

In the end, the value of the row vector $w^{T}$ is precisely the desired $u^{T} W^{1 / 2} B^{T}{\overline{L_{G}}}^{-1} \mathrm{P}^{q / 2}\left(L_{s}{\overline{L_{G}}}^{-1}\right)$. 
For this reason, we can preprocess by computing $T^{\prime} \stackrel{\text { def }}{=} Q W^{1 / 2} B^{T}{\overline{L_{G}}}^{-1} \mathrm{P}^{q / 2}\left(L_{G}{\overline{L_{s}}}^{-1}\right) \in \mathbb{R}^{m^{\prime} \times n}$, where $Q \in \mathbb{R}^{m^{\prime} \times m}$ is some Johnson-Lindenstrauss random matrix with $m^{\prime}=\Theta\left(\log n / \varepsilon_{1}^{2}\right)$ rows. This matrix $T^{\prime}$ satisfies that, with probability at least $1-O\left(n^{-\Omega(1)}\right)$,

$$
\forall e \in E, \quad\left\|Q W^{1 / 2} B^{T}{\overline{L_{G}}}^{-1} \mathrm{P}^{q / 2}\left(L_{G}{\overline{L_{s}}}^{-1}\right) \chi_{e}\right\|_{2}^{2}=\left(1 \pm \varepsilon_{1} / 10\right)\left\|T^{\prime} \chi_{e}\right\|_{2}^{2} .
$$

Combining (G.8). (G.9), and (G.10), we have

$$
\forall e \in E, \quad Y \bullet \check{L}_{e}=\left(1 \pm \varepsilon_{1} / 3\right) \cdot c^{-q} \cdot w_{e} \cdot\left\|T^{\prime} \chi_{e}\right\|_{2}^{2} .
$$

Defining $T \stackrel{\text { def }}{=}\left(\frac{1}{1-\varepsilon_{1} / 3} \cdot c^{-q} \cdot w_{e}\right)^{1 / 2} \cdot T^{\prime}$, we get the desired inequality in Lemma G.4.

Finally, we emphasize that the computation of $T$ requires $\widetilde{O}\left(d q \cdot m^{\prime} \cdot m\right)=\widetilde{O}\left(d q m / \varepsilon_{1}^{2}\right)$ time. This is because, each row of $T$ can be computed by left multiplying each row of $Q$ with the matrix $W^{1 / 2} B^{T} \bar{L}_{G}^{-1} \mathrm{P}^{q / 2}\left(L_{G} \bar{L}_{s}^{-1}\right)$. ${ }^{14}$ The running time now follows from (i) we need to compute vector-matrix multiplication $O(d q)$ times, which is the power of the polynomial $\mathrm{P}^{q / 2}(\cdot)$, and (ii) Theorem G.5 implies the inversions $v^{T}{\overline{L_{G}}}^{-1}$ and $v^{T}{\overline{L_{s}}}^{-1}$ can both be computed in time $\widetilde{O}\left(m \log \left(d q / \varepsilon_{1}\right)\right)$, for any vector $v$.

In the second case, if $c \leq 0$, we can write

$$
Y=\left(\sum_{j=0}^{k-1} s_{j} \breve{L}_{e_{j}}-c I\right)^{-q}=\left(L_{G}^{-1 / 2}\left(L_{s}-c L_{G}\right) L_{G}^{-1 / 2}\right)^{-q} .
$$

Therefore, denoting by $L_{s}^{\prime}=L_{s}-c L_{G}$, which is another graph Laplacian matrix (with positive edge weights), we can write

$$
\begin{aligned}
Y \bullet \breve{L}_{e} & =\operatorname{Tr}\left(\left(L_{G}^{-1 / 2} L_{s}^{\prime} L_{G}^{-1 / 2}\right)^{-q} L_{G}^{-1 / 2} L_{e} L_{G}^{-1 / 2}\right) \\
& =\operatorname{Tr}\left(\left(L_{s}^{\prime-1} L_{G}\right)^{-q / 2} L_{G}^{-1}\left(L_{G} L_{s}^{\prime-1}\right)^{-q / 2} L_{e}\right) \\
& =w_{e} \cdot \chi_{e}^{T}\left(L_{s}^{\prime-1} L_{G}\right)^{-q / 2} L_{G}^{-1} B^{T} W B L_{G}^{-1}\left(L_{G} L_{s}^{\prime-1}\right)^{-q / 2} \chi_{e} \\
& =w_{e} \cdot\left\|W^{1 / 2} B L_{G}^{-1}\left(L_{G} L_{s}^{\prime-1}\right)^{-q / 2} \chi_{e}\right\|_{2}^{2} .
\end{aligned}
$$

It is now clear that similar to the previous case, we can approximately compute $L_{s}^{\prime-1}$ and $L_{G}^{-1}$ using Theorem G.5. and apply the Johnson-Lindenstrauss dimension reduction. We skip the detailed proofs here because it is only a repetition.

\section{H Efficient Implementation for Other Problems}

As we have seen in Appendix G. Lemma G.3 and Lemma G.4 are at the core of our efficient implementation for the graph sparsification problem. For each other possible sparsification problem, as long as these two lemmas can be properly revised, we can also obtain fast running times. Let us illustrate how to obtain such running times for two applications below.

Sparsifying sums of rank-1 matrices. To solve the problem in Theorem 2, it is not hard to verify that Lemma G.3 can be revised as follows:

Suppose that we are given positive reals $c$ and $s_{0}, \ldots, s_{k-1}$ satisfying $c I-\sum_{j=0}^{k-1} s_{j} \widehat{L}_{e_{j}} \succeq \frac{1}{2} I$, where each $\widehat{L}_{e_{j}}=v_{e_{j}} v_{e_{j}}^{T}$ is an explicit $n \times n$ rank-1 matrix and $k=O(m)$. Let $q$ be any positive even integer. Then, we can compute a matrix $T \in \mathbb{R}^{m^{\prime} \times n}$ in time $\widetilde{O}\left(\right.$ cqn $\left.^{2} / \varepsilon_{1}^{2}\right)$, where $T$ has $m^{\prime}=$

\footnotetext{
${ }^{14}$ This can be implemented in a similar manner as discussed in Footnote 13.
} 
$\Theta\left(\log n / \varepsilon_{1}^{2}\right)$ rows and satisfies that, with probability at least $1-n^{-\Omega(1)}$,

$$
\forall e \in E, \quad X \bullet \widehat{L}_{e} \leq\left\|T v_{e}\right\|_{2}^{2} \leq\left(1+\varepsilon_{1}\right) X \bullet \widehat{L}_{e}, \quad \text { where } X \stackrel{\text { def }}{=}\left(c I-\sum_{j=0}^{k-1} s_{j} \widehat{L}_{e_{j}}\right)^{-q} \text {. }
$$

The key idea for proving the above variant of Lemma G.3 is to note that the matrix inequality $c I-\sum_{j=0}^{k-1} s_{j} \widehat{L}_{e_{j}} \succeq \frac{1}{2} I$ implies that the condition number for PSD matrix $M \stackrel{\text { def }}{=} c I-\sum_{j=0}^{k-1} s_{j} \widehat{L}_{e_{j}}$ is at most $O(c)$. Therefore, one can use for instance steepest descent (or even conjugate gradient or Chebyshev method) to compute $M^{-1} v$ in time $O\left(c n^{2}\right)$ for every vector $v \in \mathbb{R}^{n}$. Next, one can apply the similar Johnson-Lindenstrauss dimension reduction as presented in the proof of Lemma G.3.

A similar variant of Lemma G.4 can be proved similarly.

In sum, each iteration of our Appendix $\mathrm{F}$ is dominated by the computational time need to (1) compute the matrix $T \in \mathbb{R}^{m^{\prime} \times n}$, which takes time $\widetilde{O}\left(c q n^{2} / \varepsilon_{1}^{2}\right)=\widetilde{O}\left(\sqrt{q} n^{2+1 / q} / \varepsilon \varepsilon_{1}^{2}\right)$, and (2) compute $T v_{e}$ for all $e \in[m]$, which takes time $O\left(m n / \varepsilon_{1}^{2}\right)$. Taking into account that we have $T=n / \varepsilon^{2}$ such iterations, this is a total running time of

$$
O\left(\frac{\sqrt{q} n^{3+1 / q}}{\varepsilon^{2} \varepsilon_{1}^{2}}+\frac{m n^{2}}{\varepsilon_{1}^{2} \varepsilon^{2}}\right)
$$

Subgraph sparsification. Given a weighted undirected graph $G$ that can be decomposed into edge-disjoint subgraphs, the goal of linear-sized subgraph sparsification is to construct a $(1+O(\varepsilon))$ spectral sparsifier $G^{\prime}$ to $G$, so that $G^{\prime}$ consists only of the reweighted versions of at most $n / \varepsilon^{2}$ given subgraphs.

In symbols, suppose that the edges of some weighted undirected graph $G$ of $n$ vertices and $m^{\prime}$ edges are decomposed into a disjoint union $E=\biguplus_{i=1}^{m} E_{i}$. We are interested in finding scalars $s_{e} \geq 0$ with $\left|\left\{e: s_{e}>0\right\}\right| \leq O\left(n / \varepsilon^{2}\right)$ such that, letting $L \stackrel{\text { def }}{=} \sum_{e=1}^{m} s_{e} \cdot L_{G\left[E_{e}\right]}$, where $L_{E_{e}}$ is the graph Laplacian matrix on the subgraph of $G$ induced by $E_{e}$, we have $L_{G} \preceq L \preceq(1+\varepsilon) L_{G}$.

For this sparsification problem, for each $e \in[m]$, we define $\widehat{L}_{e}=\frac{L_{G}^{-1 / 2} L_{G\left[E_{e}\right]} L_{G}^{-1 / 2}}{w_{e}}$ to be the normalized subgraph Laplacian scaled by $w_{e}$. Here, $w_{e}$ is the scaling parameter which ensures that $\operatorname{Tr} \widehat{L}_{e}$ is between $1-\varepsilon_{1}$ and 1 . (It suffices to compute $L_{G}^{-1} \bullet L_{G\left[E_{e}\right]}$ up to a multiplicative $1+\varepsilon_{1}$ error, and then assign $w_{e} \approx L_{G}^{-1} \bullet L_{G\left[E_{e}\right]}$.)

For this particular problem, we do not even need to revise Lemma G.3 or Lemma G.4. Recall that we only need to compute 'matrix inversions' of the form

$$
\left(c^{X} \cdot I-\sum_{j=0}^{k-1} \frac{\alpha \widehat{L}_{e_{j}}}{\operatorname{Dot}\left(\widehat{L}_{e_{j}}, X_{j}\right)^{1 / q}}\right)^{-q} \bullet \widehat{L}_{e},
$$

while each $\widehat{L}_{e_{j}}$ is now —instead of a single (scaled) edge Laplacian matrix - the summation of a few (scaled) edge Laplacian matrices. This remains to be the same problem Lemma G.3 is trying to implement. The total running time for this subgraph sparsification is therefore

$$
\widetilde{O}\left(\frac{\sqrt{q} n^{1+1 / q} m^{\prime}}{\varepsilon_{1}^{2} \varepsilon^{3}}\right)
$$

\section{References}

[ABL11] Jean-Yves Audibert, Sébastien Bubeck, and Gábor Lugosi. Minimax policies for combinatorial prediction games. Proceedings of COLT 2011, 2011.

$\left[\mathrm{AGM}^{+} 10\right]$ Arash Asadpour, Michel X. Goemans, Aleksander Mdry, Shayan Oveis Gharan, and Amin Saberi. An $O(\log n / \log \log n)$-approximation Algorithm for the Asymmetric Trav- 
eling Salesman Problem. In Proceedings of the Twenty-First Annual ACM-SIAM Symposium on Discrete Algorithms - SODA '10, pages 379-389, 2010.

[AGM14] David G. Anderson, Ming Gu, and Christopher Melgaard. An Efficient Algorithm for Unweighted Spectral Graph Sparsification. ArXiv e-prints, abs/1410.4273, October 2014.

[AHK05] Sanjeev Arora, Elad Hazan, and Satyen Kale. Fast Algorithms for Approximate Semidefinite Programming using the Multiplicative Weights Update Method. In 46th Annual IEEE Symposium on Foundations of Computer Science (FOCS'05), pages 339-348. IEEE, 2005.

[AHK12] Sanjeev Arora, Elad Hazan, and Satyen Kale. The Multiplicative Weights Update Method: a Meta-Algorithm and Applications. Theory of Computing, 8:121-164, 2012.

[AHR12] Jacob D. Abernethy, Elad Hazan, and Alexander Rakhlin. Interior-Point Methods for Full-Information and Bandit Online Learning. IEEE Transactions on Information Theory, 58(7):4164-4175, July 2012. An earlier version of this paper has appeared in COLT'08.

[AK07] Sanjeev Arora and Satyen Kale. A combinatorial, primal-dual approach to semidefinite programs. In Proceedings of the thirty-ninth annual ACM symposium on Theory of computing - STOC 'O7, page 227, New York, New York, USA, 2007. ACM Press.

[AO15] Zeyuan Allen-Zhu and Lorenzo Orecchia. Using optimization to break the epsilon barrier: A faster and simpler width-independent algorithm for solving positive linear programs in parallel. In Proceedings of the 26th ACM-SIAM Symposium on Discrete Algorithms, SODA '15, 2015.

[AR09] Jacob Abernethy and Alexander Rakhlin. Beating the adaptive bandit with high probability. In Proceedings of the 22nd Conference on Learning Theory - COLT' 09, pages 280-289, 2009.

[BC12] Sébastien Bubeck and Nicolo Cesa-Bianchi. Regret analysis of stochastic and nonstochastic multi-armed bandit problems. Foundations and trends in machine learning, 5(1):1-122, 2012.

$\left[\mathrm{BFK}^{+} 11\right]$ Nikhil Bansal, Uriel Feige, Robert Krauthgamer, Konstantin Makarychev, Viswanath Nagarajan, Joseph (Seffi) Naor, and Roy Schwartz. Min-max Graph Partitioning and Small Set Expansion. In 2011 IEEE 52nd Annual Symposium on Foundations of Computer Science, pages 17-26. IEEE, October 2011.

[Bha97] Rajendra Bhatia. Matrix Analysis, volume 169 of Graduate Texts in Mathematics. Springer New York, New York, NY, 1997.

[BK96] András A. Benczúr and David R. Karger. Approximating s-t minimum cuts in $\widetilde{O}\left(n^{2}\right)$ time. In Proceedings of the twenty-eighth annual ACM symposium on Theory of computing STOC '96, pages 47-55, New York, New York, USA, 1996. ACM Press.

[BK02] András A. Benczúr and David R. Karger. Randomized Approximation Schemes for Cuts and Flows in Capacitated Graphs. Technical report, July 2002.

[BMDG05] Arindam Banerjee, Srujana Merugu, Inderjit S. Dhillon, and Joydeep Ghosh. Clustering with bregman divergences. The Journal of Machine Learning Research, 6:1705-1749, 2005. 
[BN13] Aharon Ben-Tal and Arkadi Nemirovski. Lectures on Modern Convex Optimization. Society for Industrial and Applied Mathematics, January 2013.

[BSS14] Joshua Batson, Daniel A. Spielman, and Nikhil Srivastava. Twice-Ramanujan Sparsifiers. SIAM Review, 56(2):315-334, May 2014.

[CHS11] Marcel K. de Carli Silva, Nicholas J. A. Harvey, and Cristiane M. Sato. Sparse Sums of Positive Semidefinite Matrices. Technical report, July 2011.

$\left[\mathrm{CKM}^{+} 11\right]$ Paul Christiano, Jonathan A. Kelner, Aleksander Madry, Daniel A. Spielman, and Shang-Hua Teng. Electrical flows, laplacian systems, and faster approximation of maximum flow in undirected graphs. In Proceedings of the 43rd annual ACM symposium on Theory of computing - STOC '11, page 273, New York, New York, USA, October 2011. ACM Press.

[CL06] Nicolo Cesa-Bianchi and Gabor Lugosi. Prediction, Learning, and Games. Cambridge University Press, Cambridge, 2006.

[CLLM10] Moses Charikar, Tom Leighton, Shi Li, and Ankur Moitra. Vertex sparsifiers and abstract rounding algorithms. In 51th Annual IEEE Symposium on Foundations of Computer Science, FOCS 2010, October 23-26, 2010, Las Vegas, Nevada, USA, pages 265-274, 2010.

[DDK11] Constantinos Daskalakis, Alan Deckelbaum, and Anthony Kim. Near-optimal no-regret algorithms for zero-sum games. In Proceedings of the Twenty-Second Annual ACM-SIAM Symposium on Discrete Algorithms - SODA '11, pages 235-254, 2011.

[FS95] Yoav Freund and Robert E Schapire. A desicion-theoretic generalization of on-line learning and an application to boosting. In Computational learning theory, pages 23-37. Springer, 1995.

[GK07] Naveen Garg and Jochen Könemann. Faster and Simpler Algorithms for Multicommodity Flow and Other Fractional Packing Problems. SIAM Journal on Computing, 37(2):630652, January 2007.

[Haz12] Elad Hazan. The convex optimization approach to regret minimization. In Optimization for machine learning, chapter 10, pages 287-304. MIT press, 2012.

[HKS12] Elad Hazan, Satyen Kale, and Shai Shalev-Shwartz. Near-optimal algorithms for online matrix prediction. In Proceedings of the 25th Annual Conference on Learning Theory COLT '12, pages 38.1-38.13, 2012.

[JJUW11] Rahul Jain, Zhengfeng Ji, Sarvagya Upadhyay, and John Watrous. QIP = PSPACE. Journal of the ACM (JACM), 58(6):30, 2011.

[JL84] William B. Johnson and Joram Lindenstrauss. Extensions of Lipschitz mappings into a Hilbert space. Contemporary Mathematics, 26:189-206, 1984.

[KLOS14] Jonathan A. Kelner, Yin Tat Lee, Lorenzo Orecchia, and Aaron Sidford. An AlmostLinear-Time Algorithm for Approximate Max Flow in Undirected Graphs, and its Multicommodity Generalizations. In Proceedings of the 25th Annual ACM-SIAM Symposium on Discrete Algorithms - SODA '14, number 1 in STOC '14, April 2014. 
[Lie73] Elliott H. Lieb. Convex trace functions and the wigner-yanase-dyson conjecture. Advances in Mathematics, 11(3):267-288, 1973.

[Nao12] Assaf Naor. Sparse quadratic forms and their geometric applications [after Batson, Spielman and Srivastava]. Astérisque, 2012.

[Ore11] Lorenzo Orecchia. Fast Approximation Algorithms for Graph Partitioning using Spectral and Semidefinite-Programming Techniques. PhD thesis, EECS Department, University of California, Berkeley, May 2011.

[OSV12] Lorenzo Orecchia, Sushant Sachdeva, and Nisheeth K. Vishnoi. Approximating the exponential, the lanczos method and an $\widetilde{O}(m)$-time spectral algorithm for balanced separator. In STOC '12. ACM Press, November 2012.

[PS14] Richard Peng and Daniel A. Spielman. An efficient parallel solver for SDD linear systems. In Symposium on Theory of Computing, STOC 2014, New York, NY, USA, May 31 June 03, 2014, pages 333-342, 2014.

[PST95] Serge A. Plotkin, David B. Shmoys, and Éva Tardos. Fast Approximation Algorithms for Fractional Packing and Covering Problems. Mathematics of Operations Research, 20(2):257-301, May 1995.

[Rak09] Alexander Rakhlin. Lecture notes on online learning. Draft, 2009. Available at http://www-stat.wharton.upenn.edu/ rakhlin/courses/stat991/papers/ lecture_notes.pdf.

[Roc96] R. Tyrrell Rockafellar. Convex Analysis (Princeton Landmarks in Mathematics and Physics). Princeton University Press, 1996.

[Sha07] Shai Shalev-Shwartz. Online learning: Theory, algorithms, and applications. PhD thesis, Hebrew University, 2007.

[Sha11] Shai Shalev-Shwartz. Online Learning and Online Convex Optimization. Foundations and Trends in Machine Learning, 4(2):107-194, 2011.

[She09] Jonah Sherman. Breaking the multicommodity flow barrier for $o(\sqrt{\log n})$-approximations to sparsest cut. In Proceedings of the 50th Annual IEEE Symposium on Foundations of Computer Science, FOCS '09, pages 363-372, 2009.

[SS11] Daniel A. Spielman and Nikhil Srivastava. Graph Sparsification by Effective Resistances. SIAM Journal on Computing, 40(6):1913-1926, January 2011.

[ST04] Daniel A. Spielman and Shang-Hua Teng. Nearly-linear time algorithms for graph partitioning, graph sparsification, and solving linear systems. In Proceedings of the thirty-sixth annual ACM symposium on Theory of computing - STOC '04, page 81, New York, New York, USA, 2004. ACM Press.

[ST11] Daniel A. Spielman and Shang-Hua Teng. Spectral Sparsification of Graphs. SIAM Journal on Computing, 40(4):981-1025, January 2011.

[Ste10] David Steurer. Fast SDP algorithms for constraint satisfaction problems. In Proceedings of the Twenty-First Annual ACM-SIAM Symposium on Discrete Algorithms - SODA '10, pages $684-697,2010$. 
[Vis14] Nisheeth K. Vishnoi. personal communication, 2014.

[Zin03] Martin Zinkevich. Online convex programming and generalized infinitesimal gradient ascent. In Proceedings of the 20th International Conference on Machine Learning (ICML03), pages $928-936,2003$.

[Zou12] Anastasios Zouzias. A matrix hyperbolic cosine algorithm and applications. In Proceedings of the 39th International Colloquium Conference on Automata, Languages, and Programming - Volume Part I, ICALP'12, pages 846-858, Berlin, Heidelberg, 2012. SpringerVerlag. 